\title{
Plant flavones enrich rhizosphere Oxalobacteraceae to improve maize performance under nitrogen deprivation
}

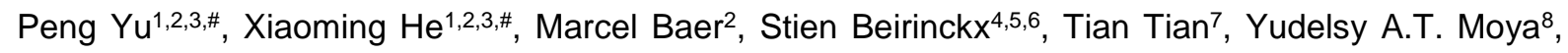
Xuechen Zhang ${ }^{9}$, Marion Deichmann ${ }^{10}$, Felix P. Frey ${ }^{2}$, Verena Bresgen ${ }^{2,3}$, Chunjian Li11, Bahar S. Razavi12, Gabriel Schaaf ${ }^{10}$, Nicolaus von Wirén ${ }^{8}$, Zhen Su', Marcel Bucher ${ }^{13,14}$, Kenichi Tsuda ${ }^{15,16}$, Sofie Goormachtig ${ }^{4,6}$, Xinping Chen ${ }^{1, *}$, Frank Hochholdinger ${ }^{1,2, *}$

${ }^{1}$ College of Resources and Environment, and Academy of Agricultural Sciences, Southwest University (SWU), Chongqing 400715, China

${ }^{2}$ Crop Functional Genomics, Institute of Crop Science and Resource Conservation (INRES), University of Bonn, Bonn 53113, Germany

${ }^{3}$ Emmy Noether Group Root Functional Biology, Institute of Crop Science and Resource Conservation (INRES), University of Bonn, Bonn 53113, Germany

${ }^{4}$ Department of Plant Biotechnology and Bioinformatics, Ghent University, 9052 Gent, Belgium

${ }_{5}^{5}$ Plant Sciences Unit, Flanders Research Institute for Agriculture, Fisheries and Food (ILVO), 9820 Merelbeke, Belgium

${ }^{6}$ Center for Plant Systems Biology, VIB, 9052 Ghent, Belgium

7 State Key Laboratory of Plant Physiology and Biochemistry, College of Biological Sciences, China Agricultural University, Beijing 100193, China

8 Molecular Plant Nutrition, Leibniz Institute of Plant Genetics and Crop Plant Research (IPK), Gatersleben 06466, Germany

9 Department of Biogeochemistry of Agroecosystems, University of Göttingen, Göttingen 37077, Germany

10 Plant Nutrition, Institute of Crop Science and Resource Conservation (INRES), University of Bonn, Bonn 53113, Germany

11 Department of Plant Nutrition, College of Resources and Environmental Science, China Agricultural University, Beijing 100193, China

12 Department of Soil and Plant Microbiome, Institute of Phytopathology, Christian-Albrecht-University of Kiel, Kiel 24118, Germany.

${ }^{13}$ Botanical Institute, Cologne Biocenter, University of Cologne, Cologne 50674, Germany

${ }^{14}$ Cluster of Excellence on Plant Sciences (CEPLAS), University of Cologne, 50674 Cologne, Germany

15 State Key Laboratory of Agricultural Microbiology, College of Plant Science and Technology, Huazhong Agricultural University, Wuhan 430070, China

${ }^{16}$ Department of Plant Microbe Interactions, Max Planck Institute for Plant Breeding Research, Cologne 50829, Germany 

2 During domestication and improvement of plants from natural ecosystems to modern agriculture, root systems rapidly extended their functionality and complexity ${ }^{1,2}$. Root-mediated processes are responsible for major changes in physical and chemical properties of the rhizosphere, which defines the soil volume that is influenced by the properties of the root $^{3}$. It represents a unique soil environment in which complex biogeochemical processes and dynamic biological interactions occur that are mainly driven by plant roots via their secretory activities and soil microorganisms designated the rhizosphere microbiota ${ }^{4}$. Plant-soil-microbe interactions in return affect plant growth, development and health ${ }^{5-8}$. An increasing body of evidence highlights the strong influences of edaphic factors and plant genotype on the assembly and function of the rhizosphere microbiota under controlled conditions ${ }^{9-12}$. Plants are able to shape their rhizosphere microbiota, as supported by the observation that different plant species host specific microbial communities when grown in the same soil ${ }^{13-15}$. Plant root systems display extreme developmental plasticity, enabling them to respond to a wide range of environmental conditions ${ }^{16}$. With respect to the microbiome, plant growth-promoting rhizobacteria affect root growth and development by modulating cell division and differentiation ${ }^{17}$, leading to changes in root system architecture ${ }^{18}$. However, to date, an association between root architectural traits during development and the composition or diversity of defined microbial taxa has not been established yet in crop species. Maize (Zea mays L.) displays a complex root system architecture ${ }^{19}$ and exhibits an exceptional degree of genomic diversity ${ }^{20}$. Hence, maize is an excellent model to explore the relationship between root structure, genetic variation, gene regulation and their interaction with the rhizosphere microbiota. The maize root system comprises different root types that are formed and function at different stages of development ${ }^{19,21}$. Distinct maize root types display specific anatomical characteristics, disparate transcriptomic signatures ${ }^{22}$ and divergent responses to nutrient availability 23,24 . The great architectural and functional diversity of the maize root system has the potential to shape the rhizosphere microbiota composition ${ }^{25}$. Specifically, root type-specific metabolic properties influence microbial communities inhabiting respective root types in maize and rice $26-28$. Conversely, it has been suggested that rootassociated bacteria contribute to differential nitrogen use efficiencies observed in indica and japonica rice varieties ${ }^{29}$, suggesting that root microbiota could alleviate overall nutrient stress in crops. Different maize genotypes display variation in their bacterial microbiome and specific enrichment of different bacterial taxa ${ }^{30,31}$. Among the different maize germplasm groups, sweet corn displays a unique root exudate composition and a tendency to enrich for bacterial taxa that have been associated with nitrogen-fixing activities in the rhizosphere ${ }^{31}$. Maize root exudates contain a variety of metabolites, including substantial amounts of benzoxazinoids and flavonoids that are secreted into the rhizosphere ${ }^{32}$. Benzoxazinoids released by maize roots drive plant performance and defence by shaping rhizosphere microbiota ${ }^{33,34}$. Flavonoids have been considered crucial root-released rhizosphere signal molecules modulating the interaction of roots with microbes ${ }^{35,36}$. Genes such as flavone synthase (FNS) and chalcone synthase colorless2 (C2) are critical for flavonoid biosynthesis in maize ${ }^{37-39}$. Root flavonoids are essential for initiating symbiosis with rhizobia in legumes and also act as auxin transport 
1 developmental processes also in the host plant ${ }^{36}$. Thus, metabolic profiles of specific root types, 2 together with the composition and diversity of microbial taxa in the maize rhizosphere, might be 3 candidate target traits for genetic improvement of nutrient uptake and crop performance under field 4 conditions.

5 Plants strengthen their association with microbes to optimize nutrient acquisition through developmental 6 adaptations of the root system in nutrient poor soil ${ }^{42}$. Nevertheless, the interplay between plants and 7 microbes at the root-soil boundary and its impacts on microbial communities in the rhizosphere and on 8 plant nutrient acquisition have received little attention so far. Therefore, in the present study we 9 elucidated causal interactions between the rhizosphere microbiota, host genetic variation, and host 10 gene expression influencing root developmental processes and nutrient capture under nitrogen 11 deprivation. 
We subjected three longitudinal zones (Fig. 1a) of second whorl crown roots (Supplementary Fig. 1a) of a diverse panel of 20 field-grown maize inbred lines (Supplementary Fig. 1b) in three biological replicates to a transcriptomics experiment and the attached rhizosphere to a fungal and bacterial microbiome analysis. The three root zones include the meristematic zone and the apical part of the elongation zone (zone 1), the basal part of the elongation zone and the apical part of the differentiation zone with root hairs and emerging lateral root primordia (zone 2) and the basal part of differentiation zone with root hairs and emerged lateral roots (zone 3) (Fig. 1a). The separated longitudinal root zones reflect dynamic patterns of root exudation and nutrient uptake capacity, nutrient availability and microbial density along the rhizosphere ${ }^{3}$ (Fig. 1a). A principal component analysis (PCA) together with differential gene analysis illustrated a high transcriptomic similarity between zone 2 and zone 3 in comparison to zone 1 (Fig. 1b; Supplementary Fig. 1c). To confirm functional variation among these zones, differentially expressed genes (Supplementary Dataset 1 ) were functionally classified by Gene Ontology (GO) term enrichment analyses. In the three pairwise comparisons, genes were specifically enriched for root hair elongation (GO:0048767, FDR <0.01) for zone 1 vs zone 2 and zone 1 vs zone 3 and lateral root development (GO:0048527, FDR <0.05) for zone 2 vs zone 3 and zone 1 vs zone 3 (Supplementary Fig. 1c), highlighting that tissue-specific transcriptome changes are in line with the developmental changes of these traits in the three zones.

We further subjected the root transcriptome data to weighted gene co-expression network analyses (WGCNA) (Supplementary Dataset 2) that cluster genes into modules based on their expression profiles and identified 49 co-expression modules, which are groups of highly interconnected genes exhibiting similar gene expression patterns (Supplementary Dataset 3). Specifically, we detected nine modules (M22-M30) displaying specific enrichment along the root development and six co-expression modules (M22-M27) were gradually depleted during root differentiation from the younger meristematic to the older differentiation zone, while three modules (M28-M30) were enriched in zone 3 for all maize genotypes (Supplementary Fig. 2a). Functional annotation of these modules demonstrated enrichment of specific biological processes along the developmental zones (Supplementary Fig. 2b; Supplementary Dataset 4). Among those, growth module 26 is antagonistically associated with defence module 30 (Fig. 1c), highlighting a balancing growth-defence tradeoff during root differentiation. In particular, the auxin signal transduction and the phenylpropanoid biosynthesis pathways were highly interacting within growth module 26 (Supplementary Fig. 3a; Supplementary Dataset 5). For the defence module 30, the maize hub gene ACC1 (acetyl-coenzyme A carboxylase1, Zm00001d004125) was identified as a central player interacting with pathways associated with secondary metabolism and microbial carbon and nitrogen metabolism (Supplementary Fig. 3b; Supplementary Dataset 6). The production of malonyl-CoA through carboxylation of acetyl-CoA by ACC is postulated to be one of the essential substrates for biosynthesis of fatty acids and flavonoids. Moreover, the interconnecting maize genes fab1 (fatty acid biosynthesis1, Zm00001d025201) encoding fatty acid biosynthesis and WHP1 (white 
biosynthesis were identified correspondingly. For nitrogen metabolism, the glutamine synthetase GLN6 (Zm00001d028260) and the asparagine synthetases asn3 (Zm00001d028750) and asn4

3 (Zm00001d047736) were associated with the flow of ammonium into organic nitrogen compounds

4 (Supplementary Dataset 6). Together, these analyses highlight the enrichment of genes associated with carbon/nitrogen metabolism and potential roles of flavonoids on root-rhizosphere interaction along the crown root of maize.

Bacterial community composition in the rhizosphere shifts along the longitudinal root axis while fungal community composition does not

To understand the microbial taxa distribution in the rhizosphere along the root, we analyzed the bacterial and fungal microbiomes by $16 \mathrm{~S}$ rRNA and ITS sequencing (see Methods). Rarefaction analysis demonstrated that sequencing of the microbiome had enough coverage to capture most rhizosphere microbial taxa members for each zone of the 20 maize genotypes (Supplementary Fig. 4a). A PCA showed that, regardless of genotype, a strong shift in bacterial community composition is observed in the maize rhizosphere along the developmental zones (Fig. 1d), but not in fungal microbiome composition (Fig. 1e; Supplementary Table 1). Microbiome richness varied between developmental zones. Zone 3 displayed lower bacterial community diversity than zones 1 and 2, while no significant differences were observed between these zones for fungal community diversity (Supplementary Fig. 4b). Substantial differences in the bacterial community structure of bulk and rhizosphere soils were detected (Supplementary Fig. 5a). Seven of the highly abundant phyla (>96\%) (Supplementary Fig. 4c) significantly differed between bulk and rhizosphere soil (FDR $<0.05$; Supplementary Fig. $5 b)$. Of the rhizosphere bacterial phyla with $>0.1 \%$ abundance, Proteobacteria and Verrucomicrobia were significantly enriched in zone 3 , where lateral root primordia are formed and lateral roots emerge (Supplementary Fig. 5b). In contrast, Acidobacteria, Gemmatimonadetes, Chloroflexi and Nitrospirae were depleted from zones 1 to 3 (Supplementary Fig. 5b). Moreover, Actinobacteria were always present in the rhizosphere of all three root zones (Supplementary Fig. 5b). To investigate root genotypeor/and zone-dependent rhizosphere microbial OTU (operational taxonomic unit) relationships between bacteria and fungi, a co-abundance network was inferred (Supplementary Dataset 7). Consistent with the PCA data, bacterial OTUs showed complex networks while fungal OTUs did not (Supplementary Fig. 6a). Bacterial OTUs related to Nitrospirales showed strong negative correlation with the OTUs corresponding to Verrucomicrobiales (Supplementary Fig. 6b). Furthermore, bacterial OTUs related to Sphingomonadales and Verrucomicrobiales displayed the highest accumulative intra-taxa correlations, although Burkholderiales is the main bacterial taxon interacting between the taxa Sphingomonadales and Verrucomicrobiales (Supplementary Fig. 6c). Thus we hypothesize that changes in composition and diversity of the rhizosphere bacterial microbiome might be associated with transcriptomic divergence along the developmental zones of the root.

Association of the root transcriptome and rhizosphere microbiome along the longitudinal root 
To associate gene expression and microbial OTU abundance, we filtered root RNA-seq reads and microbial amplicon reads for significant correlations of the factors root genotype, zone and genotype by zone interaction between the datasets (Supplementary Dataset 8; Supplementary Table 2). Covariance analysis demonstrated that defined genes expressed in the host roots interact stronger with bacterial OTUs than fungal OTUs (20 times differences, FDR <0.001) in the rhizosphere (Fig. 1f; Supplementary Table 3). To identify how the root zones associate with the rhizosphere microbiota across the genotypes, we correlated eigengenes with the relative abundance of microbial taxa as "trait" and looked for the most significant associations. We searched for significant correlations between these microbial taxa and module eigengene expression values and identified 30 genotype- or zone-specific module-based microbial taxa correlations from phylum to species (Supplementary Dataset 9). We further confirmed that host transcriptome modules had much stronger correlations with bacteria than with fungi at the order level (Fig. 1g; Supplementary Dataset 9). Moreover, growth and defence gene modules had distinct relations with different bacterial orders, e.g. the order Nitrospirales was most positively related to the growth-related modules (Module 22, 25 and 26) and Verrucomicrobiales was negatively related to the defence-related modules (Modules 28-30) (Fig. 1g; Supplementary Dataset 9). Notably, by correlation-based network analyses we demonstrated that root zone-specific gene module expression is strongly associated with specific rhizosphere bacterial taxa along the longitudinal root axis.

\section{Genotype-dependent changes of the root transcriptome and rhizosphere microbiome are} synchronized with plant performance

Next, we asked whether maize growth performance is linked with the genotype-specificity of the interaction of its transcriptomic signature with the rhizosphere microbiome. We investigated 20 phylogenetically distinct genotypes and showed that the genotypes performed differently in the field with respect to biomass production, leaf area, photosynthesis and shoot nitrogen accumulation (Supplementary Fig. 7). Among these genotypes, the inbred line 787 displayed highest leaf area, shoot biomass and nitrogen accumulation in comparison to the other genotypes, while LH93 performed poorly under the same field conditions (Supplementary Fig. 7). By subjecting the root transcriptomes of these genotypes to WGCNA, we identified four modules (M1-M4) associated with the four phylogenetic clades (A-D) and 17 modules corresponding to 17 diverse genotypes (Fig. 2a). However, only few of the genotype-specific modules have significantly enriched GO functions (Fig. 2a), indicating that the genetic dissimilarity is not always linked with functional diversity among genotypes. We therefore hypothesized that genotype-dependent changes in root transcriptomes might underlie the observed variations in rhizosphere microbiome. The maize inbred lines 787 , which showed a unique bacterial community along the longitudinal rhizosphere (Supplementary Fig. 8a) and its corresponding transcriptomic module 5 , displayed the highest correlation with bacterial taxa at the level of phyla and orders compared with all other maize genotypes (Fig. 2b; Supplementary Fig. 8b, c). Moreover, module 5 had a significantly high correlation with the bacterial orders Clostridiales, Bacteroidales, Lactobacillales, Burkholderiales, Chromatiales, Opitutales, Rhodobacterales and Sphingobacteriales in comparison with the other 
those taxa that had been predicted in association with nitrogen metabolism and transformation (e.g., Lachnospiraceae and Nitrosomonadaceae) (Fig. 2c). Interestingly, Oxalobacteraceae are exclusively enriched in the rhizosphere of genotype 787 with the highest correlation with module 5 in comparison to other bacterial families in this study (Fig. 2c; Supplementary Fig. 10). At the genus level, relative abundance of Massilia is significantly enriched in the rhizosphere of inbred line 787 in comparison to the other maize genotypes except for B73 and W64A (Supplementary Dataset 10). In particular, enrichment of Oxalobacteraceae is strongly correlated with growth-related module 26 and module 27 in comparison to the defence-related modules (M28-M30) (Supplementary Dataset 9). Together, these results suggest that specific bacterial taxa become enriched as a consequence of genotype-specific properties and associate with plant nutrition and growth status.

\section{Flavonoid composition coincides with rhizosphere microbiota composition and maize growth properties}

To deepen the understanding of the exceptional performance of inbred line 787, we surveyed the 787 transcriptome for genes with exceptional modular connectivity. Among those, FNSI2 (Zm00001d027423), encoding a flavone synthase displayed the highest modular connectivity within module 5 (Supplementary Dataset 11). Identification of FNSI2 together with the enrichment of the flavonoid biosynthesis-related genes $A C C 1$ and WHP1 in the zone-specific transcriptome analysis highlights the potential importance of flavonoid metabolism and its interaction with rhizosphere microbiota in maize. In line with this observation classical genetic studies demonstrated that root flavonoids are crucial for legume nodule initiation in soybean ${ }^{41}$ and Medicago ${ }^{40}$. In particular, flavones and flavonols play distinct and critical roles in root-microbe interaction during legume-rhizbobium symbiosis $^{43}$. Moreover, the transcriptome data suggested that $F N S I 2$ is specifically expressed in the root cortex. In contrast its paralogue FNSI1 (Zm00001d029744) is predominantly expressed in leaves (Supplementary Fig. 11). Flavone synthases are critical enzymes that catalyze the conversion of the flavanones naringenin and eriodictyol into apigenin and luteolin ${ }^{37}$. Specifically, roots of the inbred line 787 contained and exudated significantly more apigenin and luteolin flavones than inbred line LH93, which accumulated less FNSI2 transcripts (Fig. 2d, e). Furthermore, ${ }^{14} \mathrm{C}$ labelling and imaging experiments (Supplementary Fig. 12a) demonstrated that 787 released significantly higher amounts of root exudates to the rhizosphere than LH93 (Supplementary Fig. 12b and c). We then tested whether the 787-dependent variation in rhizosphere microbiota composition was associated with changes in plant performance and nitrogen use. To this end, sterilization and rhizosphere transplantation trials were performed with the vigorous inbred line 787 and the poorly performing inbred line LH93. The maize inbred line 787 produced more dry shoot biomass than LH93 in nitrogen-poor soil, however growth differences between these two genotypes were not observed when grown in sterilized soil (Fig. 2f). When LH93 was transplanted into soil, in which the inbred line 787 had been grown before (787-grown soil), LH93 significantly increased shoot biomass production. In contrast, the inbred line 787 showed significant growth inhibition when it was transferred to soil, in which the inbred line LH93 was grown before (LH93-grown soil) 18 days after transplantation (DAT) (Fig. 2g). After 28 DAT, shoot biomass of the inbred line 787 in LH93-grown soil and 787-grown soil was not significantly different, whereas LH93 
performed significantly better in 787-grown soil than in LH93-grown soil (Fig. 2g). In particular, the total nitrogen and available mineral nitrogen of the soil prior to transplantation have no significant differences between 787-grown and LH93-grown soils (Supplementary Fig. 13). Thus, we hypothesized that the rhizosphere microbiota conditioned by the vigorous genotype 787 is sufficient to trigger growth of the poorly performing genotype LH93. To further explore the role of the 787-dependent rhizosphere microbiota, we carried out a shotgun metagenomic sequencing of the rhizosphere microbiota of 787 and LH93 (Supplementary Fig. 14a). The metagenomics data validated that the host-specific microbiota of the inbred line 787 differed from that of the inbred line LH93 (Supplementary Fig. 14b). We performed a functional annotation by comparison of quality-filtered reads of these metagenomes to annotated reads using the Clusters of Orthologous Groups (COG) databases (see methods). This revealed that a number of biogenesis, transport and metabolism related processes were significantly enriched in the rhizosphere microbiota associated with genotype 787 in comparison to LH93 (Supplementary Fig. 14c; Supplementary Dataset 12). Overall, our results support the hypothesis that a vigorous plant genotype harbours a rhizosphere with a functionally more diverse microbiome, which results in improved plant performance of an underperforming genotype.

Plant-derived flavones mediate specific variations of rhizosphere bacterial taxa affecting nitrogen capture from the soil

To test if the presence of flavonoids alters rhizosphere-associated microbiota, we compared the performance of $\mathrm{C} 2$ wild type plants with the naturally silenced dominant maize mutant C2-ldf (Colorless2-Inhibitor diffuse), defective in a gene encoding a chalcone synthase (Fig. 3a). C2-Idf mutants displayed highly reduced levels of apigenin-related flavonoids in comparison to $\mathrm{C} 2$ wild type plants $^{38}$. After three weeks of growth in nitrogen-poor and unsterilized soil, C2-Idf plants showed severe chlorosis and necrosis that progressed from older to younger leaves, which is indicative for nitrogen deficiency (Fig. 3b) and growth suppression of the shoot (Fig. 3c). After sterilization of the soil, both genotypes grew similarly weak without differences in biomass production (Fig. 3c). Next, we transplanted C2 wild type and C2-ldf mutant plants to soil, in which these two genotypes were grown before and observed that nitrogen deficiency symptoms of leaves were reduced in C2-Idf mutants planted in C2-grown soil (16 days after transplantation) (Fig. 3d). In contrast, nitrogen deficiency became more severe in $\mathrm{C} 2$ wild type plants that were transplanted into soil in which C2-Idf mutants were grown in comparison to C2 wild types which were replanted into soil in which C2 wild types were grown (20 days after transplantation) (Fig. 3d). These results suggest that the microbiota in soil, in which flavonoid-producing plants were grown, is able to alleviate symptoms of nitrogen deficiency (Fig. $3 \mathrm{~d}, \mathrm{e})$. We therefore propose that flavonoids have triggered the observed promotions in plant growth and nitrogen use by changes of microbial communities. To address this hypothesis, we externally applied different types of flavonoids at different concentrations to a paper roll system and to soil, respectively, and evaluated C2-Idf plant performance. After all, the flavone apigenin at an optimal concentration of $1 \mu \mathrm{M}$ for soil application was determined in the paper roll system (Supplementary Fig. $15 \mathrm{a}$ and $\mathrm{b}$ ). Supplementation of different types of flavonoids at $1 \mu \mathrm{M}$ to $\mathrm{C2}-\mathrm{Idf}$ seedlings had no effects on shoot growth in the paper roll system (Supplementary Fig. 15c) or in sterilized soil (Supplementary Fig. 15d). This suggests that the addition of flavone had no effect on C2-Idf growth in the sterile system. 
Notably, apigenin was the only flavone which restored growth and nitrogen accumulation for C2-Idf plants in unsterilized nitrogen-poor soil in contrast to other flavonoid types (Supplementary Fig. 15d and

3 e). This suggested that the flavone-dependent rhizosphere microbe-driven processes rather than root exudate-derived flavones themselves affected plant growth and nitrogen nutrition. In addition, we examined the bacterial community of $\mathrm{C} 2$ wild type and of the mutant C2-Idf by $16 \mathrm{~S}$ rRNA sequencing and we found that the $\mathrm{C} 2$ wild type rhizosphere recruited an exceptionally high abundance of the taxon Massilia belonging to Oxalobacteraceae from the phylum Proteobacteria (Fig. 3f, g; Supplementary Dataset 13). Sequencing of the bacterial 16S rRNA diversity in the rhizosphere of the C2-Idf mutant supplied with different types of flavonoids in nitrogen poor soil demonstrated that flavonoids reshape the rhizosphere microbiome (Fig. 3h). Moreover, the C2-Idf mutant significantly enriched Massilia at the genus level and Oxalobacteraceae at the family level in apigenin-supplemented soil in comparison to the other flavonoid types (Fig. 3i; Supplementary Dataset 14). Taken together, these results show that plant-derived flavones are likely sufficient to drive the accumulation of specific microbial taxa, affecting plant growth and nitrogen nutritional status from nitrogen-poor soil.

\section{Flavone-conditioned rhizosphere microbiota is sufficient to recover Irt1 performance}

Lateral roots and root hairs are the major components of the root system responsible for water and nutrient uptake along the longitudinal axis of the root. Thus, we hypothesized that lateral root and root hair formation might influence microbiome differentiation along the root. Root phenotyping experiments were performed using maize mutants defective in lateral root and root hair formation under the same nitrogen-poor soil conditions. In brief, the mutants Irt1 and rum1 are lateral root defective. The mutant Irt1 does not form lateral roots on the primary and seminal roots, while rum 1 does not form lateral roots on the primary root and is in addition devoid of seminal roots. The mutant $r$ th 6 is specifically affected in root hair elongation in all root types during the whole growth period. Moreover, rtcs is deficient in the initiation of all seminal and shoot-borne roots while the remaining primary root forms lateral roots and root hairs. Results show that defects in lateral root formation but not in root hair development restricted biomass production in maize shoots (Fig. 4a). Interestingly, the shoot of lateral root mutant Irt1 acquired more nitrogen than that of rum 1 in nitrogen-poor soil, although biomass production severely decreased in both lateral root mutants in comparison to the root hair mutants (Fig. 4b). This observation suggests that Irt1 might have an alternative strategy to acquire nitrogen from nitrogen-poor soil. Moreover, rtcs mutants, which display only a single primary root with lateral roots but no seminal or shoot-borne roots, produced even more shoot biomass and accumulated more nitrogen in the leaves than Irt1 and rum1 (Figs. 4a, 4b). This highlights the contribution of lateral root formation to nitrogen acquisition from nitrogen-poor soil in 4-week-old plants, irrespective of seminal and crown root formation in these genotypes. Furthermore, we sterilized the soil and compared shoot biomass production between wild type, Irt1 and rum 1 with and without microbes. Soil sterilization revealed dramatic repression of biomass production in Irt1, but no decrease was observed in rum1 (Fig. 4c). A flavone profiling experiment using a hydroponic system demonstrated that the Irt1 mutant produces and releases more of the two flavones apigenin and luteolin to the rhizosphere than the respective wild type (Fig. 4d). In contrast, neither 
whether flavone-conditioned rhizosphere microbiota are able to impact growth and development of maize roots, we performed rhizosphere transplantation experiments with the lateral root defective mutants Irt1 and rum1 using nitrogen-poor soil (Fig. 4e). Interestingly, biomass production and nitrogen accumulation of Irt1 in flavone conditioned (787 or C2 wild type grown) soil was promoted in comparison to Irt1 transplanted into Irt1-grown soil, but this stimulation effects of plant performance appeared to be Irt1-specific (Figs. 4e, 4f). Therefore, soil sterilization together with rhizosphere transplantation results highlight important roles of flavone-conditioned microbiota on the growth performance and nitrogen uptake of the lateral root defective Irt1 mutant plants.

\section{Effects of Oxalobacteraceae isolates on lateral root formation and plant growth depend on LRT1}

To investigate whether genetically determined defects in lateral root and root hair formation result in alternative rhizosphere microbiota, 16S rRNA gene amplicon sequencing was performed to profile the rhizosphere microbiota along the three developmental zones of wild type B73, the lateral root defective mutants rum 1 and Irt1 and the root hair defective mutant rth6 in nitrogen-poor soil (Supplementary Fig. 16a). The mutant $r$ tcs was also introduced to evaluate whether seminal roots also affect the microbiota in comparison to rum 1, which also lacks seminal roots. The lateral root defective mutants rum1 and Irt1 attracted similar numbers (ca. 230 OTUs) of genotype-specific bacterial taxa differing from rth6 (170 OTUs) and rtcs (376 OTUs) (Supplementary Fig. 16b). Moreover, $\beta$-diversity of these mutants demonstrated separate clustering between lateral root defective mutants and root hair or seminal root defective mutants, which suggests that during lateral root formation different microbiomes are recruited in comparison to root hair or seminal root formation (Supplementary Fig. 16c). Considering that root hairs are a key component of rhizosphere formation, it was not surprising that root hair formation was an important factor for maintaining the bacterial diversity in comparison to lateral root formation (Supplementary Fig. 16d). Interestingly, in comparison to the wild type B73, the differences of bacterial a-diversity in the rhizosphere along the different zones is not reduced for the lateral root mutants Irt1 and rum1, but in the root hair mutant rth6 (Fig. 5a). This demonstrated that lateral roots are an important trigger for maintaining the spatial pattern of bacterial diversity in the rhizosphere along the developmental zones of maize crown roots. Closer inspection of specifically enriched bacterial taxa revealed distinct mutant-specific microbiota variations (Fig. 5b) and the taxon Oxalobacteraceae was specifically enriched in Irt1 mutant (Fig. 5b; Supplementary Fig. 16e). Lateral root formation of Irt1 was significantly induced by the flavone-conditioned (C2 or 787 grown) rhizosphere microbiota (Fig. 5c). Specific enrichment of Oxalobacteraceae in Irt1 coincides with the finding with C2 (Fig. 3h) and the vigorous inbred lines 787 (Fig. 2c), supporting the hypothesis that specific microbial taxa might be linked with important functions related to growth promotion and nitrogen uptake in Irt1 mutant.

To assess the functional effects of Oxalobacteraceae isolates on growth promotion, we applied 16 strains belonging to Collimonas, Duganella, Herbaspirillum, Janthinobacterium, Massilia, Pseudoduganella from the Oxalobacteraceae isolated from different soil types to the soil where C2-Idf mutant grew in nitrogen-poor soil (Supplementary Dataset 15). Specifically, $16 S$ reads of $C 2$ and the C2-Idf mutant were mapped to those 16 Oxalobacteraceae isolates, confirming that the rhizosphere of C2 plants was significantly enriched (11 out of 16 strains) in Oxalobacteraceae than its mutant C2-Idf 



\section{Discussion}

Understanding the growth and development of plant roots and their adaptation to specific environmental challenges is a prerequisite for the management of nutrient inputs in sustainable agriculture. Interactions between roots and their rhizosphere microbiota are essential for plant growth and performance 7,8 . It is known that dynamic patterns of the root transcriptome synchronize with distinct microbial taxa colonizing different root types ${ }^{26,27}$. However, numerous scenarios and mechanisms by which microbes modulate plant development are still unknown especially in crops ${ }^{44}$. Our large-scale field-study in maize demonstrated that different longitudinal root zones representing distinct phases of development recruit distinct rhizosphere microbiome communities, which are associated with specific transcriptome signatures during root differentiation (Fig. 1). Synchronization of root transcriptome and rhizosphere microbiome is reflected by dynamic root exudation profiles with different compositions along the root developmental zones ${ }^{3}$. Our data suggest that the developmental status or function of root zones has a considerable impact on the establishment of their rhizosphere microbiome. Indeed, the transport rate of nutrients to the shoot varies between different developmental root zones ${ }^{45,46}$, while in turn, localized edaphic factors strongly affect root hair and lateral root formation to increase the uptake of nutrients from the soil47. Based on the strong impact of nitrogen on root system architecture ${ }^{16,48}$, lateral root proliferation along the parental root of maize may also favour nitrogen foraging in nitrogen-poor soil 49,50 . This suggests that causal interactions of specific root zones with the rhizosphere resulted in co-adaptation of roots and their microbiota during development. Intriguingly, bacterial diversity in the rhizosphere microbiome significantly decreases along the developmental zones of roots as illustrated by the two monogenic maize mutants rum1 and Irt1 with defective lateral root development (Fig. 4; Supplementary Fig. 15). This suggests that lateral roots themselves or their exudation profiles are likely a functional determinant of rhizosphere microbiome composition.

Plants can release up to $20-40 \%$ of their photoassimilate-derived carbon via their roots as exudates ${ }^{51}$. This is critical for shaping the rhizosphere microbiota ${ }^{52}$. Recent findings demonstrated that plant-derived coumarins contribute to the reshaping of the specific microbial composition under iron deficiency in Arabidopsis ${ }^{53,54}$. Specific metabolites such as benzoxazinoids have been demonstrated to confer root type-specific changes of microbial communities in maize ${ }^{28,33}$. Thus, the specific metabolite composition of the host roots can considerably influence the establishment of the rhizosphere microbiota and the enrichment of specific taxa. In the present study, correlation-based network analyses identified the flavone synthase encoding gene FNSI2 as a hub gene, which displays very strong associations with specific bacterial taxa (Fig. 2; Supplementary Dataset 11). Targeted metabolite profiling in root extracts and exudates together with ${ }^{14} \mathrm{C}$ labelling of exudates in the rhizosphere suggest, that flavone biosynthesis and subsequent secretion might trigger the functional interactions between host roots and rhizosphere microbes thus improving plant performance (Fig. 2; Supplementary Fig. 13). Our studies on a specific mutation in the chalcone synthase-encoding gene Colorless2 (C2) ${ }^{38}$ together with rhizosphere transplantation experiments including both inbred lines and mutants demonstrated that flavone biosynthesis might be involved in the predominant enrichment of Oxalobacteraceae in the maize rhizosphere (Figs. 2 and 3). These experiments support the notion that the host genotype considerably influences the assembly of the rhizosphere microbiota through root-derived flavones. In this context, 
secreted flavones stimulate the enrichment of the bacterial taxa Oxalobacteraceae in the maize rhizosphere and thus growth and nitrogen acquisition in nitrogen-poor soil. These findings also suggest that plant genotype-specific tailoring of the rhizosphere microbiota is driven by selection and coadaptation of soil-borne microbes through molecular mechanisms including root exudation ${ }^{6,55}$.

The progressively enhanced relative abundance of Oxalobacteraceae from soil via the rhizosphere to the root suggests its potential association with host plant performance in Arabidopsis, barley and maize ${ }^{9,56,57}$. Integration of metabolite profiling of root extracts and exudates together with rhizosphere microbiome analysis of the maize mutants Irt1 and rum1 defective in lateral root formation, demonstrated a causal role of Oxalobacteraceae in maize growth and nitrogen accumulation (Figs. 4 and 5). A series of inoculation experiments confirmed consistent growth promotion and nitrogen uptake effects on Irt1 mutants by independent soil-derived Oxalobacteraceae isolates belonging to the genus Massilia (Fig. 5). Flavonoid biosynthesis genes were specifically enriched in differentiated root zones with emerged root hairs and lateral roots ${ }^{58}$. In fact, Massilia preferentially colonize the mature parts of the root in comparison to the growing root tip ${ }^{59}$. This highlights that flavonoid biosynthesis-related genes might represent important players impacting root development while interacting with rhizosphere microbes. It has been demonstrated that growth-promoting rhizobacteria are able to modulate primary root development ${ }^{17,60,61}$ but also lateral root formation ${ }^{62}$. Production and signalling of auxin ${ }^{61-63}$ are welldefined mechanisms by which bacteria modulate plant root development in Arabidopsis. Notably, both flavone-enriched rhizosphere microbiota and Oxalobacteraceae strains can only rescue the growth and formation of lateral roots in the mutant Irt 1 but not in the auxin-signalling defective mutant rum 1 of maize (Figs. 4 and 5). However, Oxalobacteraceae strains did not in vivo produce indolic compounds such as IAA, indolepyruvic acid and indoleacetamide or display root auxin induction effects using DR5::GUS reporter lines in Arabidopsis (Supplementary Figs. 17 and 18). These results suggest that in Irt1 a specific microbe-mediated pathway alters the balance between proliferation and differentiation during lateral root development, most likely independent of bacteria- and plant-derived auxin-related processes in maize (Fig. 6). Recent studies highlight that shared molecular networks could have evolved through the interplay between rhizobia-mediated nodulation and lateral root development ${ }^{64-66}$. Together, these findings indicate that an LRT1-mediated molecular component that guides plant developmental processes also coordinates the engagement of host roots with microorganisms under nitrogen deprivation. It is important to point out that we performed metabolite profiling and rhizosphere transplantation only for one pair of genotypes (787 and LH93), which is not representative for all maize genotypes. Further studies are needed to investigate whether the effects of growth promotion and nitrogen uptake derived from Oxalobacteraceae are also observed in other maize genotypes. Nevertheless, our subsequent genetic studies using an independent flavonoid biosynthesis mutant $\mathrm{C2}$ Idf together with root defective mutant Irt1 provided consistent results. Moreover, we will survey how the evolutionary conservation of developmental plasticity is conferred by the rhizosphere microbiota in plants. Future studies need to disentangle the complexity of bacteria-bacteria interaction mediated by host-derived metabolites and how this will influence the architecture and function of the root system under nutrient deprivation. 
1 In summary, our data indicate that developmentally controlled biological activities in maize roots are

2 critical for the beneficial interactions with the rhizosphere microbiota. Under nitrogen deprivation, maize

3 assembles specifically bacteria of the taxon Oxalobacteraceae in the rhizosphere. This in turn facilitates

4 nitrogen capture through modulation of lateral root development. A full understanding of the causal

5 relationships between the rhizosphere microbiota, host gene regulation and genetic variation controlled

6 by root development could pave the way for new approaches towards securing high crop yield in a

7 sustainable agriculture. Based on our results, the re-introduction of alleles coding for beneficial flavones

8 into elite maize lines might improve favourable interactions between the root system and its growth

9 promoting rhizosphere biota in maize. Hence, maximization of beneficial plant-microbe interactions as

10 a selection target is a promising strategy for breeding of high-yielding and nutrient efficient crops. 
Field and phytochamber experimental design and maize genotypes

In spring 2018, 72 maize inbred lines from the US Ames panel${ }^{67}$ were hand planted in a randomized complete block design with three biological replications in the field of the long-term experimental station of China Agricultural University located in Quzhou, China (36.52 N, 115.02 E) (Supplementary Fig. 19a). The cropping system on this field plot is a winter wheat - summer maize rotation each year, which is representative of the typical cropping system on the North China Plain. In this long-term field plot no synthetic fertilizers has been applied in the last 30 years. The basic nutrients of the alluvial loamy soil from the whole plots were measured for the top $30 \mathrm{~cm}$ soil before sowing (Supplementary Table 4). Prior to fertilization and planting of the field experiment, we collected bulk soil samples from the different field plots (Supplementary Fig. 19a) and verified homogeneous distribution of bacterial diversity across the field by bacterial 16S rRNA sequencing (Supplementary Fig. 19b). This enabled us to examine stable patterns of rhizosphere microbiota between genotypes and root zones under the conditions of our field. The field was sufficiently irrigated one month prior to sowing to keep proper soil moisture for maize growth. Before sowing of this field experiment, straw from the previous season was removed

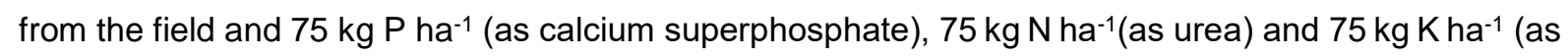
potassium sulfate) were applied and mixed with the surface soil by rotary tillage to meet plant needs for 4-week-old maize. Planting density of all maize inbred lines was approximately 67,500 plants ha-1 with a row width of $60 \mathrm{~cm}$. Precipitation and irrigation, solar radiation and daily average temperature were recorded and no herbicides or pesticides were applied during the growth period (Supplementary Fig. 20).

Of the 72 maize inbred lines, 20 inbred lines, which performed particularly well under the given field conditions were selected for follow-up experiments. These inbred lines represented seven distinct clades (A to G, Supplementary Fig. 1a) of a phylogenetic tree: Clade A (LH38, LH39, 78371A), Clade B (IB014, PHJ75, PHM10, PHN11), Clade C (A632, B73, LH205, PHW51), Clade D (E8501, LH51, LH52, Mo17), Clade E (LH93), Clade F (PHJ31, PHW20) and Clade G (787, W64A).

For the phytochamber pot experiments, the described inbred lines and segregating lines of the monogenic maize root mutants Irt1 (lateralrootless 1), rum1 (rootless with undetectable meristem 1), rth6 (roothairless 6) and rtcs (rootless concerning crown and seminal roots) and their wild type B73 have been pre-germinated for three days in a paper roll system with distilled water and then transferred to soil filled pots $(7 \mathrm{~cm} \times 7 \mathrm{~cm} \times 20 \mathrm{~cm})$ in a 16 -h-light, $26^{\circ} \mathrm{C}$, and 8 -h-dark, $18^{\circ} \mathrm{C}$ cycle. Plants were daily watered to maintain optimal conditions for maize growth. No additional fertilizer has been added during maize growth. The phenotypes of those mutants have been described ${ }^{19}$. Finally, homozygous naturally silenced maize C2-Idf (Colorless2-inhibitor diffuse) mutants and the corresponding $\mathrm{C} 2$ wild type maize seeds were surveyed ${ }^{68}$. The Colorless2 (C2) gene encodes a chalcone synthase, which produces flavones in kernels of pigmented C2 wild type plants ${ }^{38}$.

\section{Determination of aboveground traits in the field}


Aboveground traits were examined for all 20 inbred lines on the day of harvest in the field. The complete aboveground part of the plant was harvested and the dry biomass was determined. Leaf length $(L)$ and width $(W)$ were measured at harvest and leaf area $(A)$ was calculated as $A=L \times W \times K$, where $K=0.75$ was used for fully expanded leaves and $K=0.5$ was introduced for incompletely expanded leaves ${ }^{69}$. Leaves were defined as fully expanded if a ligule was formed. A leaf was considered to have senesced when half or more of its area had yellowed. Chlorophyll content was determined as average of 30 measurements with a SPAD-502 chlorophyll meter (Konica Minolta, Ramsey, NJ, USA) in the middle third of the leaf in longitudinal direction. Dried shoot samples were ground and nitrogen concentrations were determined with a modified Kjeldahl digestion method $^{70}$ from $0.2 \mathrm{~g}$ of plant material.

\section{Sampling of root and rhizosphere soil in the field and phytochamber}

The root and rhizosphere samples were harvested from 28-day-old maize plants in the field. At that time, all nutrients from the seed had been consumed by the seedling growth. Hence, distinct seed sizes do not have any impact on root exudation in different genotypes. Irrigation of the field was terminated 3 days before sample collection to facilitate the rhizosphere harvest. For each genotype, root systems were carefully dug out to a depth of $\sim 40 \mathrm{~cm}$ depth using a spade and all genotypes started to form the $3^{\text {rd }}$ whorl of crown roots and the $2^{\text {nd }}$ whorl of shoot-borne crown roots of a length of ca. $7 \mathrm{~cm}$ were selected (Fig. 1b). Two to three crown roots formed at the $2^{\text {nd }}$ whorl shoot node in all 20 genotypes. We sampled root systems of six random plants from the center of each plot to avoid border effects. Three of the six sampled root systems with clear and integral whorls of crown roots were subjected to root and rhizosphere harvesting. To this end, the whole root system was gently shaken to remove the big soil particles leaving soil attached to the roots defined as the rhizosphere ${ }^{71}$. Subsequently, only the $2^{\text {nd }}$ whorl of the crown roots from all three plants was dissected into three developmentally distinguished zones. Due to the differential availability of root exudates, it has been proposed that the role of rhizosphere microorganisms for nutrient uptake varies along the root $^{3}$. Thus, we separated the longitudinal root zones according to their distinct developmental characteristics, reflecting dynamic root nutrient uptake rate, exudation rate and microbial turnover (Fig. 1a). It should be noted that the boundaries between the different root zones are gradual, thus the separation strategy is mainly based on the emergence of root hairs and visible lateral roots. First, $5 \mathrm{~mm}$ comprising the root cap and the meristematic zone were removed because there was no rhizosphere soil attached in this region of the root. The boundary between elongation zone and differentiation zone was defined by emerging root hairs, which are characteristic for the differentiation zone. Zone 1 was defined as the meristematic zone and apical part of elongation zone comprising the region of $\sim 0.5-2 \mathrm{~cm}$ measured from the tip which did not contain any root hairs for all genotypes. Zone 3 , was defined as the basal part of the differentiation zone and comprised the $\sim 4-7 \mathrm{~cm}$ of the crown roots from the root tip with root hairs and emerged lateral roots. Finally, zone 2 was harvested, which is defined as the remaining segment between zones 1 and 3 covering the basal part of the elongation zone and apical part of differentiation zone with root hair and lateral root primordia formation comprising the region $\sim 2-4 \mathrm{~cm}$ from the root tip. All dissecting steps for root zones have been performed by the same person on ice with sterilized blades in a customized tent on the field site to avoid systematic errors. For each biological replicate per root zone and genotype, 
samples of two to three crown roots of three plants resulting in six to nine crown root segments were pooled. Bulk soil samples across each of the plots were taken in the middle between maize rows at a depth of $30 \mathrm{~cm}$. All root samples were packed in aluminium foil and were immediately frozen in liquid nitrogen and stored at $-80^{\circ} \mathrm{C}$ before extraction of rhizosphere soil. To guarantee high quality root RNA from the field samples, the sampling steps were repeated for additional three plants and the rhizosphere soil was immediately washed away by a Pressure Washer (XM-2081A, Panda Group, Shanghai, China). Subsequently, these roots were separated into three corresponding zones for transcriptome analyses as described above. The pooled zone-specific root samples were dried by clean tissue, wrapped in aluminium foil and were then immediately frozen in liquid nitrogen and stored at $-80^{\circ} \mathrm{C}$ before RNA extraction. For the experiment in the phytochamber, the mutant rhizosphere samples were harvested as described above. Because of lateral root (rum1 and Irt1) and root hair (rth6) defects, the zone-specific segments of the mutants were defined according to the length of their corresponding zones of the wild type seedlings.

\section{Extraction of root zone-specific rhizosphere samples in the lab}

Rhizosphere samples in this study are defined as the combination of the "rhizosphere" and "rhizoplane" compartments ${ }^{71}$. Frozen rhizosphere samples were extracted from the corresponding root segments according to established protocols ${ }^{9}$. In brief, the frozen samples were thawed to room temperature and placed into a $50 \mathrm{ml}$ falcon tube with $20 \mathrm{ml}$ sterile PBS buffer $(\mathrm{pH} 7)$ amended with $0.02 \%$ Silwet L-77 (Sigma). The falcon tubes with samples were shaken for $20 \mathrm{~min}$ with $35 \mathrm{rpm}$ by a cycle agitator (NeoLab). Subsequently, those roots were transferred to a new falcon tube containing $20 \mathrm{ml}$ of PBSSilwet L-77 solution and the shaking step was repeated twice. Afterwards, the rhizosphere samples were centrifuged for $20 \mathrm{~min}$ at $4000 \mathrm{~g}$ at a temperature of $16^{\circ} \mathrm{C}$. This step was repeated twice. Then the supernatant was removed and $300 \mu \mathrm{l}$ of sterile water was added to re-suspend the soil. Finally, the rhizosphere soil (lysing matrix) was transferred into a $1 \mathrm{ml}$ tube and the samples were frozen in liquid nitrogen and stored at $-80^{\circ} \mathrm{C}$ before DNA extraction.

\section{Total RNA isolation from root zone samples}

For transcriptome analysis, the three biological replicates of frozen root samples across the three longitudinal zones were ground in liquid nitrogen. Total RNA was isolated by the RNeasy Plus Universal Mini Kit (Qiagen). RNA quality was assessed by agarose gel electrophoresis and by an Agilent RNA 6000 Nano LabChip in an Agilent 2010 Bioanalyzer (Agilent Technologies). All RNA samples were with high quality as documented by RNA integrity number (RIN) values higher than 6.0. During the quality control steps, an Agilent DNA 1000 LabChip (Agilent Technologies) and an ABI StepOne Plus RealTime PCR System (Applied Biosystems) were used for quantification and quality control of the sample libraries. The cDNA libraries for RNA-seq were constructed with a TruSeq RNA Sample Prep Kit (Illumina). Cluster preparation and PE150 read sequencing were performed according to the Novaseq 6000 guidelines (Illumina).

\section{Transcriptome profiling by RNA-seq and raw sequencing data analysis}



were carried out with Phusion High-Fidelity PCR Master Mix (New England Biolabs) according to the manufacturer's instructions. Subsequently, PCR reactions were mixed with $1 \times$ loading buffer (containing SYB green) and separated on $2 \%$ agarose gels. PCR products with the brightest band between 400-450 bp were chosen for further experiments. PCR products were mixed in equal density ratios and were subsequently purified with the Qiagen Gel Extraction Kit (Qiagen, Germany). Sequencing libraries were generated using the NEBNext巴 Ultra DNA Library Pre Kit for Illumina, following the manufacturer's recommendations and by adding sequence indices. The library quality was assessed on the Qubit ${ }^{@} 2.0$ Fluorometer (Thermo Scientific) and Agilent Bioanalyzer 2100 system. Finally, the library was sequenced on an Illumina HiSeq 2500 platform (Illumina Inc., San Diego, USA) and 250 bp paired-end reads were generated at the Novogene Bioinformatics Technology Co., Ltd. (Beijing, China).

\section{S rRNA gene and ITS1 read data processing}

Paired-end 16S rRNA amplicon sequencing reads were assigned to samples based on their unique barcode and truncated by cutting off the barcode and primer sequence. Paired-end reads were merged using FLASH (v1.2.7) ${ }^{76}$ and the splicing sequences were called raw tags. Quality filtering on the raw tags were performed under specific filtering conditions to obtain the high-quality clean tags according to the QIIME (v1.7.0) quality controlled process ${ }^{77,78}$. Raw tags were compared with the reference Gold database using the UCHIME algorithm (v7) ${ }^{79}$ to detect chimera sequences, which then were removed to obtain the effective tags ${ }^{80}$. Sequence analyses were performed by Uparse software (v7.0.1001) and only sequences with $\geq 97 \%$ similarity were assigned to the same OTUs ${ }^{81}$. For each representative sequence for each OTU, Mothur software was performed against the SSUrRNA database of SILVA Database (release 128$)^{82}$ for species annotation at each taxonomic (kingdom, phylum, class, order, family, genus, species) rank (threshold 0.8-1) ${ }^{83}$. Mitochondria- and chloroplast-assigned OTUs were eliminated. Out of the remaining sequences an OTU table was built (only reads $>2$ copies were retained). In order to study phylogenetic relationships of different OTUs and differences of the dominant species in different samples (groups), multiple sequence alignments were conducted using MUSCLE software (v3.8.31 $)^{84}$. Those sequences that did not align were removed. ITS amplicon data were processed as following: reads were merged, demultiplexed and filtered as described in the previous section and then dereplicated and sorted keeping only those with $>2$ copies. Subsequently, sequences with $\geq 97 \%$ similarity were assigned to the same OTUs using UCLUST (v7). We picked a representative sequence for each OTU and used the UNITE Database ${ }^{85}$ to annotate taxonomic information based on a BLAST algorithm which was calculated by QIIME (v1.7.0). OTU abundance information for bacteria and fungi were normalized using a standard of sequence number corresponding to the sample with the least sequences. Subsequent analysis of $\alpha$-diversity and $\beta$-diversity were all performed based on this output normalized data.

\section{Calculation of $\alpha$ - and $\beta$-diversity of microbiome}

In order to compute $\alpha$-diversity, we rarefied the OTU table and calculated the Shannon's diversity index 
software (v2.15.3). We performed $\beta$-diversity analyses to evaluate differences of samples in species complexity. $\beta$-diversity on both weighted and unweighted unifrac were calculated by QIIME (v1.7.0). We then performed a principal component analysis (PCA), which was applied to reduce the dimension of the original variables using the FactoMineR package and ggplot2 package in R software (v2.15.3) followed by a cluster analysis ${ }^{86}$. A distance matrix of weighted or unweighted unifrac among samples obtained before was transformed to a new set of orthogonal axes, by which the maximum variation factor is demonstrated by first principal component, and the second maximum by the second principal component, and so on. The Unweighted Pair-group Method with Arithmetic Means (UPGMA) clustering was performed as a type of hierarchical clustering method to interpret the distance matrix using average linkage and was conducted by QIIME software (v1.7.0). Tukey's post-hoc ANOVA tests and two-tailed Student's $t$-tests were conducted to determine the microbial diversity of the different zones. The relative abundances of microbial taxa among root zones and genotypes were determined and statistical analyses were based on FDR-corrected Kruskal-Wallis test $(p<0.05)$. Linear discriminant analysis (LDA) effect size (LEfSe) method was applied to differentiate specific bacterial taxa in the rhizosphere of the developmental defective maize mutants. For LEfSe, Kruskal-Wallis and pairwise Wilcoxon tests were performed, followed by LDA to assess the effect size of each differentially abundant taxon ${ }^{87}$. In this study, a $p$-value of $<0.05$ was considered significant for both statistical methods. Bacteria with markedly increased numbers were defined as those with an LDA score $\left(\log _{10}\right)$ of over 2.

\section{Microbial OTU-based correlation network construction}

To evaluate the effects of zone and genotype specificity on microbiome composition, two correlation networks were individually constructed for bacteria and fungi respectively ${ }^{88}$. OTU tables for each dataset were restricted to OTUs comprising $>200$ reads for all 180 samples and produced 5,232 bacterial and 1,439 fungal operational taxonomic units OTUs (Supplementary Dataset 7). For each table, Spearman correlation scores were calculated using the CoNet app (v1.1.1.beta) ${ }^{89}$ for Cytoscape $(v 3.7 .0)^{90}$. Negative edges were discarded, and only edges with correlation scores of $>0.6$ were kept $(p$ $<0.05$, Bonferroni-corrected). To this end, the proportion of inter-taxa and intra-taxa edges (using the edge weights) was calculated for each of the occurring node affiliations. To construct interkingdom cooccurrence networks, the raw read count tables for the $16 \mathrm{~S}$ and ITS datasets were merged to give one table per genotype by zone. OTUs that appeared in less than ten samples were removed. These filtered interkingdom tables were used as an input for SparCC (Python v3.5.2 and numpy v1.11.0) ${ }^{91}$. The analysis was conducted with default parameters and 100 bootstrap samples were used to infer pseudo$p$-values. The inferred correlations were restricted to those having correlations $>0.6$ and $<-0.6(p<0.05$, two-tailed). Within the networks, proportions of inter- and intra-taxa edges were calculated. Intra-taxa refer to edges within bacterial OTUs and fungal OTUs, whereas inter-taxa refer to edges between these groups. The cumulative bacterial or fungal correlation refers to the sum of all intra-taxa correlations for each fungal and bacterial OTU. Visualization of the networks was done with Cytoscape (v3.7.0) as well. 
To fully capture whether our high dimensional data sets of transcriptome and microbiome have the covariance components into genotypes and developmental zones, raw RNA-seq reads for 39,179 protein-coding genes and 180 samples ( $x$, genotypes; $y$, root zones; $z$, replications) were filtered for extremely lowly expressed genes, excluding 4,213 genes with a sum of less than ten reads across all samples thus keeping 34,966 genes. To include only genes and OTUs (bOTUs and fOTUs) in the final analysis, which show variation for one of the factors genotype, zone or genotype-by-zone interaction, we pre-filtered the sets of genes, bOTUs and fOTUs separately. This pre-filtering was done by assessing significance of the three factors, mentioned before, for each gene or OTU, using an analysis of variance ( $R$ function aov () ) with the model:

gene expression genotype + zone + genotype:zone,

OTU abundance genotype + zone + genotype:zone,

where genotype represents the $x$ genotypes, zone represents the $y$ root zones, and genotype:zone the interaction of both effects. We adjusted significance $p$-values using the $R$ function $p$.adjust() with method $=$ "BH" for Benjamini-Hochberg which corresponds to the FDR and adjusts $p$-values according to the number of observations. Genes or OTUs with an FDR $<0.05$ in at least one of the three factors were kept, all other genes or OTUs were filtered out.

In total, 27,092 pre-filtered genes were used as input to identify differentially expressed genes for each OTU (bacterial and fungal) by using the R package DESeq2 (v1.18.1) with the model gene expression $\sim$ OTU abundance. OTU abundance was taken as a trait to explain gene expression. Significant genes were determined with corresponding bOTUs and fOTUs with FDR and $\log _{2}$ fold change for $\alpha$ levels of 0.001. Moreover, we performed the same analysis as above but explaining bOTU abundance with fOTU abundance

bOTU abundance fOTU abundance

and vice versa

fOTU abundance bOTU abundance,

in order to identify direct associations between fungal OTUs and bacterial OTUs.

\section{Network-based integration of host transcriptome and rhizosphere metagenome}

Network-based analysis is the most biologically interpretable tool to analyse the dependency among variables, such as relationships between microbial compositions and gene expressions ${ }^{92}$. The WGCNA is a data-driven method, which discovers the co-clustered gene sets (modules) based on weighted correlations between gene transcripts ${ }^{93}$. To understand how functional changes of host transcriptome associate with the environmental variations of microbial taxa along the longitudinal axis of root, we performed WGCNA to identify the correlations among quantitative data sets using R package: (i) gene co-expression modules among 20 maize lines across three longitudinally distinct zones and (ii) module eigengene value-microbial taxa associations and intra-modular hub genes. WGCNA is designed to be 
genotype-zone specific samples. To robustly construct co-expression networks, only genes with mapped reads $\geq 5$ at least for one genotype with corresponding zones and differentially expressed genes between genotypes/zones generated by CLC Genomics Workbench were kept. The gene expression was normalized according to $\log _{2}(F P K M+1)$ expression. The soft thresholding power $\beta$ calculated adjacencies. To minimize the effects of noise and false associations, we transformed the adjacencies into a topological overlap matrix (TOM), and calculated the corresponding dissimilarities (dissTOM) as 1 - TOM. To hierarchically cluster the genes, we used dissTOM as a distance measure and set the minimum module size (number of genes) to 30 to detect modules. To quantify the coexpression similarities of entire modules, the eigengenes of modules were calculated and clustered based on their correlation and subsequently used to study the crosstalk between functional transcripts and microbial traits ${ }^{94}$. We chose the correlation of 0.9 to cluster the modules. The modules' eigengenes were used to represent the gene expression patterns within a module ${ }^{93}$. Annotation of co-expressed genes within modules was analyzed by AgriGO. The GO terms were further imported to the online tool REVIGO to identify the most connected and represented GO terms ${ }^{95}$. Cytoscape (V3.7.0) will be used to visualize the co-expression network and most connected GO terms.

The relative abundance of the rarefied bacterial and fungal OTU table with taxonomic information was further imported into the WGCNA data frame and each classified taxon was treated as a "physiological trait". The module eigengene was correlated with these microbial traits for the most significant associations. Pearson correlations and their associated $p$-values were then generated for all pairwise comparisons of the module eigengene expression values across the 20 genotypes with three zones. Bonferroni adjustments corrected for multiple comparisons. The modules exhibiting high correlations with microbial taxa were further studied to identify: (i) overrepresented GO categories; and (ii) highly connected module 'hub' genes related to microbial taxa. The highly correlation modules/eigengenes with microbial taxa was visualized by Cytoscape (v3.7.0) as described above.

\section{Shotgun metagenomic analysis}

In addition to taxonomic composition, we performed a shotgun metagenomic sequencing to gain insights into the functional differences between 787 and LH93 rhizosphere microbiomes. A total of three 787 and three LH93 rhizosphere samples were randomly selected from 4-week-old plants in the phytochamber. The isolated DNA integrity and purity was controlled by $1 \%$ agarose gel electrophoresis and NanoPhotometer ${ }^{\circledR}$ spectrophotometer (IMPLEN, CA, USA). DNA concentration was accurately quantified by Qubit ${ }^{\circledR}$ dsDNA Assay Kit in Qubit ${ }^{\circledR}$ 2.0 Flurometer (Life Technologies, CA, USA). For each sample, $1 \mu \mathrm{g}$ of genomic DNA was used with Illumina's TruSeq for library generation using NEBNext® Ultra $^{\text {TM }}$ DNA Library Prep Kit for Illumina (NEB, USA). Briefly, the DNA sample was fragmented by sonication to a size of $350 \mathrm{bp}$, then DNA fragments were end-polished, A-tailed, and ligated with the full-length adaptor for Illumina sequencing with further PCR amplification. At last, PCR products were purified (AMPure XP system) and libraries were analysed for size distribution by Agilent 2100 Bioanalyzer and quantified using real-time PCR. The clustering of the index-coded samples was 
cluster generation, the library preparations were sequenced on an Illumina HiSeq 4000 platform and 150 paired-end reads were generated.

For the bioinformatics steps, raw FASTQ sequences were filter-trimmed to remove the adapter sequences, low quality reads and the host genome sequences using readfq, fqcheck, soap2.21 and bwa-0.7.10. Samples passing quality check were assembled initially using an optimized MEGAHIT (Kmer $=55)$ and mapped to assembled scaftigs using Soap 2.21 and unutilized pair-end reads were collected. Mixed assembly was conducted on the unutilized reads with the same assemble parameter. The scaftigs of each sample and mixed assembled, which were less than $500 \mathrm{bp}$, were trimmed. And the effective scaftigs were used for further analysis and gene prediction. PCA analysis was used for the dimension reduction analysis. Functional annotation was performed by comparison of quality-filtered reads to annotated ones using COG databases ${ }^{96}$ (Release clovr-1.0-RC9).

Flavone quantification in root extracts

Root extracts were prepared from 5-50 mg of lyophilized and ground material using twice $1 \mathrm{ml} 70 \%$ methanol containing $1 \%$ formic acid and once $1 \mathrm{ml} 100 \%$ ethanol-containing $1 \%$ formic acid. After addition of the extraction solvent the reaction tubes were vortexed for $30 \mathrm{~s}$, sonicated for $15 \mathrm{~min}$ in an ultrasonic bath with cooled water and shaken for $2 \mathrm{~h}$ in an overhead shaker at $4{ }^{\circ} \mathrm{C}$. After centrifugation $\left(15,000 \mathrm{rpm}, 30 \mathrm{~min}, 4^{\circ} \mathrm{C}\right)$, the supernatants were transferred and pooled in a $5 \mathrm{ml}$ reaction tube. Extracts were dried in a Speed Vac at $45^{\circ} \mathrm{C}$. Dried samples were re-suspended in 50 $\mu \mathrm{l} 50 \% \mathrm{MeOH}$ containing $0.1 \%$ formic acid. A volume of $40 \mu \mathrm{l}$ was transferred to an UPLC vial and measured values were normalized to total root dry mass. Residual volumes from all samples were pooled, vortexed and transferred to an UPLC vial to be used as quality control sample.

Chromatographic separation was performed on a Vanquish ${ }^{\text {TM }}$ UHPLC system (Thermo Fischer Scientific, San Jose, CA, USA). Flavone (apigenin and luteolin, purity $\geq 99 \%$; Extrasynthese, Genay, France) baseline separation was achieved on a reversed phase Acquity UPLC ${ }^{\circledR}$ HSS T3 column (100 $\AA$, $2.1 \times 150 \mathrm{~mm}, 1.8 \mu \mathrm{m}$, Waters, MA, USA) using a gradient elution of $\mathrm{A}$ (Water, $0.1 \% \mathrm{FA}$ ) and $\mathrm{B}$ (ACN, $0.1 \% \mathrm{FA}$ ) as follows: $0-1 \mathrm{~min}, 10 \% \mathrm{~B} ; 1-2 \mathrm{~min}, 10 \%$ to $30 \% \mathrm{~B} ; 2-5 \mathrm{~min} 30 \%$ to $65 \% \mathrm{~B} ; 5-6 \mathrm{~min}$, $65 \%$ to $85 \% \mathrm{~B}$. Additional steps were included for column rinsing and equilibration yielding a total run time of $13 \mathrm{~min}$. To preserve the integrity of the separation, a guard column (130 $\AA, 2.1 \times 5 \mathrm{~mm}, 1.8 \mu \mathrm{m}$, Waters) was also used. The column temperature was set to $35^{\circ} \mathrm{C}$ and the flow rate to $0.3 \mathrm{ml} / \mathrm{min}$. The injection volume was $5 \mu \mathrm{l}$. The UHPLC system was coupled to Q-Exactive Plus Mass Spectrometer (San Jose, CA, USA) equipped with a HESI source, operating in positive ion mode. Source values were set as follows: Spray voltage $3.5 \mathrm{kV}$; capillary temperature $320^{\circ} \mathrm{C}$; S-lens RF level 50; aux gas heater temp $400^{\circ} \mathrm{C}$; sheath gas flow rate 50; aux gas flow rate 14 . For spectra acquisition a Targeted-SIM/dd-MS ${ }^{2}$ experiment was performed. Resolution in Full Scan was set as 70,000 . For MS/MS experiments resolution 17,500 and NCE $40 \mathrm{~V}$ were used. MS data were acquired and processed by Trace Finder Software (v4.1, Thermo Scientific, San Jose, CA, USA). Ten calibration solutions in the range of 1 to $800 \mathrm{ppb}$ containing apigenin and luteolin standards in $50 \%$ 
curve, the peak area on the extracted ion chromatogram $(\mathrm{XIC})$ of the protonated molecule ion $[\mathrm{M}+\mathrm{H}]^{+}$ was measured. A least-square linear regression weighting by the reciprocal of the concentration was used to best fit linearity of the curve. LOQ was found to be $1 \mathrm{ppb}$. The identification of compounds found in extracts was based on the comparison of retention time, high-resolution $\mathrm{m} / \mathrm{z}$ spectrum and isotope pattern with standards. Additionally, generated $\mathrm{MS}^{2}$ spectra were searched into a custom spectral library for confirmation of the identified compounds.

\section{Flavone quantification in root exudates in hydroponics}

Seven-day-old maize seedlings in the paper roll system were transferred to the hydroponic system for another three weeks of cultivation. For exudate collection, four individual plants for each genotype (787, LH93, B73, Irt1 and rum1) were pooled and rinsed thoroughly three times with sterilized distilled water. Pooled roots were then immersed into a cylinder filled with freshly sterilized water with continuous aeration for 2-h collection. Root exudates were passed through a $0.2 \mu \mathrm{m}$ filter (Filtropur $\mathrm{S}$, Sarstedt) and frozen at $-20^{\circ} \mathrm{C}$. The root fresh weights were recorded in parallel for normalization of the exudates. The identification of apigenin and luteolin was performed as described above. In brief, $10 \mathrm{ml}$ root exudates were first lyophilized and then resuspended in $250 \mu \mathrm{l}$ of $50 \%$ $\mathrm{MeOH}$ containing $0.1 \%$ formic acid. Samples were sonicated for $15 \mathrm{~min}$ and shaken $1 \mathrm{~h}$ in an overhead shaker at $4^{\circ} \mathrm{C}$. After $30 \mathrm{~min}$ centrifugation $\left(15,000 \mathrm{rpm}, 4^{\circ} \mathrm{C}\right), 50 \mu \mathrm{l}$ of the supernatants were subjected to UPLC analysis and measured values were normalized to root fresh weight and root exudate collection period and thus expressed as rate.

\section{Tracking root exudation by ${ }^{14} \mathrm{CO}_{2}$ pulse labelling and ${ }^{14} \mathrm{C}$ imaging in the soil}

To understand how the carbon efflux from the root to the rhizosphere, three-day-old pre-germinated seedlings of 787 and LH93 with equal length of primary roots were transferred and grown in the rhizobox system in a phytochamber $\left(16 \mathrm{~h} / 8 \mathrm{~h} \mathrm{light/dark}\right.$ and $\left.26^{\circ} \mathrm{C} / 18^{\circ} \mathrm{C}\right)$ with six biological replicates. During the three weeks of maize growth, the rhizoboxes were kept inclined at an angle of $45^{\circ}$ in order to make sure that the roots can grow along the lower side of the rhizobox. After 3-week culture, the plants were placed in an airtight chamber with an inner dimension of $1.5 \mathrm{~m} \times 1 \mathrm{~m} \times 1 \mathrm{~m}$ for ${ }^{14} \mathrm{CO}_{2}$ pulse labelling and ${ }^{14} \mathrm{C}$ imaging (Supplementary Fig 12a). The chamber was connected with a glass vial which contained $1 \mathrm{M} \mathrm{NaOH}$ before labeling. $1 \mathrm{M} \mathrm{NaOH}$ in the glass vial was used to trap the $\mathrm{CO}_{2}$ in the chamber for $6 \mathrm{~h}$. After trapping the $\mathrm{CO}_{2}$, a new glass vial contained $\mathrm{Na}_{2}{ }^{14} \mathrm{CO}_{3}$ was connected to the chamber and $1 \mathrm{M} \mathrm{H}_{3} \mathrm{PO}_{4}$ was added to the $\mathrm{Na}_{2}{ }^{14} \mathrm{CO}_{3}$ solution through a pipe. Each plant was labeled with $0.4 \mathrm{MBq}^{14} \mathrm{CO}_{2}$. After $6 \mathrm{~h}$ labeling, the remaining ${ }^{14} \mathrm{CO}_{2}$ was removed by $1 \mathrm{M} \mathrm{NaOH}$ for $1 \mathrm{~h}$.

The ${ }^{14} \mathrm{C}$ distribution pattern was determined along the whole rhizobox by incubating an imaging plate (BAS-MS 2040; 20 by 40 cm; BAS-MS 2040, Fujifilm, Germany) on an open side panel of it for $22 \mathrm{~h}$. The sensitive side of the imaging plate was protected with a plastic bag and was put on the soil surface. The plates were scanned by a ${ }^{14} \mathrm{C}$ imager Typhoon FLA 9500 (Perkin Elmer, Germany) at a spatial resolution of $50 \mu \mathrm{m}$. The digital resolution was 65,536 with 16 bit per pixel (size $0.1 \mathrm{~mm} \times 0.1 \mathrm{~mm}$ ). The ${ }^{14} \mathrm{C}$ signal was calibrated by adding ${ }^{14} \mathrm{C}$ solvent of different radioactivity $(0,100,500,1 \mathrm{MBq})$ to the soil in the wells of a black frame, which was then scanned in the same manner as the samples. 

intensities) at a resolution of $50 \mu \mathrm{m}^{97}$. To quantify the ${ }^{14} \mathrm{C}$ images, the images were converted from a log into a linear system by applying the following equation:

$$
P S L=\left(\frac{R e s}{100}\right)^{2} \times \frac{4000}{S} \times 10^{\left(\frac{Q L}{G}-\frac{1}{2}\right)}
$$

where PSL (photostimulated luminescence) is the quantified value of the image in linear scale, Res is the resolution of the image $(\mu \mathrm{m}$; Res $=50 \mu \mathrm{m})$, $S$ the sensitivity $(S=5,000), L$ the latitude $(L=5)$ and $G$ the gradation $(G=65,535)$.

The vertical distribution of the ${ }^{14} \mathrm{C}$ throughout the soil profile was obtained by subtracting gray values of the frame surrounding the profile as a background. The digital scan of ${ }^{14} \mathrm{C}$ distribution across the vertical soil profile was transformed based on a color map of calibration Finally, quantification of signal intensity was calculated by the total hotspot (\%) per image as area with high ${ }^{14} \mathrm{C}$ activity, then calculated the same for all six replicates and quantified as increment of hotspots (\%).

\section{Maize growth assay by flavonoid compounds in the paper roll system}

To testify how flavonoid compounds affect maize growth, we applied flavone (apigenin and luteolin) and flavonol (quercetin and epigallocatechin) and investigated biomass production of C2-ldf mutant plants which are defective in flavonoid production in the paper roll system and soil pots. For the paper roll experiment, maize kernels were distributed in one line on the Anchor paper (length: $38 \mathrm{~cm}$, width: $25 \mathrm{~cm}$ ) at about $2 \mathrm{~cm}$ from the top with an interspace of 3-4 cm (Supplementary Fig. 14a). Then the paper was rolled to be sure that all kernels stay in place and the rolls with kernels were put into a 5 $\mathrm{L}$ beaker with $1 \mathrm{~L}$ deionized water for culture in the phytochamber $\left(16 \mathrm{~h} / 8 \mathrm{~h} \mathrm{light/dark}\right.$ and $\left.26^{\circ} \mathrm{C} / 18^{\circ} \mathrm{C}\right)$. To avoid inhibitory effects of high concentrations of flavonoids on growth, we first set up a series of induction experiment using different concentrations of apigenin ranging from $1 \mu \mathrm{M}$ to $20 \mu \mathrm{M}$ in the paper roll system. Suitable concentrations of apigenin were further compared with the other three flavonoid compounds to determine if different types of flavonoids have divergent effects on maize growth in the paper roll system. For application of flavonoids in the paper roll system, maize seeds were germinated and primary roots ca. 1-2 cm were selected and treated with different concentrations/types of flavonoids by spraying the solution to the whole area of root growth. Spraying treatment was applied once a day for in total seven days.

Tracking the flavonoid effects on bacterial diversity of the C2-Idf mutant rhizosphere in the pot experiment

To explore how flavonoids interact with soil microbiota, $1 \mu \mathrm{M}$ of the flavonoids determined by induction experiments in the paper roll system was applied into the soil pots. In brief, the same amount of sterilized and unsterilized soil was filled until half of the soil pots and then $20 \mathrm{ml}$ flavonoid solution was spreadwatered into pots. The germinated three-day-old maize plants were transferred to the soil pots with the other half of the sterilized and unsterilized soil with another $20 \mathrm{ml}$ flavonoid solution. The plants were grown in the phytochamber as described before and different types of flavonoid compounds were applied once a week for four weeks in soil pots. The fresh shoot biomass was determined and the $16 \mathrm{~S}$ 
rRNA gene sequencing was performed to quantify the bacterial diversity under the different types of flavonoid types in the C2-Idf mutant rhizosphere as described before.

\section{Plant and soil mineral nutrients determination by elemental analyser and ICP-MS}

Plant samples were heat-treated at $105^{\circ} \mathrm{C}$ for $30 \mathrm{~min}$, dried at $70{ }^{\circ} \mathrm{C}$ to constant weight, weighed and ground into powder. Approx. $6 \mathrm{mg}$ of ground plant material was used to determine the total nitrogen concentration by using Euro Vector CHNS-O Elementalanalyser (HEKAtech GmbH-Analysentechnik, Wegberg, Germany). The data were then calculated into peak areas by the Callidus ${ }^{\mathrm{TM}}$ software providing quantitative results using reference material as a calibrating standard. The same plant samples were used to determine the concentrations of 13 additional mineral nutrients. Leaf material (ca. $10 \mathrm{mg}$ ) was weighed into PTFE digestion tubes and concentrated nitric acid $(0.5 \mathrm{ml} ; 67-69 \%$, Bernd Kraft, Duisburg, Germany) was added to each tube. After $4 \mathrm{~h}$ incubation, samples were digested under pressure using a high-performance microwave reactor (Ultraclave 4; MLS, Leutkirch, Germany). Digested samples were transferred to Greiner centrifuge tubes and diluted with de-ionized (Milli- $\left.{ }^{\circledR}\right)$ water to a final volume of $8 \mathrm{ml}$. Elemental analysis was carried out using inductively coupled plasma mass spectrometry (ICP-MS, Sector Field High Resolution (HR)-ICP-MS, ELEMENT 2, Thermo Fisher Scientific, Dreieich, Germany) with Software version 3.1.2.242. For sample introduction a SC-2 DX Autosampler (ESI, Elemental Scientific, Mainz, Germany) was used. A six point external calibration curve was set from a certified multiple standards solution (Bernd Kraft, Duisburg, Germany). The standard reference material Tomato Leaves (NIST, 1573a) was used to verify data precision and accuracy. The elements Rodium (Rh) and Germanium (Ge) were infused online and used as internal standards for matrix correction.

Fresh soil samples were immediately frozen and stored at $-20^{\circ} \mathrm{C}$ for mineral nitrogen ammonium and nitrate analysis. In parallel, the same portion of fresh soil samples were air-dried and ground into powder for total nitrogen analysis. Determination of nitrogen concentration and data calculation were performed as described for plant samples.

\section{Transplantation of maize rhizosphere in the soil pots}

To investigate the influence of rhizosphere microbiota on plant growth and nutrient acquisition in maize, we examined the growth dynamics and nutritional diagnosis of maize inbred lines (787, LH93) and mutants (C2 wild type and its mutant C2-Idf, B73 wild type and its lateral root mutants Irt1 and rum1) growing with a disturbed microbial community in the rhizosphere under the nitrogen-poor conditions. First, we compared the performance of the same genotype in natural and in sterilized soils and measured the dry biomass after one month. For soil sterilization, we first moistened the soil with sterilized water and autoclaved it in a liquid cycle for $25 \mathrm{~min}$ at $121^{\circ} \mathrm{C}$ and left the sterilized soil at room temperature for at least $24 \mathrm{~h}$. Then we repeated this procedure. After cooling down, the sterilized soil was stored at $4{ }^{\circ} \mathrm{C}$ for stabilization for at least one week and was then used for growing plants. For rhizosphere transplantation, surface-sterilized seeds of maize were germinated in the paper roll system with sterilized water and transferred into pots with natural field soil from Cologne, Germany ${ }^{9}$. For transplantation, the 1-month-old maize plants were carefully removed from the pot, leaving most of the 
rhizosphere soils attached to the roots. This was achieved by gently crushing the soil adhering to the maize roots. The roots were then washed thoroughly in $25 \mathrm{ml}$ of autoclaved deionized water by $5 \mathrm{~min}$ sonication in a water bath (TRANSSONIC T420, Elma, Germany) to remove most of the rhizosphere soil. The removed rhizosphere soil suspension was returned to the original pots in which the uprooted maize plants were grown. The newly germinated seeds with 1-2 cm primary roots were then replanted either into pots with the soils in which the same genotype was grown or into pots with soil in which a different genotype was grown. Plants were monitored for nutritional deficiency symptoms over time after inoculation, and nitrogen deficiency was rated as the ratio of yellowish area from the tip to the total leaf area. The dry biomass of the shoot was also determined after 18 and 28 days of growth in the soil pots.

\section{Growth promotion assay by inoculation of Oxalobacteraceae isolates in the soil pots}

To check whether Oxalobacteraceae can promote maize growth under nitrogen poor conditions, the C2-Idf and another lateral root defective mutants Irt1 and rum 1 were inoculated by independent strains isolated from natural soils from Cologne, Germany ${ }^{9}$ (50.96 N, $\left.6.86 \mathrm{E}\right)$ and Merelbeke, Belgium ${ }^{57}$ (50.58 $\mathrm{N}, 3.46 \mathrm{E}$ ), respectively (Supplementary Dataset 15). Before inoculation of these 16 strains, we first mapped the $16 \mathrm{~S}$ reads for rhizosphere samples of $\mathrm{C} 2$ and the C2-Idf mutant to the sequences of 16 Oxalobacteraceae strains (3 Collimonas strains, 1 Duganella strain, 1 Herbaspirillum strain, 2 Janthinobacterium strains, 4 Massilia strains, 2 Pseudoduganella strains and 3 strains with unknown genera) using HISAT2 (http://daehwankimlab.github.io/hisat2/) with default parameters. Before the inoculation experiment, the maize seeds were surface-sterilized. Briefly, seeds were washed 5 min with sterile water, $2 \mathrm{~min}$ with $70 \%(\mathrm{v} / \mathrm{v})$ ethanol, $20 \mathrm{~min}$ with a bleach solution $(29 \mathrm{ml}$ sterile water, $15 \mathrm{ml}$ $\mathrm{NaClO}, 12-13 \%(\mathrm{v} / \mathrm{v})$ stock solution of $1 \mathrm{ml}$ Tween 20), and finally 5 times for $15 \mathrm{~min}$ with sterile water. Seeds were then dried before pre-germination. The seeds were pre-germinated for $48 \mathrm{~h}$ then seedlings with similar primary root length $(\mathrm{ca} .1-2 \mathrm{~cm}$ ) were selected for inoculation. Oxalobacteraceae strains (6 strains from Belgian collections and 10 strains German collections) were cultured in R2A liquid medium overnight at $28{ }^{\circ} \mathrm{C}$ followed by centrifuged the culture at $1500 \mathrm{rcf}$ for $10 \mathrm{~min}$. Then the pellet was resuspended with $1 \times$ PBS buffer and diluted until $\mathrm{OD}_{600} 0.02$. For each round of inoculation, at least 10 seedlings were inoculated by shaking in a bacterial solution for $3 \mathrm{~h}$ and more than 6 seedlings were transferred to the sterilized natural Cologne soil with low nitrogen and grown in the phytochamber (16 h/8 h light/dark and $26^{\circ} \mathrm{C} / 18^{\circ} \mathrm{C}$ ). Specifically, we also performed the inoculation for Irt1 mutants using the same four Massilia strains to understand their functions under the nitrogen-poor soil with sufficient supply of nitrogen. All pots were randomized on one tray for each biological replicate and each pot was watered with $20 \mathrm{ml}$ sterilized $\mathrm{dH}_{2} \mathrm{O}$ every day. For high nitrogen treatment, $20 \mathrm{ml}$ nitrogen-rich nutrient solution ( $2 \mathrm{mM} \mathrm{NH}_{4} \mathrm{NO}_{3}$ ) was complemented to each pot with the same nitrogen-poor soil before potting and solution was supplied once a week. After four weeks of growth, plants were harvested and the total dry weight was determined. The lateral root density was calculated as the number of emerged lateral roots per unit length $(\mathrm{cm})$ of the main root by hand. The effect of each strain was tested for two rounds of experiments.

\section{Detection of indolic compounds production by Oxalobacteraceae strains}


To check whether Oxalobacteraceae strains can produce indolic compounds, those strains were cultured in R2A medium with or without $0.1 \%$ tryptophan for $24 \mathrm{~h}$ at $28{ }^{\circ} \mathrm{C}$. Analyses were performed on supernatant and bacterial cultures. Standard IAA curves were determined using 0, 0.5, 20, 50 and $100 \mu \mathrm{g} / \mathrm{ml}$ in R2A. The colorimetric assay was performed using Salkowski reagent ${ }^{98}$, which consisted of $4.5 \mathrm{~g}$ of $\mathrm{FeCl}_{3}$ and $22.3 \mathrm{ml}$ of $\mathrm{H}_{2} \mathrm{SO}_{4}$ and $77.7 \mathrm{ml}$ water. $1 \mathrm{ml}$ of Salkowski reagent was mixed with 1 $\mathrm{ml}$ of the sample solution, and the mixture was left in the dark for $30 \mathrm{~min}$ at room temperature. The absorption spectra of a $200 \mu \mathrm{l}$ mixture was determined at $530 \mathrm{~nm}$ in 96 well plates. Three technical replicates were performed for each sample.

\section{DR5::GUS staining of auxin induction by Oxalobacteraceae in Arabidopsis}

To validate whether Oxalobacteraceae strains can induce auxin production, Oxalobacteracea strains were cultured in R2A medium overnight at $28^{\circ} \mathrm{C}$. Bacterial cultures were centrifuged and bacterial cells were resuspended in $1 \times$ PBS buffer. The concentration of the inoculums was adjusted to OD600 = 0.01. The DR5::GUS auxin responsive lines were used to study the auxin response of the bacterial inoculation in Arabidopsis. In brief, DR5::GUS Arabidopsis seeds were surface sterilized and vernalized for two days before sowing on $1 / 2$ MS medium. The roots of 4 -day-old seedlings were inoculated with 8 $\mu \mathrm{l}$ of the bacterial inoculum. GUS staining was performed $3 \mathrm{~h}$ and 3 days after inoculation. GUS signalling was checked under the light microscope (Leica) according to the manufacture instructions. The experiment was performed in three replicates on eight seedlings per replicate.

\section{Data availability}

All raw plant RNA sequencing data, rhizosphere bacterial $16 \mathrm{~S}$ and fungal ITS and shotgun metagenomic sequencing data reported in this paper were deposited in the Sequence Read Archive (http://www.ncbi.n/m.nih.gov/sra) under accession number SRP263360. RNA sequencing reads were mapped to the maize reference genome sequence v4 (https://www.maizegdb.org/genome/genome_assembly/Zm-B73-REFERENCE-GRAMENE-4.0). The SSUrRNA database from SILVA database (release 128, 2016, https://www.arb-silva.de/) and UNITE database (v7.2, 2017, https://unite.ut.ee/) were used for analysing the bacterial $16 \mathrm{~S}$ and fungal ITS sequences, respectively. The AgriGO (v2.0, 2017, http://systemsbiology.cau.edu.cn/agriGOv2/) and REVIGO databases (2017, http://revigo.irb.hr/) were used for functional GO analysis of maize genes. Protein-protein interaction networks of enriched gene modules were generated by STRING database (v10.5, https://version-10-5.string-db.org/). Functional annotation of shotgun metagenomic sequencing was performed using COG databases (Release clovr-1.0-RC9). We deposited customized scripts about association of gene modules with microbial taxonomic traits in the following GitHub repository: https://github.com/PengYuMaize/Yu2021NaturePlants. All statistical source data are provided with this paper. 


\section{Figure 1. Interkingdom multi-omics demonstrates causal plant-microbial interactions in the} rhizosphere during root development. a, Schematic illustration of plant-microbe interactions along the developmental zones and illustration of the separated root zones. Zone 1, the meristematic zone and the apical part of elongation zone; Zone 2, basal part of the elongation zone and apical part of differentiation zone with root hairs and lateral root primordia; Zone 3, the basal part of the differentiation zone with root hairs and emerged lateral roots. MZ, meristematic zone; EZ, elongation zone; DZ, differentiation zone. Dynamic patterns of exudation rate, microbial density, root nutrient uptake capacity and soil nutrient availability along the rhizosphere are modified from Marschner (2012) ${ }^{3}$. Principal component analysis (PCA) displaying the transcriptomic shift (b) and the dissimilarity of bacterial (d) and fungal (e) diversity along the root developmental zones $(p=0.001$, permutational multivariate analysis of variance by ANOSIM). c, Network analysis of significantly enriched GO terms among the nine developmental zone-associated modules. The proportional distribution of $\mathrm{GO}$ terms is visualized by REVIGO and only the major host GO term representing the highest proportion is indicated by different colour codes corresponding to Supplemental Dataset 4. The whole panel of representative GO terms identified by TreeMap view of REVIGO (\%) is provided in the Supplementary Dataset 4 . The node size corresponds to the total number of interacted GO terms for each module. The thickness of the edges denotes the number of overlapped GO terms between each two modules. The solid and dotted edges indicate positive and negative relationships, respectively. Growth and defence related modules are highlighted with blue and red outlines, respectively. f, Covariance analysis highlighting direct associations between host root expressed genes and rhizosphere microbial OTUs (FDR corrected $p<0.001)$. g, Functional associations between gene-enriched modules and microbial orders. Growth and defence related modules are highlighted with blue and red outlines, respectively. The size of the circle corresponds to the sample size. The cumulative correlation is calculated as the sum of Pearson's correlations between modules and their corresponding microbial orders. $p$ values, which were calculated using two-tailed Wilcoxon rank sum test, are indicating significance. The boxes span from the first to the third quartiles, the centre lines represent the median values and the whiskers show the data that lie within the 1.5 interquartile range of the lower and upper quartiles. The data points at the ends of the whiskers represent the outliers.

Figure 2. Host transcriptome and rhizosphere microbiome interactions affecting maize performance. a, Identification of phylogenetic and genotype specific gene modules by WGCNA network analyses. Each module is functionally annotated by gene ontology (GO) analysis and statistical test by Fisher's exact test with Yekutieli adjustment (FDR corrected $p<0.05$ ). Coloured dots and bars correspond to different inbred lines and their phylogenetic relationships in the field experiment (Supplementary Figure 1a). b, Correlation-based network between genotype-specific modules (nodes) and the bacterial taxa (triangles) at the phylum level. Pearson's correlation is calculated by relating the eigengene of each module with the relative abundance of each taxon using WGCNA "trait" function. Heat map indicates FDR corrected $p$ values $<0.05$. The node size corresponds to the size of each module. The thickness and the colour of the edges denotes the strength and the significance of 
correlations. The solid and dotted edges indicate positive and negative correlations, respectively. c, Genotype 787-specific module-taxa correlation and relative abundance (RA) at the family level. The plot shows bacterial families with $\geq 1 \%$ relative abundance. Average relative abundance values are indicated by circle size. Coloured circles denote Pearson's correlation coefficient (PCC) of each family with eigengene of module 5. d, Transcript abundance of module eigengene corresponding to genotype 787 in comparison to the other genotypes. FNSI2, flavone synthase typel2. $n=9$ biologically independent samples. Significances were indicated between inbred line 787 and other genotypes by exact $p$ values (ANOVA, Tukey HSD). e, Concentrations of the two flavones apigenin and luteolin in root extracts or exudates. For root extracts and exudates, $n=4$ and $n=5$ biologically independent samples were collected, respectively. Data are the mean \pm s.e.m. For each flavone type, $p$ values were calculated using two-tailed unpaired t-tests according to the F-test results. $\mathbf{f}$, Growth performance of 787 and LH93 grown in sterilized and unsterilized nitrogen-poor soil. $p$ values were calculated using two-tailed unpaired t-tests according to the F-test results and were adjusted for each soil condition. $\mathbf{g}$, Recovery of LH93 growth with 787-conditioned soil by rhizosphere transplantation. Significances were indicated among transplanted groups by exact $p$ values (ANOVA, Tukey HSD). For $\mathbf{f}$ and $\mathbf{g}, n=10$ biologically independent samples over two independent experiments. DAT, days after transfer. For $\mathbf{d}$, $\mathbf{f}$, and $\mathbf{g}$, the boxes span from the first to the third quartiles, the centre lines represent the median values and the whiskers show the data that lie within the 1.5 interquartile range of the lower and upper quartiles. The data points at the ends of the whiskers represent the outliers.

Figure 3. Changes in rhizosphere microbiota conditioned by plant-derived flavones promote maize growth and nitrogen uptake in nitrogen-poor soil. a, Simplified flavone biosynthesis pathway. The enzymes in the pathway are: $\mathrm{ACC}$, acetyl-CoA carboxylase; $\mathrm{CHI}$, chalcone isomerase; $\mathrm{CHS}$, chalcone synthase; F3H, flavanone 3-hydroxylase; FLS, flavonol synthase; FNS, flavone synthase. Mutation of $\mathrm{CHS}$ in the C2-Idf mutant is highlighted in red. $\mathbf{b}$, Nitrogen deficient phenotype in leaves of the maize mutant C2-Idf compared to wild-type C2 leaves. c, Growth performance of 3-week-old C2 wild-type and C2-Idf mutant plants grown in nitrogen-poor soil with and without prior sterilization. $n=10$ biologically independent samples over two independent experiments. For each condition, $p$ values were calculated using two-tailed unpaired t-tests according to the F-test results. The boxes span from the first to the third quartiles, the centre lines represent the median values and the whiskers show the data that lie within the 1.5 interquartile range of the lower and upper quartiles. The data points at the ends of the whiskers represent the outliers. $\mathbf{d}$, Soil in which C2 plants were grown rescues the nitrogen deficient phenotype of C2-Idf mutants by rhizosphere transplantation. Each data point represents the mean nitrogen deficiency index (mean \pm s.e.m.). $n=5$ biologically independent samples. Different letters indicate significantly different groups ( $\mathrm{BH}$ adjusted $p<0.05$, Wilcoxon rank sum test). e, Representative leaf phenotype of $\mathrm{C} 2$ and its mutant $\mathrm{C} 2$-Idf after rhizosphere transplantation. $\mathbf{f}$, Distinct bacterial diversity associated with $\mathrm{C} 2$ and its mutant rhizosphere microbiomes. $\mathbf{g}$. Differences in the relative abundance of bacterial families associated with $\mathrm{C} 2$ and mutant $C 2$-Idf rhizosphere microbiomes. $n=3$ biologically independent samples. Significances are calculated by multiple test correction (FDR adjusted ${ }^{*} p<0.05$, Welch's t-test). $\mathbf{h}$, Effects of exogenously applied flavonoid types on the variations of bacterial diversity of the C2-Idf mutant rhizosphere. i, Effects of exogenously applied flavonoid types 
on the family-level distribution (\%) of the bacterial community of the C2-Idf mutant rhizosphere. $n=5$ biologically independent samples. Different letters denote significance at $p<0.05$ (ANOVA, Tukey HSD).

Figure 4. Growth performance of mutants with lateral root defects is associated with flavoneconditioned rhizosphere microbiota. a, Shoot growth performance of root mutants in nitrogen-poor soil. $n=25$ biologically independent samples over three independent experiments. $\mathbf{b}$, Shoot nitrogen concentration and accumulation of root mutants in nitrogen-poor soil. $n=6$ biologically independent samples. c, Performance of lateral root defective mutants grown under in nitrogen-poor soil with and without subsequent sterilization. $n=10$ biologically independent samples. For each genotype, $p$ values were calculated using two-tailed unpaired $t$-tests according to the $F$-test results. $\mathbf{d}$, Targeted metabolite profiling indicates that Irt1 mutant produces and releases more flavone to the rhizosphere than the reference inbred line B73 and the mutant rum1. For root extracts and exudates, $n=4$ and $n=5$ biologically independent samples, respectively. Data are the mean \pm s.e.m. Soil in which flavonoid enriched plants were grown is able to promote Irt1 growth (e) and nitrogen acquisition (f) as demonstrated by rhizosphere transplantation. $n=5$ biologically independent samples. Experimental schemes of the rhizosphere transplantation of Irt 1 and rum 1 with different genotypes containing different concentrations of flavones are highlighted. For $\mathbf{a}, \mathbf{b}, \mathbf{d}, \mathbf{e}$ and $\mathbf{f}$, significances were indicated among different groups by exact $p$ values (ANOVA, Tukey HSD). For $\mathbf{a}, \mathbf{b}, \mathbf{c}, \mathbf{e}$ and $\mathbf{f}$, the boxes span from the first to the third quartiles, the centre lines represent the median values and the whiskers show the data that lie within the 1.5 interquartile range of the lower and upper quartiles. The data points at the ends of the whiskers represent the outliers.

Figure 5. Flavonoid-dependent Oxalobacteraceae promote plant growth and nitrogen acquisition by triggering developmental feedback on lateral root formation in nitrogen-poor soil. a, Shift of the rhizosphere bacterial $\alpha$-diversity (Shannon's diversity index) along the developmental zones for each mutant and B73. $n=3$ biologically independent samples. b. LEfSe cladogram indicating the phylogenetic distribution of bacterial lineages in the rhizosphere among different root mutants. Coloured circles show differentially abundant taxa and the colour is corresponding to the different root mutants in which the taxon displays the highest abundance. The six rings of the cladogram represent from inside to outside: domain, phylum, class, order, family, genus, and lineages with linear discriminant analysis (LDA) values $>4$ are displayed. Different letters of bar plot correspond to the bacterial taxa for class, order and family of the pie chart. c, Flavonoid-enriched plants grown soil is able to promote lateral root formation of Irt 1 by rhizosphere transplantation. A picture indicates more emerged lateral roots by rhizosphere transplantation of genotype 787 . $\mathbf{d}$, Correlation between lateral root density and shoot performance after inoculation with independent soil-derived Oxalobacteraceae isolates for Irt1 mutant grown in nitrogen-poor soil. Scatter plots show combined data from two independent inoculation experiments with best fit (solid line) and 95\% confidence interval (grey shade) for linear regression ( $p$ $<2.2 \times 10^{-16} ; n=10$; Supplementary Figure 17). For $\mathbf{c}$ and $\mathbf{d}, n=10$ biologically independent samples over two independent experiments. For $\mathbf{a}$ and $\mathbf{c}$, significances were indicated among different groups by exact $p$ values (ANOVA, Tukey HSD). The boxes span from the first to the third quartiles, the centre 
1 lines represent the median values and the whiskers show the data that lie within the 1.5 interquartile

2 range of the lower and upper quartiles. The data points at the ends of the whiskers represent the outliers.

3 Figure 6. Proposed model for flavone-dependent, microbiota-mediated lateral root formation

4 and plant performance. In nitrogen-poor soil, high content of flavone in root extracts and its exudates

5 could cause a compositional shift of the rhizosphere microbiota by enrichment of Oxalobacteraceae,

6 which indirectly boosts plant growth and nitrogen uptake by inducing lateral root formation in maize Irt1

7 mutant. 
2 We would like to thank P. Schulze-Lefert (Max Planck Institute for Plant Breeding Research, Cologne,

3 Germany) for the generous donation of the bacterial strains and natural soil for pot experiments. We

4 thank J. Birchler (University of Missouri, Columbia, USA), C. Gardener (United States Department of

5 Agriculture, Ames, US), P. S. Schnable (lowa State University, Ames, US), and T. Wang (Chinese

6 Academy of Agricultural Sciences, Beijing, China) for germplasm contribution. We thank Seon-Woo Lee

7 (Dong-A University, Busan, Republic of Korea) and Jihyun F Kim (Yonsei University, Seoul, Republic

8 of Korea) for sharing methods for rhizosphere transplantation. We thank C. Gutjahr (Technical

9 University of Munich, Munich, Germany), C. Knief (University of Bonn, Bonn, Germany) and B. Niu

10 (Northeast Forest University of China, Harbin, China) for valuable suggestions on the experiments. We

11 thank the student helpers from C. Zou's group at China Agricultural University (Beijing, China) for the

12 field sample harvest. We thank the technician Mrs. A. Glogau (University of Bonn, Bonn, Germany) for

13 nitrogen determination. This work is supported by DFG grants HO2249/9-3, HO2249/12-1 to F.H. and

14 YU272/1-1, Emmy Noether Programme (444755415) to P.Y., Germany's Excellence Strategy - EXC 152070 - PhenoRob grant 390732324 to G.S., Bundesministerium für Bildung und Forschung (BMBF) 16 grant 031B195C to F.H. and DFG Priority Program (SPP2089) "Rhizosphere Spatiotemporal 17 Organisation - a Key to Rhizosphere Functions" grant 403671039 to F.H. and P.Y. and 403670038 to 18 B.S.R. S.G.'s research is supported by Research Foundation - Flanders - Strategic Basic Research (grant no. 151553). K.T.'s research is supported by the Huazhong Agricultural University Scientific \& 20 Technological Self-innovation Foundation, the Max Planck Society and DFG grant SPP2125. X.C.'s research is supported by The Changjiang Scholarship, Ministry of Education, China, State Cultivation Base of Eco-agriculture for Southwest Mountainous Land (Southwest University, Chongqing, China), and the National Maize Production System in China (grant no. CARS-02-15). 

1. Brundrett, M. C. Coevolution of roots and mycorrhizas of land plants. New Phytol. 154, 275304 (2002).

2. Kenrick, P. \& Strullu-Derrien, C. The origin and early evolution of roots. Plant Physiol. 166, 570580 (2014).

3. Marschner P. 2012. Rhizosphere biology. In: Marschner P., ed. Marschner's mineral nutrition of higher plants, 3rd edn. San Diego: Academic Press, 369-388.

4. Berendsen, R. L., Pieterse, C. M. \& Bakker, P. A. The rhizosphere microbiome and plant health. Trends Plant Sci. 17, 478-486 (2012).

5. Mendes, R., Garbeva, P. \& Raaijmakers, J. M. The rhizosphere microbiome: significance of plant beneficial, plant pathogenic, and human pathogenic microorganisms. FEMS Microbiol. Rev. 37, 634-663 (2013).

6. Haney, C. H., Samuel, B. S., Bush, J. \& Ausubel, F. M. Associations with rhizosphere bacteria can confer an adaptive advantage to plants. Nat. Plants. 1, 15051 (2015).

7. Kwak, M. J. et al. Rhizosphere microbiome structure alters to enable wilt resistance in tomato. Nat. Biotechnol. 36, 1100 (2018).

8. Lu, T. et al. Rhizosphere microorganisms can influence the timing of plant flowering. Microbiome 6, 231 (2018).

9. Bulgarelli, D. et al. Revealing structure and assembly cues for Arabidopsis root-inhabiting bacterial microbiota. Nature 488, 91 (2012).

10. Lundberg, D. S. et al. Defining the core Arabidopsis thaliana root microbiome. Nature 488, 86 (2012).

11. Schreiter, S. et al. Effect of the soil type on the microbiome in the rhizosphere of field-grown lettuce. Front. Microbiol. 5, 144 (2014).

12. Veach, A. M. et al. Rhizosphere microbiomes diverge among Populus trichocarpa plant-host genotypes and chemotypes, but it depends on soil origin. Microbiome 7, 76 (2019).

13. Turner, T. R., James, E. K. \& Poole, P. S. The plant microbiome. Genome Biol. 14, 209 (2013).

14. Ofek-Lalzar, M. et al. Niche and host-associated functional signatures of the root surface microbiome. Nat. Commun. 5, 4950 (2014).

15. Fitzpatrick, C. R. et al. Assembly and ecological function of the root microbiome across angiosperm plant species. Proc. Natl Acad. Sci. USA 115, E1157-E1165 (2018).

16. Gruber, B., Giehl, R., Friedel, S. \& von Wirén N. Plasticity of the Arabidopsis root system under nutrient deficiencies. Plant Physiol. 163, 161-179 (2013).

17. Garrido-Oter, R. et al. Modular traits of the rhizobiales root microbiota and their evolutionary relationship with symbiotic rhizobia. Cell Host Microbe 24, 155-167 (2018).

18. Verbon, E. H. \& Liberman, L. M. Beneficial microbes affect endogenous mechanisms controlling root development. Trends Plant Sci. 21, 218-229 (2016).

19. Hochholdinger, F., Yu, P. \& Marcon, C. Genetic control of root system development in maize. Trends Plant Sci. 23, 79-88 (2018).

20. Hake, S. \& Ross-Ibarra, J. The natural history of model organisms: genetic, evolutionary and plant breeding insights from the domestication of maize. Elife 4, e05861 (2015).

21. Yu, P., Gutjahr, C., Li, C. \& Hochholdinger, F. Genetic control of lateral root formation in cereals. Trends Plant Sci. 21, 951-961 (2016a).

22. Tai, H. et al. Transcriptomic and anatomical complexity of primary, seminal, and crown roots highlight root type-specific functional diversity in maize (Zea mays L.). J. Exp. Bot. 67, 11231135 (2015).

23. Yu, P., Eggert, K., von Wirén, N., Li, C. \& Hochholdinger, F. Cell type-specific gene expression analyses by RNA sequencing reveal local high nitrate-triggered lateral root initiation in shootborne roots of maize by modulating auxin-related cell cycle regulation. Plant Physiol. 169, 690704 (2015).

24. $\mathrm{Yu}$, P. et al. Root type-specific reprogramming of maize pericycle transcriptomes by local high nitrate results in disparate lateral root branching patterns. Plant Physiol. 170, 1783-1798 (2016b). 
25. Szoboszlay, M. et al. Comparison of root system architecture and rhizosphere microbial communities of Balsas teosinte and domesticated corn cultivars. Soil Biol. Biochem. 80, 34-44 (2015).

26. Gutjahr, C. et al. Transcriptome diversity among rice root types during asymbiosis and interaction with arbuscular mycorrhizal fungi. Proc. Natl Acad. Sci. USA 112, 6754-6759 (2015).

27. Yu, P. et al. Root type and soil phosphate determine the taxonomic landscape of colonizing fungi and the transcriptome of field-grown maize roots. New Phytol. 217, 1240-1253 (2018).

28. Cotton, T. A. et al. Metabolic regulation of the maize rhizobiome by benzoxazinoids. ISME J. 13, 1647-1658 (2019).

29. Zhang, J. et al. NRT1. $1 B$ is associated with root microbiota composition and nitrogen use in field-grown rice. Nat. Biotechnol. 37, 676-684 (2019).

30. Peiffer, J. A. et al. Diversity and heritability of the maize rhizosphere microbiome under field conditions. Proc. Natl Acad. Sci. USA 110, 6548-6553 (2013).

31. Walters, W. A. et al. Large-scale replicated field study of maize rhizosphere identifies heritable microbes. Proc. Natl Acad. Sci. USA 115, $7368-7373$ (2018).

32. Cesco, S., Neumann, G., Tomasi, N., Pinton, R. \& Weisskopf, L. Release of plant-borne flavonoids into the rhizosphere and their role in plant nutrition. Plant Soil 329, 1-25 (2010).

33. $\mathrm{Hu}$, L. et al. Root exudate metabolites drive plant-soil feedbacks on growth and defense by shaping the rhizosphere microbiota. Nat. Commun. 9, 2738 (2018).

34. Kudjordjie, E. N., Sapkota, R., Steffensen, S. K., Fomsgaard, I. S. \& Nicolaisen, M. Maize synthesized benzoxazinoids affect the host associated microbiome. Microbiome 7, 59 (2019).

35. Hassan, S. \& Mathesius, U. The role of flavonoids in root-rhizosphere signalling: opportunities and challenges for improving plant-microbe interactions. J. Exp. Bot. 63, 3429-3444 (2012).

36. Mierziak, J., Kostyn, K. \& Kulma, A. Flavonoids as important molecules of plant interactions with the environment. Molecules 19, 16240-16265 (2014).

37. Ferreyra, M. L. F. et al. The identification of maize and Arabidopsis type I flavone synthases links flavones with hormones and biotic interactions. Plant Physiol. 169, 1090-1107 (2015).

38. Eloy, N. B. et al. Silencing CHALCONE SYNTHAS E in maize impedes the incorporation of tricin into lignin and increases lignin content. Plant Physiol. 173, 998-1016 (2017).

39. Righini, S., Rodriguez, E.J., Berosich, C., Grotewold, E., Casati, P. \& Falcone Ferreyra, M.L. Apigenin produced by maize flavone synthase I and II protects plants against UV-B-induced damage. Plant Cell Environ. 42, 495-508 (2019).

40. Wasson, A. P., Pellerone, F. I. \& Mathesius, U. Silencing the flavonoid pathway in Medicago truncatula inhibits root nodule formation and prevents auxin transport regulation by rhizobia. Plant Cell 18, 1617-1629 (2006).

41. Subramanian, S., Stacey, G. \& Yu, O. Distinct, crucial roles of flavonoids during legume nodulation. Trends Plant Sci. 12, 282-285 (2007).

42. Oldroyd, G. E. \& Leyser, O. A plant's diet, surviving in a variable nutrient environment. Science 368, eaba0196 (2020).

43. Zhang, J., Subramanian, S., Stacey, G., \& Yu, O. Flavones and flavonols play distinct critical roles during nodulation of Medicago truncatula by Sinorhizobium meliloti. Plant J. 57, 171-183 (2009).

44. de Vries, F. T., Griffiths, R. I., Knight, C. G., Nicolitch, O. \& Williams, A. Harnessing rhizosphere microbiomes for drought-resilient crop production. Science 368, 270-274 (2020).

45. Barberon, M. The endodermis as a checkpoint for nutrients. New Phytol. 213, 1604-1610 (2017).

46. Duan, F., Giehl, R. F. H., Geldner, N., Salt, D. E. \& von Wirén, N. Root zone-specific localization of AMTs determines ammonium transport pathways and nitrogen allocation to shoots. PLoS Biol. 16, e2006024 (2018).

47. Giehl, R. F. \& von Wirén, N. Root nutrient foraging. Plant Physiol. 166, 509-517 (2014).

48. Jia, Z., Giehl, R. F. H., Meyer, R. C., Altmann, T. \& von Wirén, N. Natural variation of BSK3 tunes brassinosteroid signaling to regulate root foraging under low nitrogen. Nat. Commun. 10, 2378 (2019).

49. Postma, J. A., Dathe, A. \& Lynch, J. P. The optimal lateral root branching density for maize depends on nitrogen and phosphorus availability. Plant Physiol. 166, 590-602 (2014). 
50. Zhan, A. \& Lynch, J. P. Reduced frequency of lateral root branching improves $\mathrm{N}$ capture from low-N soils in maize. J. Exp. Bot. 66, 2055-2065 (2015).

51. Badri, D. V. \& Vivanco, J. M. Regulation and function of root exudates. Plant Cell Environ. 32 , 666-681 (2009).

52. Sasse, J., Martinoia, E. \& Northen, T. Feed your friends: do plant exudates shape the root microbiome? Trends Plant Sci. 23, 25-41 (2018).

53. Stringlis, I. A. et al. MYB72-dependent coumarin exudation shapes root microbiome assembly to promote plant health. Proc. Natl Acad. Sci. USA 115, E5213-E5222 (2018).

54. Voges, M. J., Bai, Y., Schulze-Lefert, P. \& Sattely, E. S. Plant-derived coumarins shape the composition of an Arabidopsis synthetic root microbiome. Proc. Natl Acad. Sci. USA 116, 12558-12565 (2019).

55. Lebeis, S. L. et al. Salicylic acid modulates colonization of the root microbiome by specific bacterial taxa. Science 349, 860-864 (2015).

56. Bulgarelli, D. et al. Structure and function of the bacterial root microbiota in wild and domesticated barley. Cell Host Microbe 17, 392-403 (2015).

57. Beirinckx, S. et al. Tapping into the maize root microbiome to identify bacteria that promote growth under chilling conditions. Microbiome 8, 54 (2020).

58. Stelpflug, S. C. et al. An expanded maize gene expression atlas based on RNA sequencing and its use to explore root development. Plant Genome 9, 1-15 (2016).

59. Ofek, M., Hadar, Y. \& Minz, D. Ecology of root colonizing Massilia (Oxalobacteraceae). PloS One 7, p.e40117 (2012).

60. Gutiérrez-Luna, F. M. et al. Plant growth-promoting rhizobacteria modulate root-system architecture in Arabidopsis thaliana through volatile organic compound emission. Symbiosis 51, 75-83 (2010).

61. Poitout, A. et al. Local signalling pathways regulate the Arabidopsis root developmental response to Mesorhizobium loti inoculation. J. Exp. Bot. 68, 1199-1211 (2017).

62. López-Bucio, J. et al. Bacillus megaterium rhizobacteria promote growth and alter root-system architecture through an auxin- and ethylene-independent signaling mechanism in Arabidopsis thaliana. Mol. Plant-Microbe Interact. 20, 207-217 (2007).

63. Finkel, O. M. et al. A single bacterial genus maintains root development in a complex microbiome. Nature 587, 103-108 (2020).

64. Schiessl, K. et al. NODULE INCEPTION recruits the lateral root developmental program for symbiotic nodule organogenesis in Medicago truncatula. Curr. Biol. 29, 3657-3668 (2019).

65. Soyano, T., Shimoda, Y., Kawaguchi, M. \& Hayashi, M. A shared gene drives lateral root development and root nodule symbiosis pathways in Lotus. Science 366, 1021-1023 (2019).

66. Zhu, F. et al. A CEP peptide receptor-like kinase regulates auxin biosynthesis and ethylene signaling to coordinate root growth and symbiotic nodulation in Medicago truncatula. Plant Cell doi:10.1105/tpc.19.00428 (2020).

67. Romay, M. C.et al. Comprehensive genotyping of the USA national maize inbred seed bank. Genome Biol. 14, R55 (2013).

68. Della Vedova, C. B. et al. The dominant inhibitory chalcone synthase allele C2-Idf (inhibitor diffuse) from Zea mays (L.) acts via an endogenous RNA silencing mechanism. Genetics 170, 1989-2002 (2005).

69. Bertin, P. \& Gallais, A. Genetic variation for nitrogen use efficiency in a set of recombinant maize inbred lines. I. Agrophysiological results. Maydica 45, 53-66 (2000).

70. Nelson, D. W. \& Sommers, L. E. Determination of total nitrogen in plant material. Agron. J. 65, 109-112 (1973).

71. Edwards, J. et al. Structure, variation, and assembly of the root-associated microbiomes of rice. Proc. Natl Acad. Sci. USA 112, E911-E920 (2015).

72. Smyth, G. K. Linear models and empirical Bayes methods for assessing differential expression in microarray experiments. Stat. Appl. Genet. Mol. Biol. 3, Article 3 (2004).

73. Benjamini, Y. \& Hochberg, Y. Controlling the false discovery rate: a practical and powerful approach to multiple testing. J. R. Stat. Soc. B. 57, 289-300 (1995).

74. Tian, T. et al. agriGO v2. 0: a GO analysis toolkit for the agricultural community, 2017 update. Nucleic Acids Res. 45, W122-W129 (2017). 
75. Caporaso, J. G. et al. Global patterns of $16 \mathrm{~S}$ rRNA diversity at a depth of millions of sequences per sample. Proc. Natl Acad. Sci. USA 108, 4516-4522 (2011).

76. Magoč, T. \& Salzberg, S. L. FLASH: fast length adjustment of short reads to improve genome assemblies. Bioinformatics 27, 2957-2963 (2011).

77. Caporaso, J. G. et al. QIIME allows analysis of high-throughput community sequencing data. Nat. Methods. 7, 335 (2010).

78. Bokulich, N. A. et al. Quality-filtering vastly improves diversity estimates from Illumina amplicon sequencing. Nat. Methods 10, 57 (2013).

79. Edgar, R. C., Haas, B. J., Clemente, J. C., Quince, C. \& Knight, R. UCHIME improves sensitivity and speed of chimera detection. Bioinformatics 27, 2194-2200 (2011).

80. Haas, B. J. et al. Chimeric 16S rRNA sequence formation and detection in Sanger and 454pyrosequenced PCR amplicons. Genome Res. 21, 494-504 (2011).

81. Edgar, R. C. UPARSE: highly accurate OTU sequences from microbial amplicon reads. Nat. Methods. 10, 996 (2013).

82. Wang, Q., Garrity, G. M., Tiedje, J. M. \& Cole, J. R. Naive Bayesian classifier for rapid assignment of rRNA sequences into the new bacterial taxonomy. Appl. Environ. Microb. 73, 5261-5267 (2007).

83. Quast, C. et al. The SILVA ribosomal RNA gene database project: improved data processing and web-based tools. Nucleic Acids Res. 41, D590-D596 (2013).

84. Edgar, R. C. MUSCLE: multiple sequence alignment with high accuracy and high throughput. Nucleic Acids Res. 32, 1792-1797 (2004).

85. Kõljalg, U. et al. Towards a unified paradigm for sequence-based identification of fungi. Mol. Ecol. 22, 5271-5277 (2013).

86. Lê, S., Josse, J. \& Husson, F. FactoMineR: an R package for multivariate analysis. J. Stat. Softw. 25, 1-18 (2008).

87. Segata, N. \& Huttenhower, C. Toward an efficient method of identifying core genes for evolutionary and functional microbial phylogenies. PLoS One 6, e24704 (2011).

88. Durán, P. et al. Microbial interkingdom interactions in roots promote Arabidopsis survival. Cell 175, 973-983 (2018).

89. Faust, K. \& Raes, J. CoNet app: inference of biological association networks using Cytoscape. F1000Res 5, 1519 (2016).

90. Shannon, P. et al. Cytoscape: a software environment for integrated models of biomolecular interaction networks. Genome Res.13, 2498-2504 (2003).

91. Friedman, J. \& Alm, E. J. Inferring correlation networks from genomic survey data. PLoS. Comput. Biol. 8, e1002687 (2012).

92. Wang, Q., Wang, K., Wu, W., Giannoulatou, E., Ho, J. W. \& Li, L. Host and microbiome multiomics integration: applications and methodologies. Biophys. Rev. 11, 55-65 (2019).

93. Langfelder, P. \& Horvath, S. WGCNA: an R package for weighted correlation network analysis. BMC Bioinformatics 9, 559 (2008).

94. Zhang, B. \& Horvath, S. A general framework for weighted gene co-expression network analysis. Stat. Appl. Genet. Mol. 4 (2005).

95. Supek, F., Bošnjak, M., Škunca, N. \& Šmuc, T. REVIGO summarizes and visualizes long lists of gene ontology terms. PLoS One 6, e21800 (2011).

96. Tatusov, R. L. et al. The COG database: an updated version includes eukaryotes. BMC Bioinformatics 4, 1-14 (2003).

97. Stiehl-Braun, P. A., Hartmann, A. A., Kandeler, E., Buchmann, N. I. N. A. \& Niklaus, P. A. Interactive effects of drought and $\mathrm{N}$ fertilization on the spatial distribution of methane assimilation in grassland soils. Global Change Biol. 17, 2629-2639 (2011).

98. Glickmann, E. \& Dessaux, Y. A critical examination of the specificity of the salkowski reagent for indolic compounds produced by phytopathogenic bacteria. Appl. Environ. Microbiol. 61, 793796 (1995). 
Emerged

Rhizosphere Lateral root Root hairs lateral roots
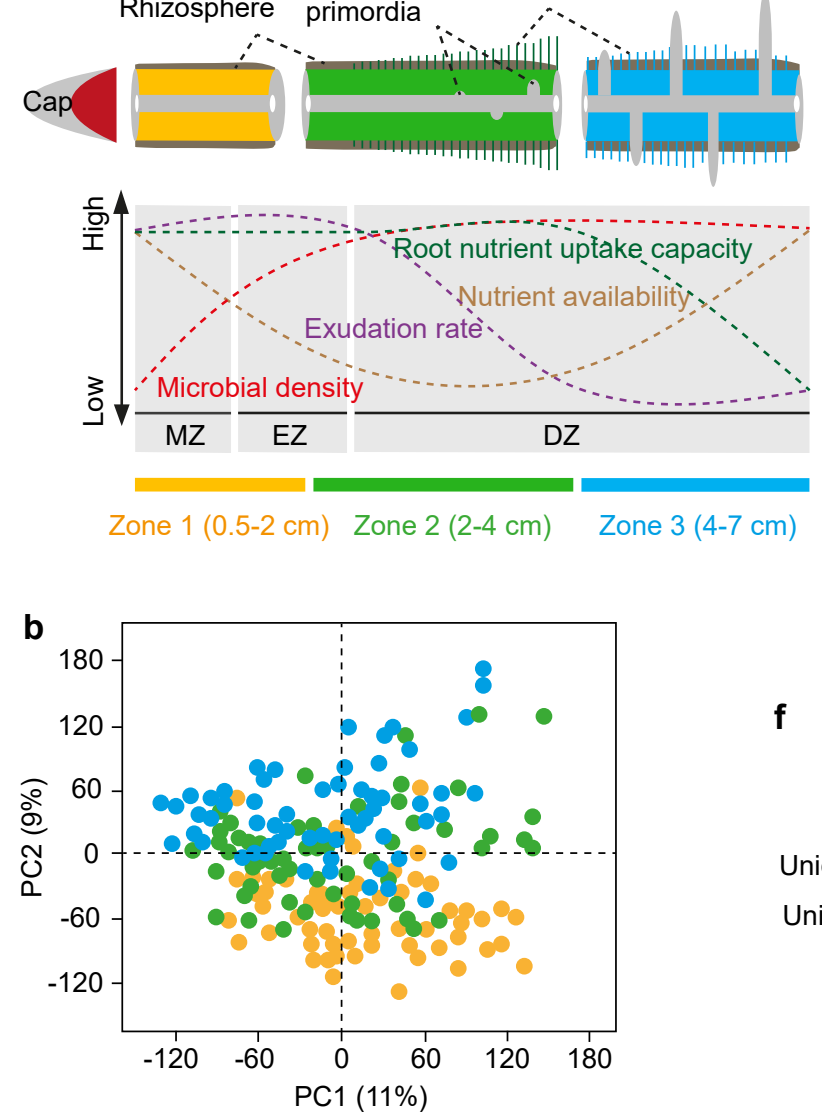

d
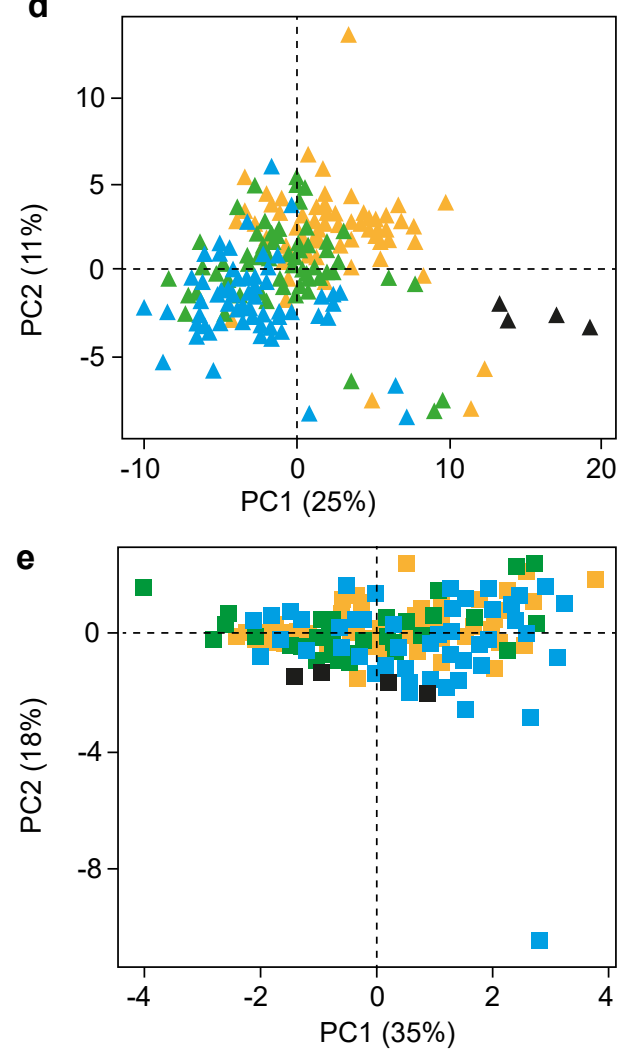

\begin{tabular}{|lccc|}
\hline \multicolumn{2}{|c|}{ Root } & \multicolumn{2}{c|}{ Rhizosphere } \\
Transcriptome & Bacteria & Fungi \\
Zone 1 & 0 & $\Delta$ & \\
Zone 2 & $\bullet$ & $\Delta$ & $\square$ \\
Zone 3 & $\bullet$ & $\Delta$ & $\square$ \\
Bulk soil & & $\Delta$ & $\square$ \\
\hline
\end{tabular}

C Root system development
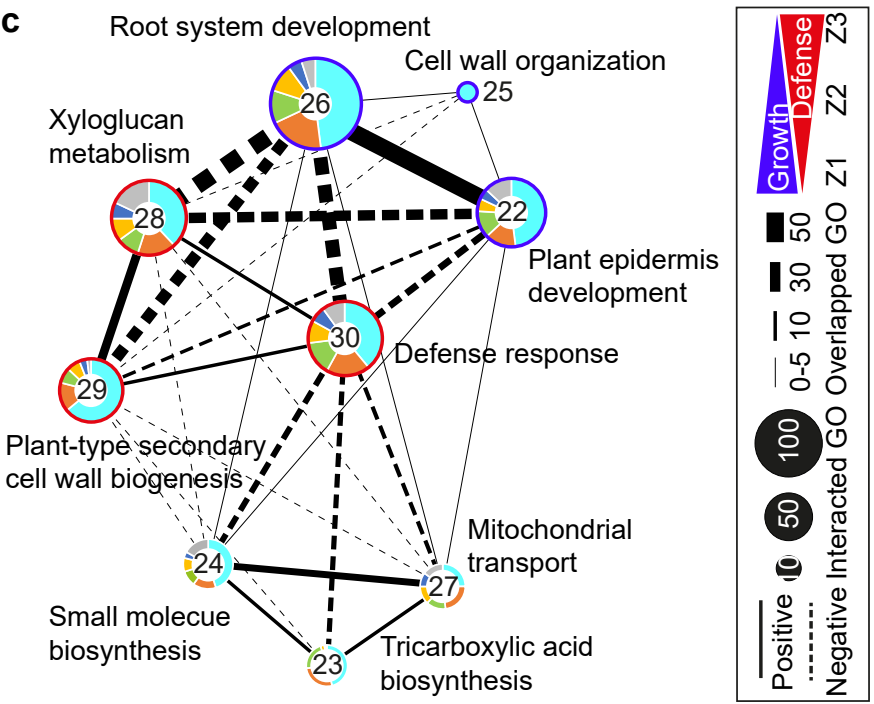

Total host expressed genes interacting with bacteria and fungi: 7,533
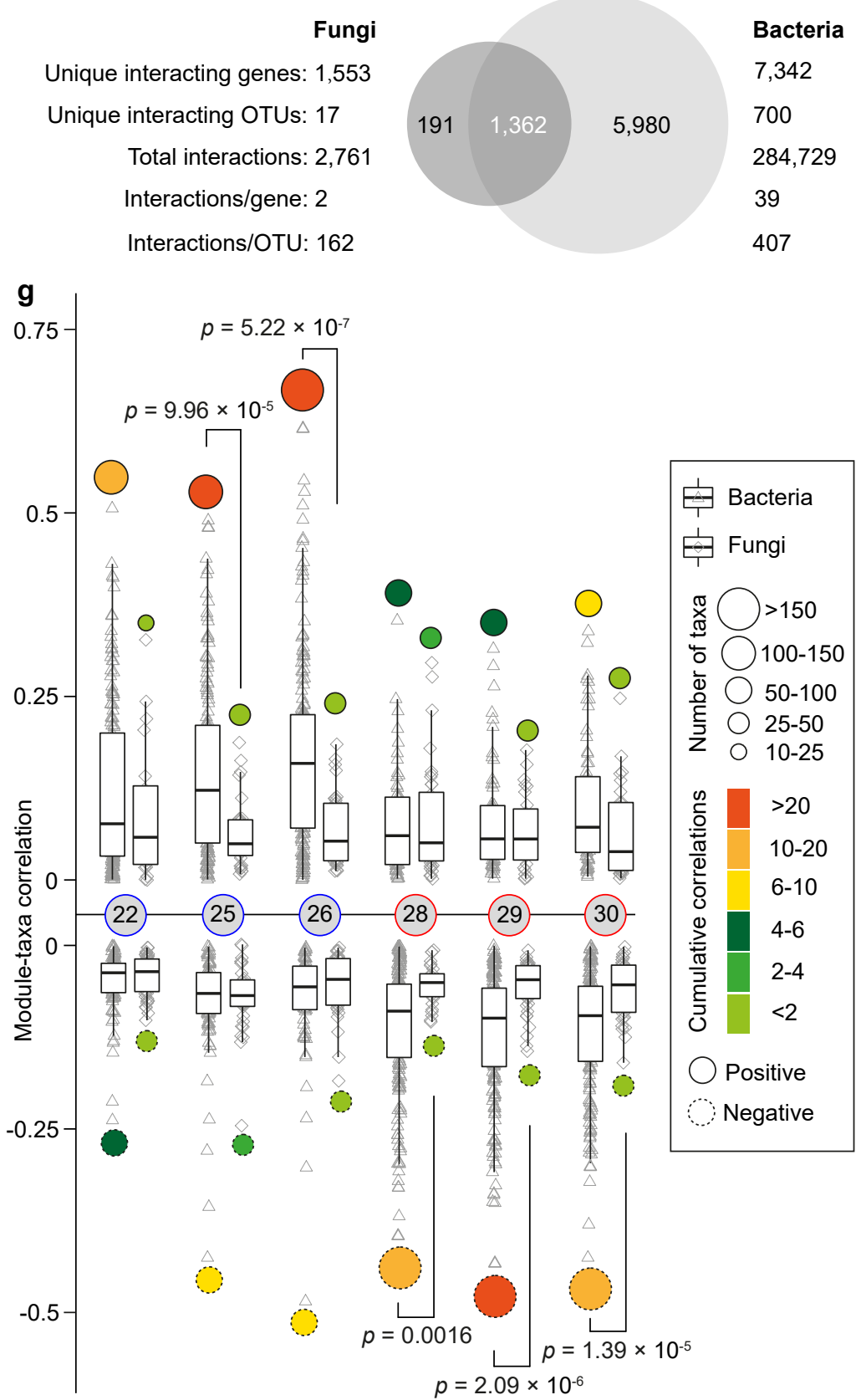

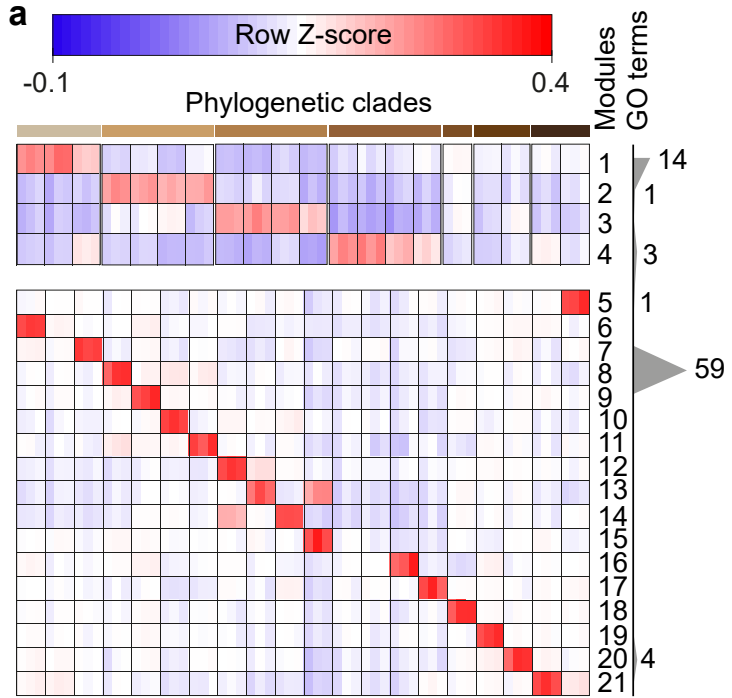

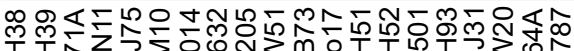

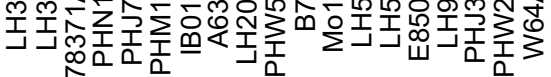

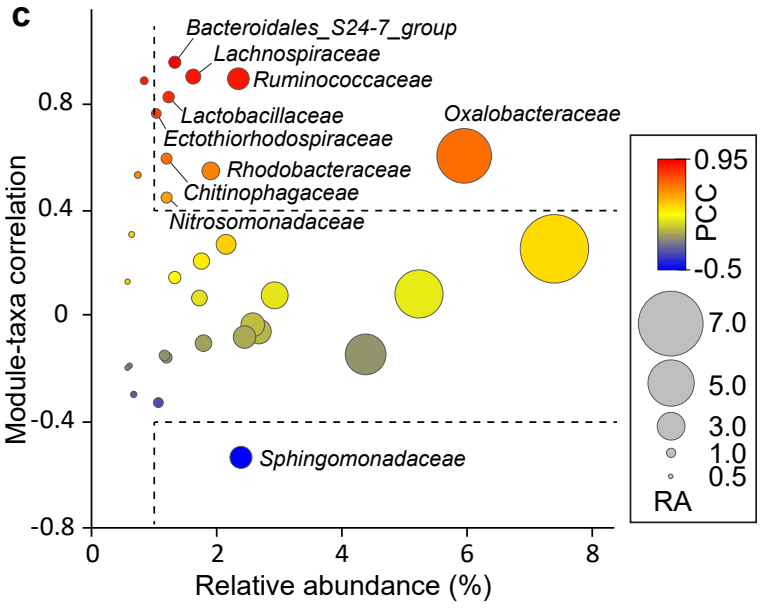

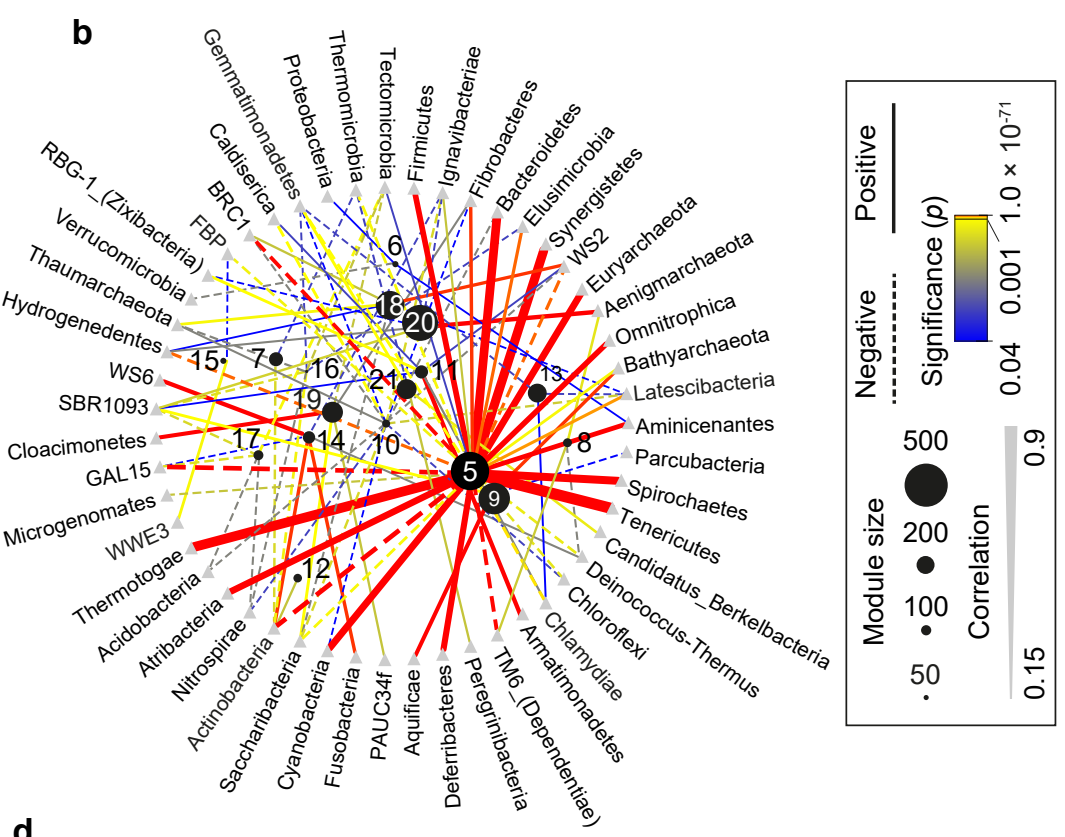

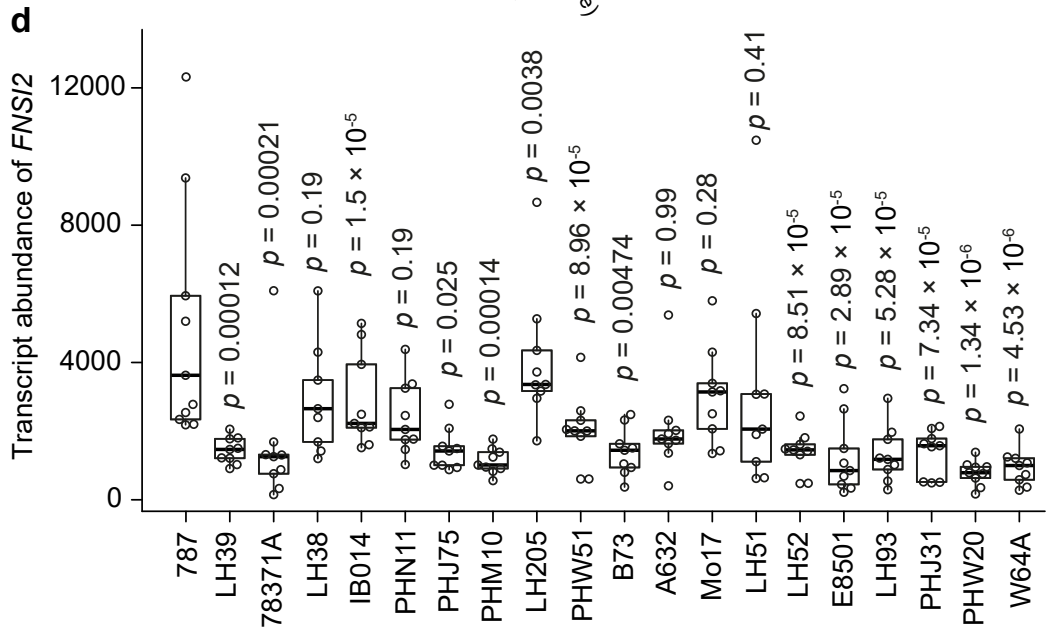

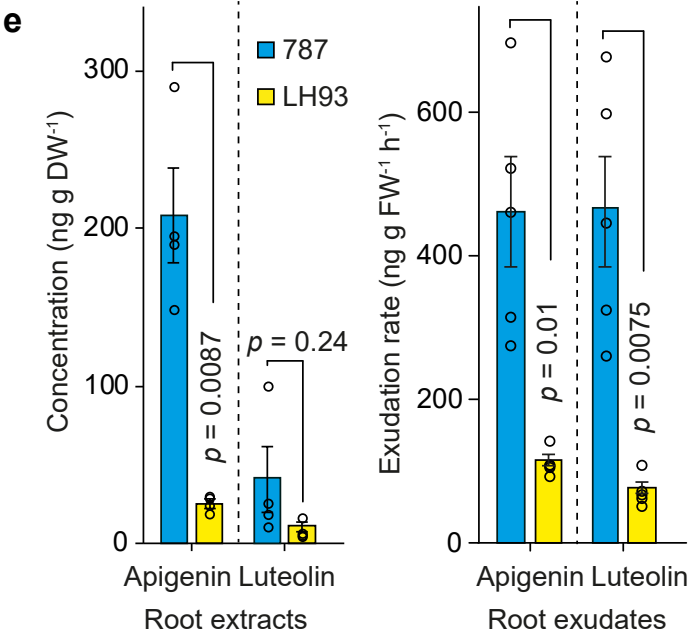
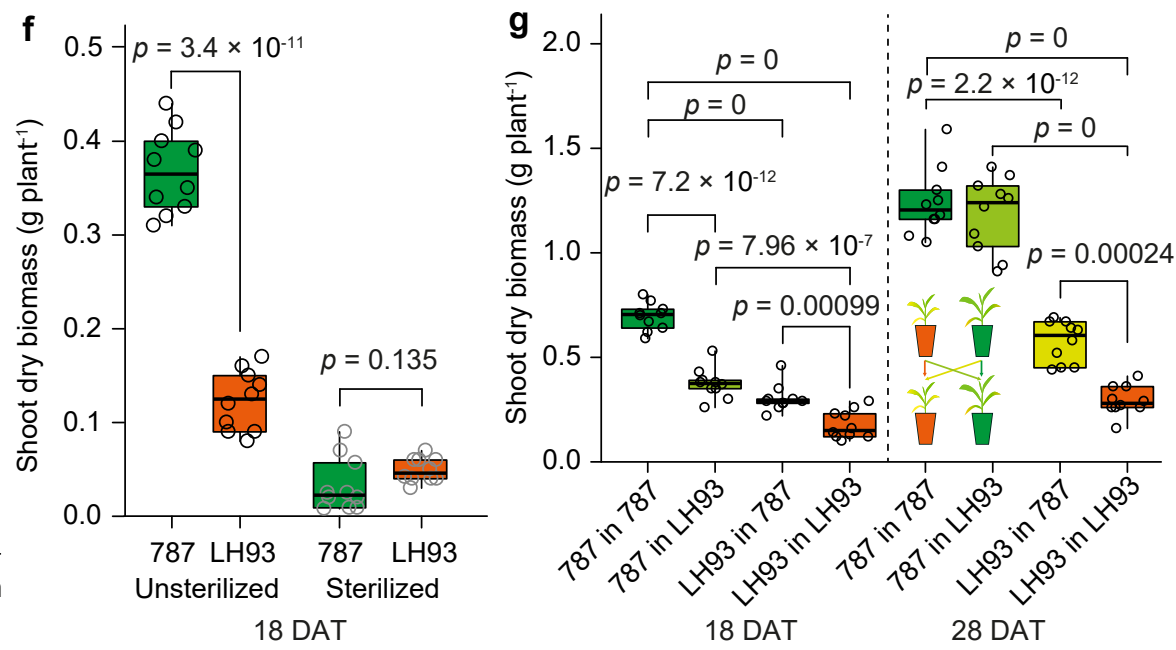

Figure 2 
a Acetyl-CoA

b

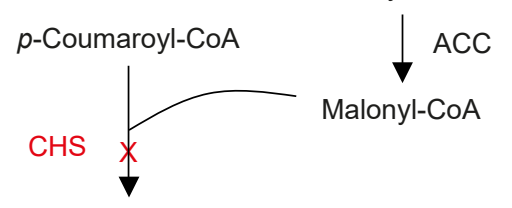

Chalcone

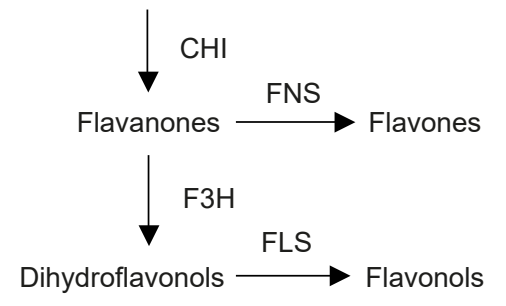

$\frac{\stackrel{4}{\mathbb{N}}}{\stackrel{4}{9}}$

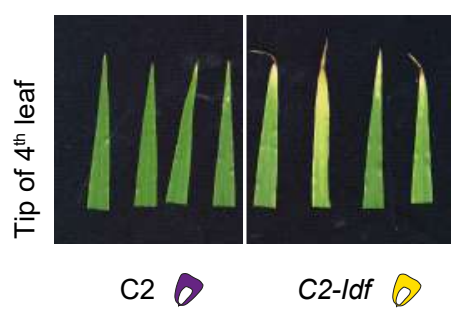

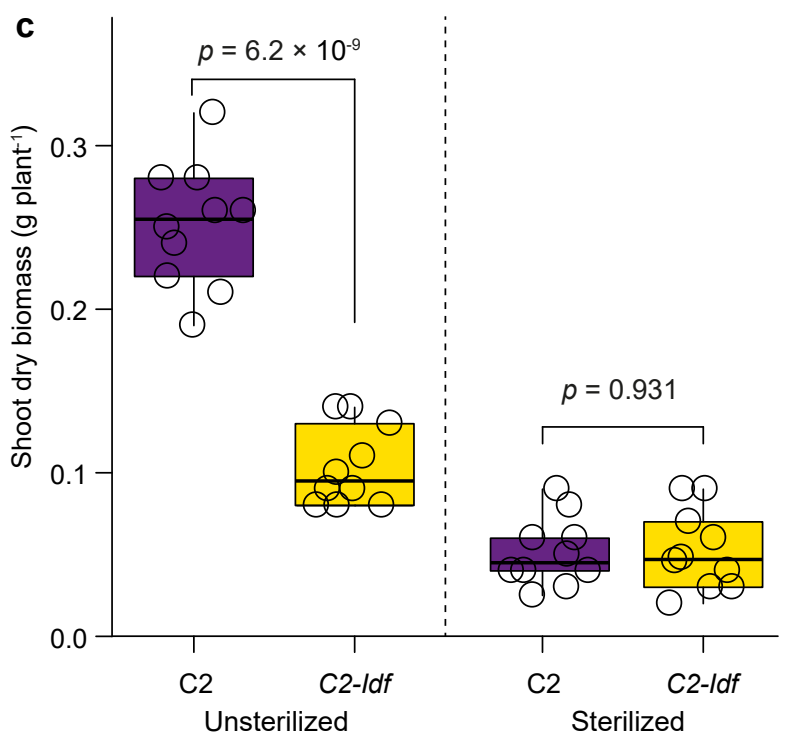
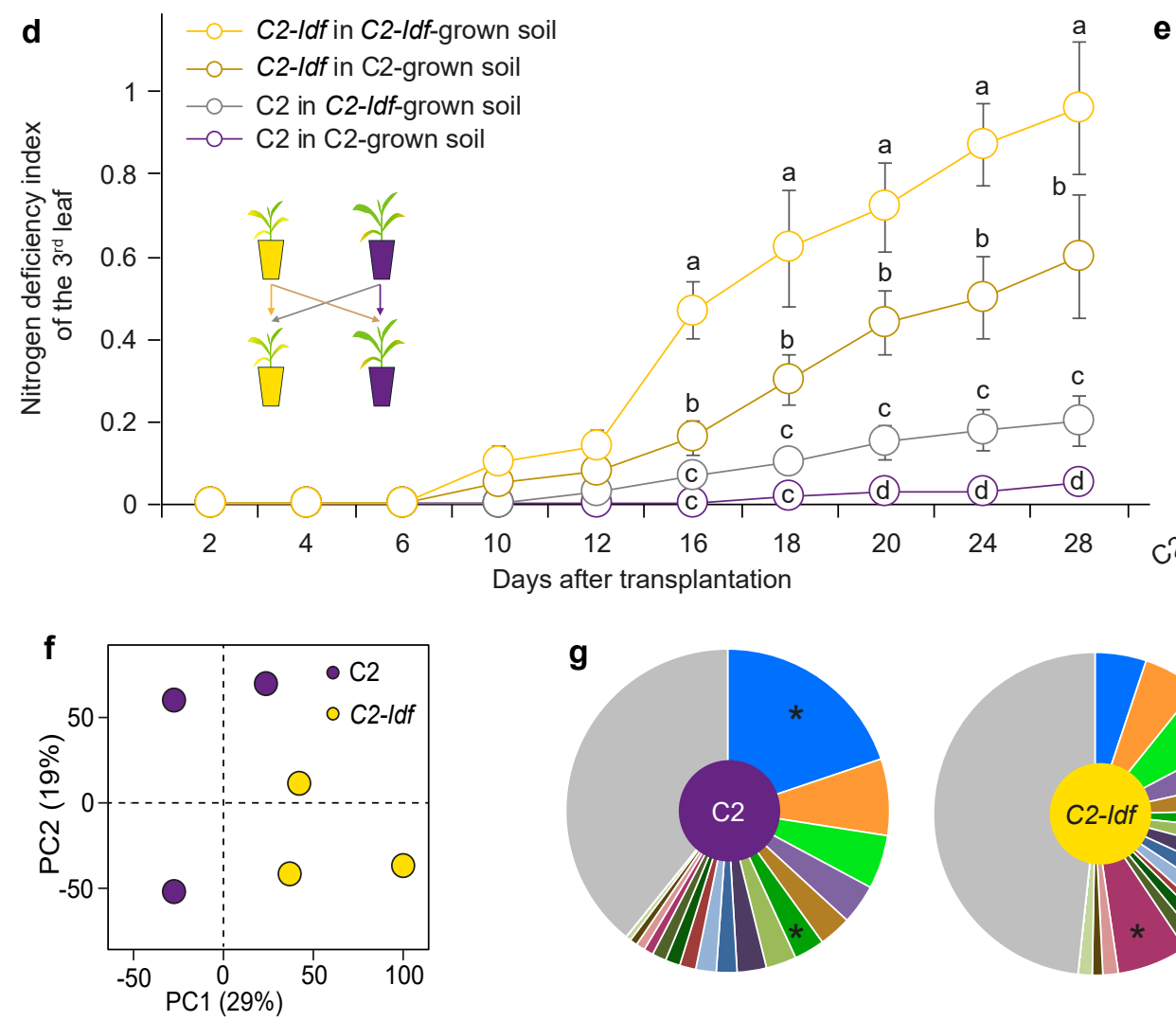

h

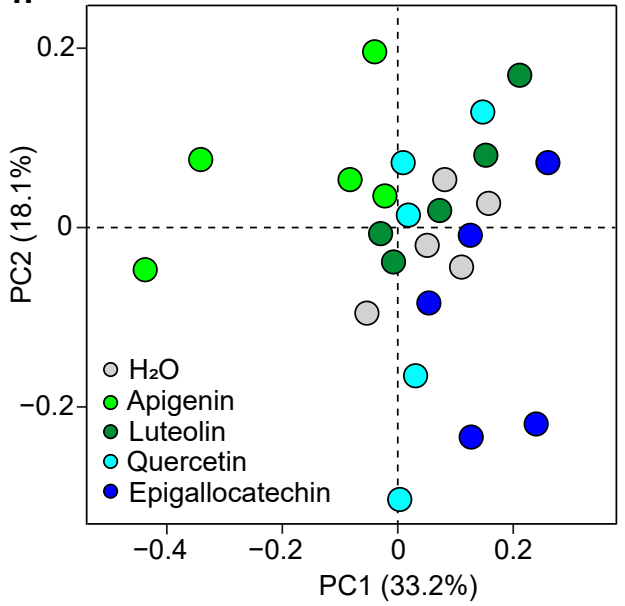

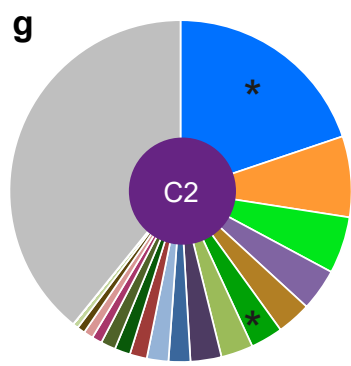
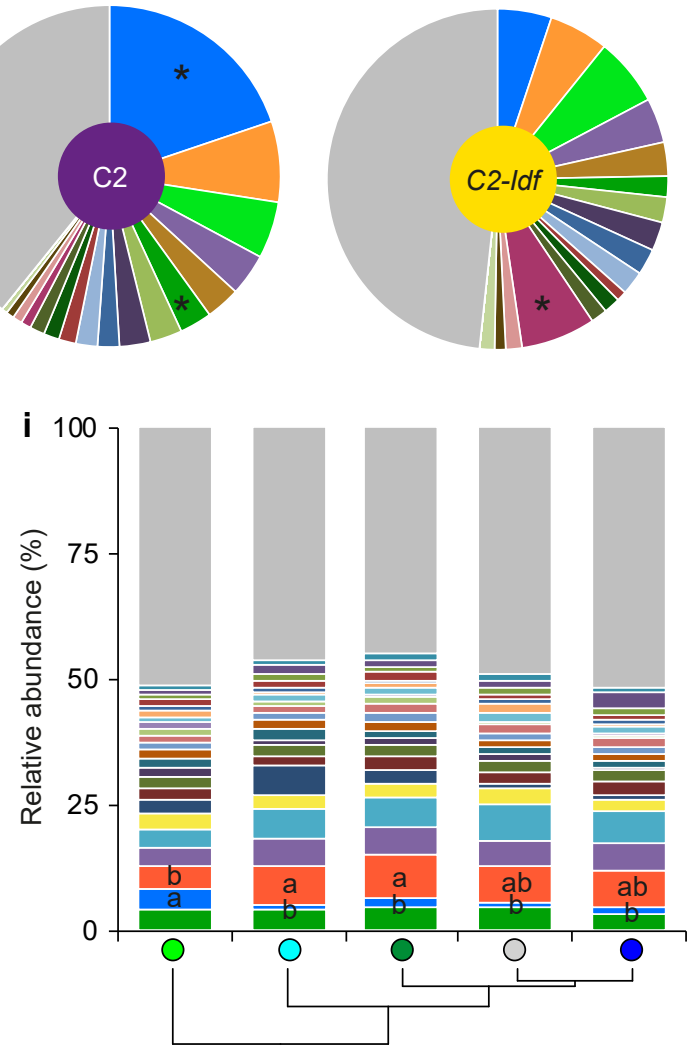

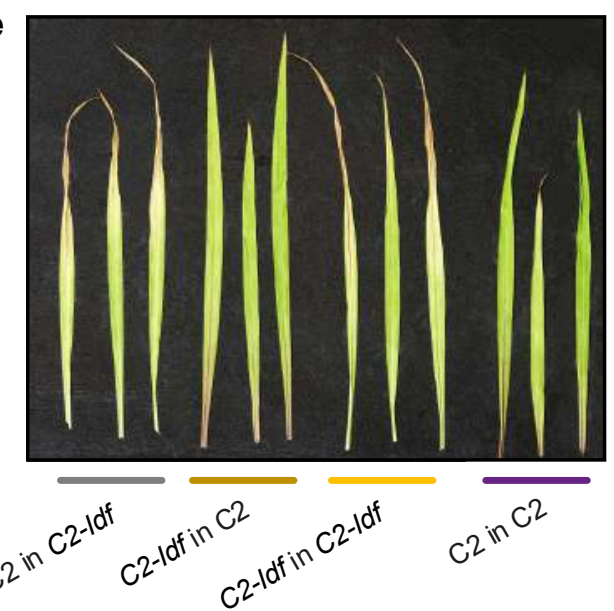

Others

- unidentified_Rhizobiales

unidentified_Xanthomonadales

DA111

Ellin6075

$m b 2424$

Bacillaceae

Nocardioidaceae

- Solibacteraceae

- Phyllobacteriaceae

- Chitinophagaceae

Moraxellaceae

- Syntrophobacteraceae

Gemmataceae

Enterobacteriaceae

- Gaiellaceae

Rhizobiaceae

- Micrococcaceae

- Cytophagaceae

- Paenibacillaceae

- Rhodospirillaceae

Hyphomicrobiaceae

- Pseudomonadaceae

Sinobacteraceae

- Nitrosomonadaceae

- Streptomycetaceae

- Sphingobacteriaceae

Gemmatimonadaceae

- Acidobacteriaceae

- Verrucomicrobiaceae

- Xanthomonadaceae

- Sphingomonadaceae

Oxalobacteraceae

- Comamonadaceae 

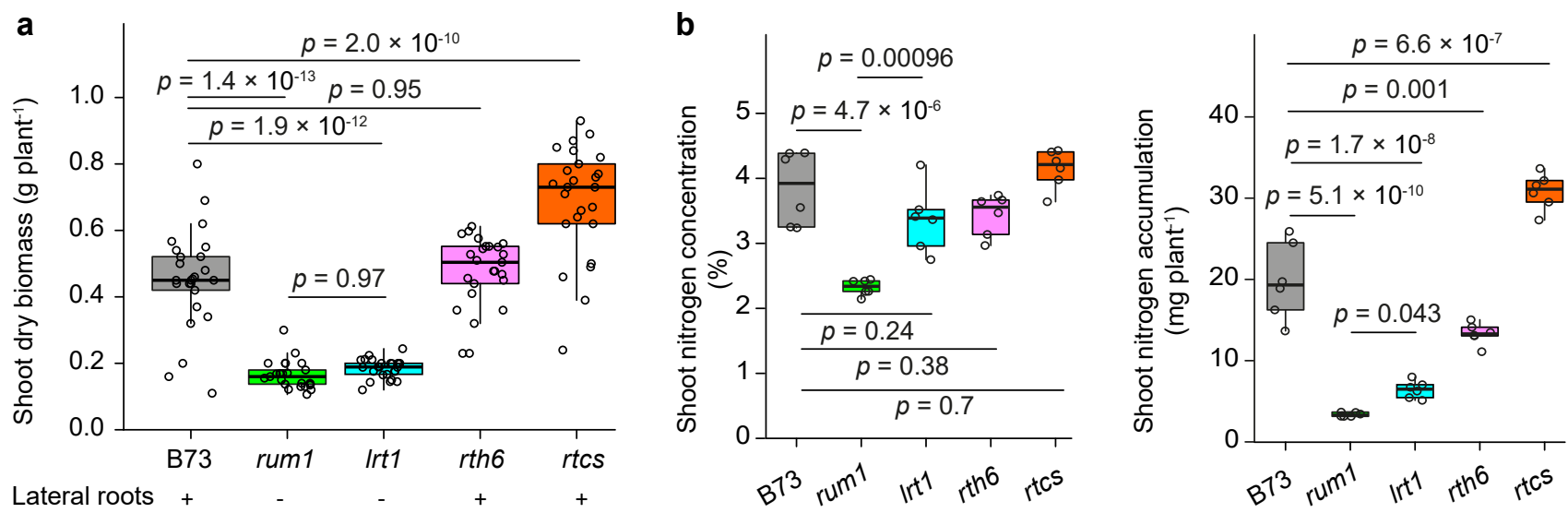
$\begin{array}{llllll}\text { Lateral roots }+ & - & - & + & + \\ \text { Root hairs } & + & + & + & - & + \\ \text { Seminal roots }+ & - & + & + & -\end{array}$
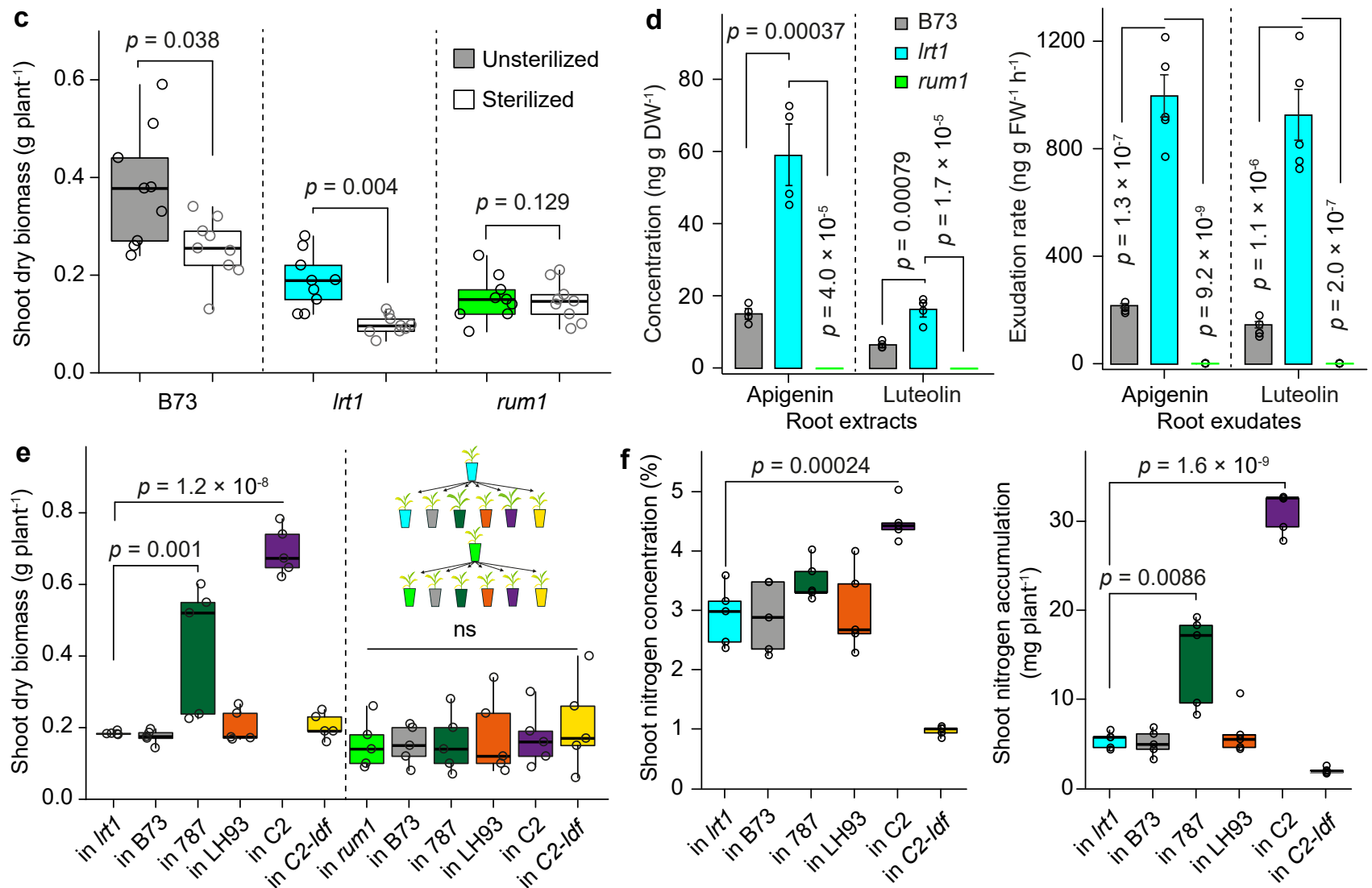

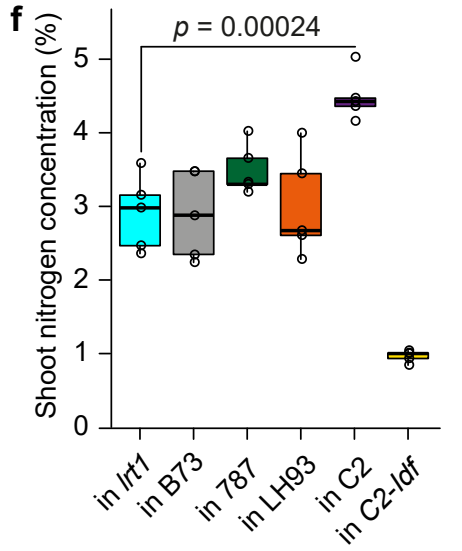

Irt1 transplantation

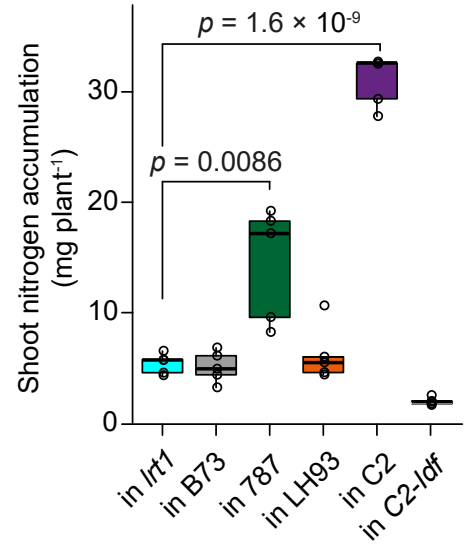

Irt1 transplantation 



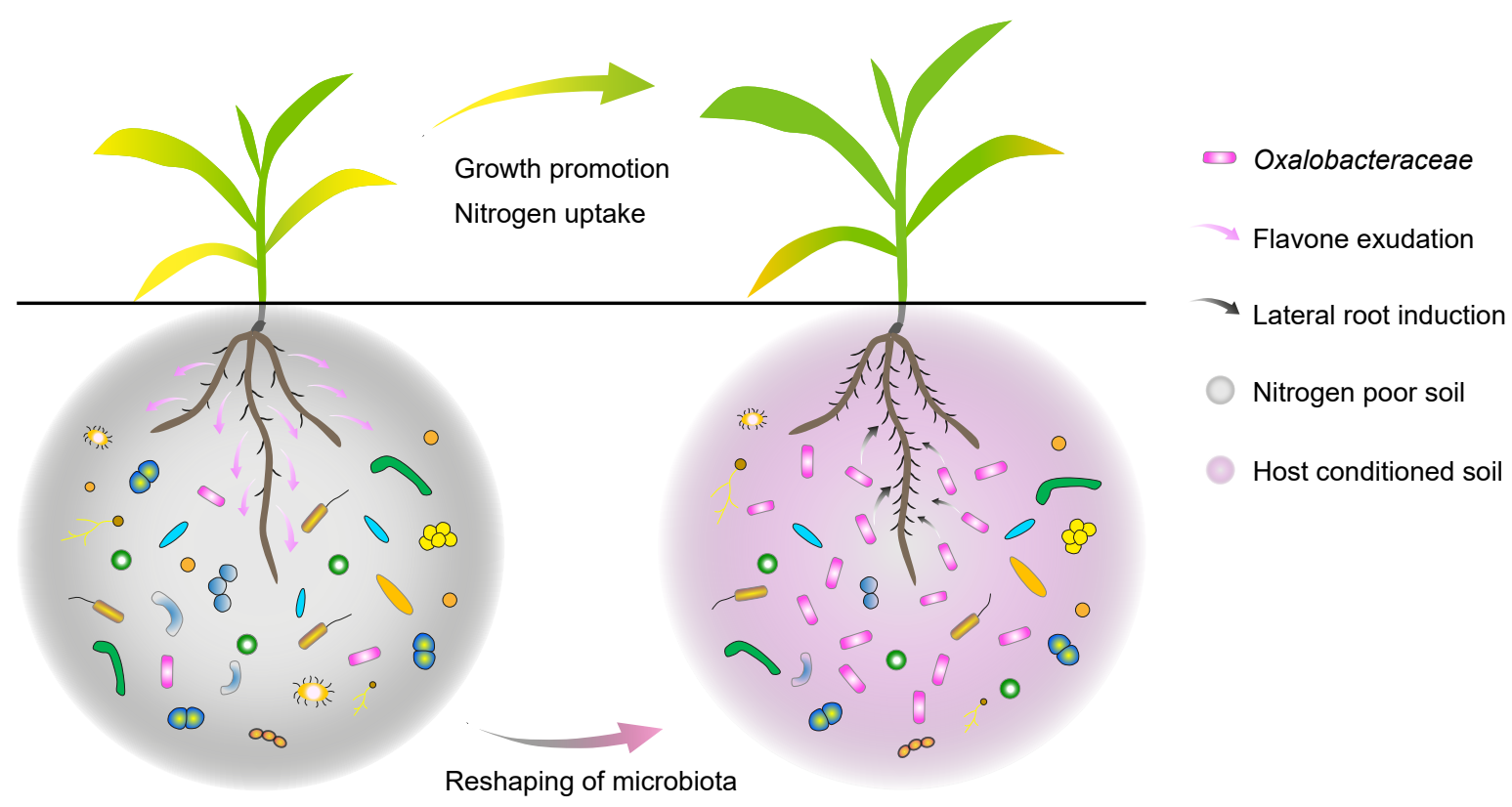

Figure 6 


\section{Supplementary Figures and Tables}

a

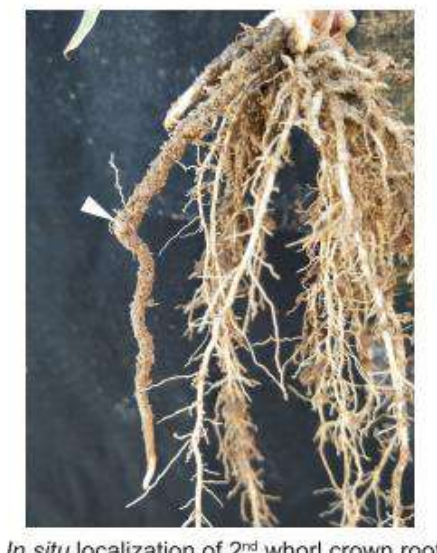

In situ localization of $2^{\text {nd }}$ whorl crown root

C $\quad 5,066$ differentially expressed genes b

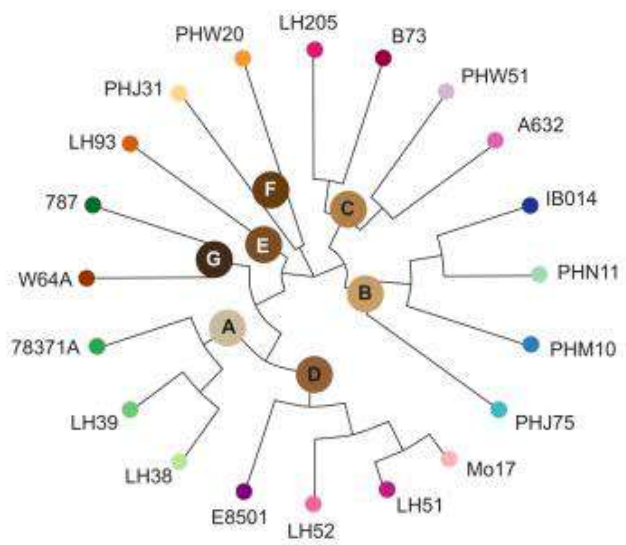

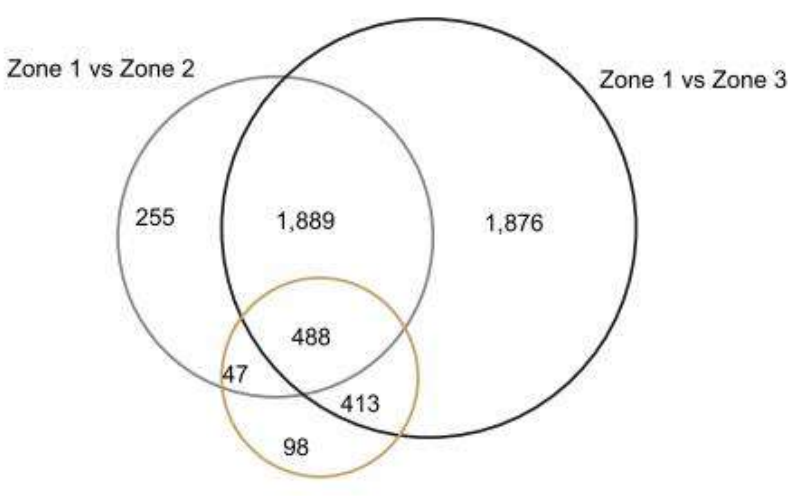

Zone 2 vs Zone 3

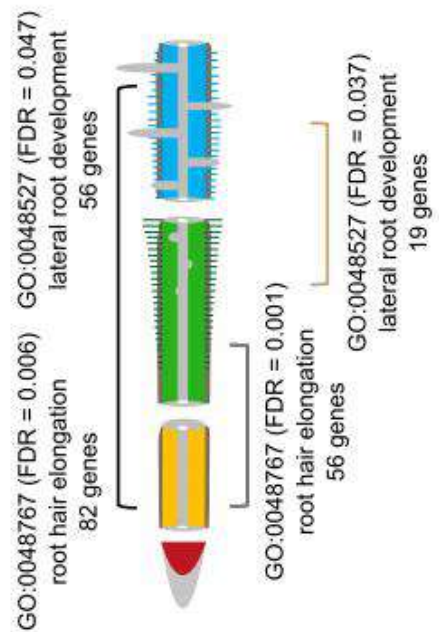

\section{Supplementary Figure 1. Transcriptome signature synchronizes with developmental changes along}

the root zones of maize genotypes. a, Representative 4-week-old B73 root system from the field after removing the loosely attached soil particles by shaking. The localization of $2^{\text {nd }}$ whorl of crown root with rhizosphere is indicated by a white arrow. $\mathbf{b}$, Neighbour-joining phylogenetic tree showing relationships of 20 diverse maize inbred lines. This tree was produced based on Illumina MaizeSNP50 data. Lines were coloured and clustered into 7 groups (A-G) according to their phylogenetic distances. c, Venn diagram showing specificity of differentially expressed genes between each pair of zones. Separation strategy of developmental zones refers to the Figure 1a. Differentially expressed genes are declared as the fold change $>2$ or $<-2$ and FDR (false discovery rate) corrected $p<0.05$. Functional annotations (biological process) of the differentially expressed genes for each pair of zones are classified by agriGO (v2) using gene ontology $(\mathrm{GO})$ with statistical significance calculated by Fisher's exact test with Yekutieli adjustment (FDR corrected $p<0.05$ ). The whole list of differentially expressed genes is provided in the Supplementary Dataset 1. 

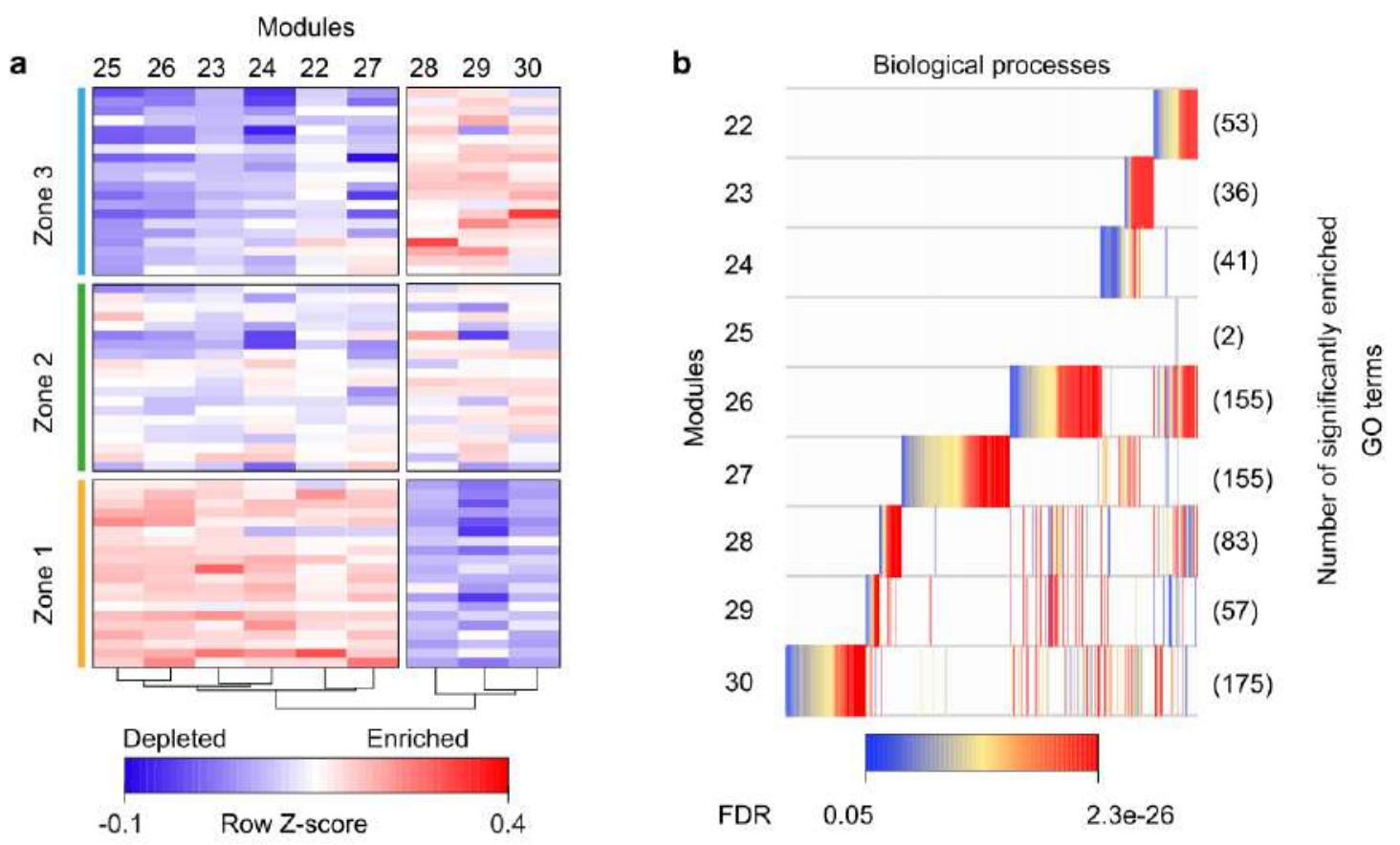

Supplementary Figure 2. Dynamic patterns and functional associations of gene co-expression modules along root developmental zones. a, Heat map illustrating six depleted and three enriched modules from the younger region to the older region along root developmental zones. $\mathbf{b}$, Functional annotations (biological process) of genes from each module by agriGO (v2.0). The number of significant GO terms for each module is given in brackets. Statistical significance of GO enrichment for each module is calculated by Fisher's exact test with Yekutieli adjustment (FDR corrected $p<0.05$ ). 

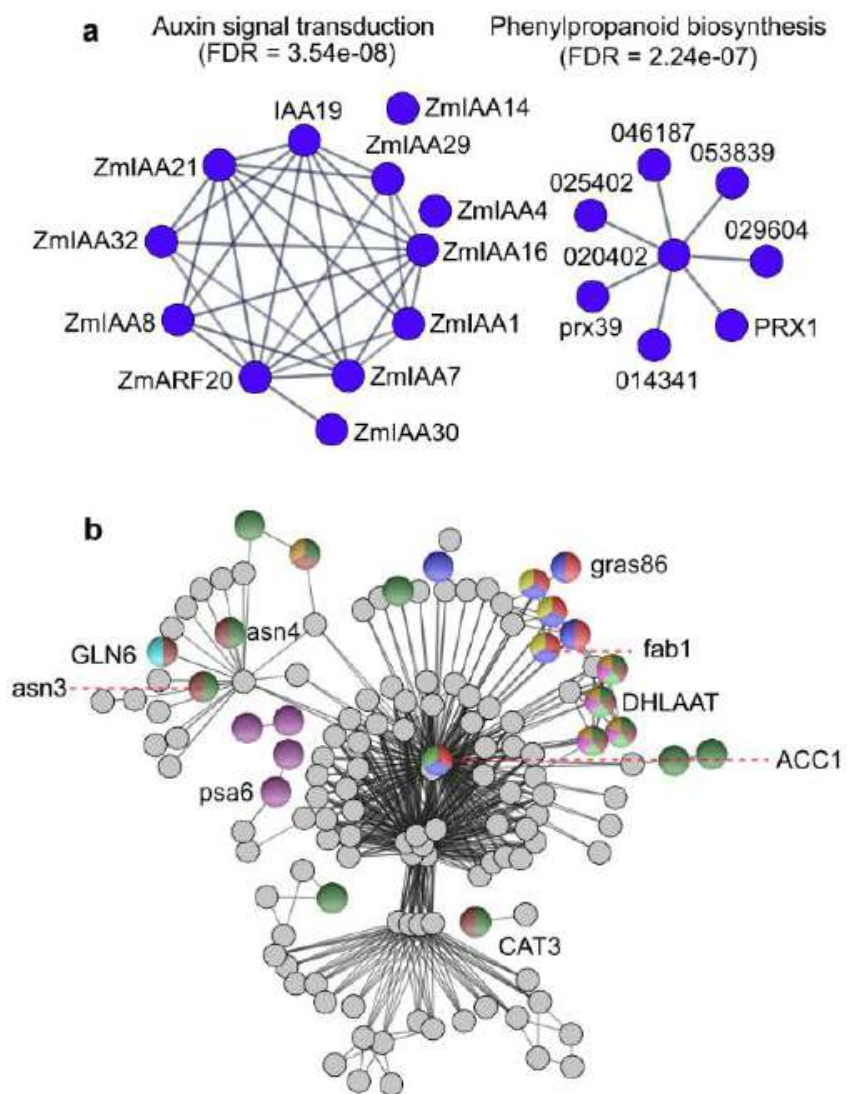

$\begin{array}{ll}\text { KEGG pathways } & \text { FDR } \\ \text { Fatty acid biosynthesis (6) } & 2.04 \mathrm{e}-05 \\ \text { Fatty acid metabolism (7) } & 7.18 \mathrm{e}-05 \\ \text { Biosynthesis of secondary } & 0.00262 \\ \text { metabolites (19) } & \\ \text { Microbial metabolism in } & 0.00262 \\ \text { diverse environments (10) } & \\ \text { Biotin metabolism (3) } & 0.00785 \\ \text { Pyruvate metabolism (5) } & 0.00905 \\ \text { Citrate cycle (TCA cycle) (4) } & 0.0199 \\ \text { Nitrogen metabolism (3) } & 0.0227 \\ \text { Photosynthesis (4) } & 0.0287 \\ \text { Glycolysis/Gluconeogenesis (5) } & 0.0411\end{array}$

Supplementary Figure 3. Inference of protein-protein network interactions within growth (a) and defence-related (b) modules. Genes from each module were imported into the STRING database (v10.5) to query the experimentally determined interactions among genes at the high confidence 0.7 level. Those interacting genes were further functionally enriched into KEGG pathways. The significances of both networks are defined as protein-protein interaction (PPI) $<1.0 \mathrm{e}^{-16}$ and enriched KEGG pathways are controlled by the FDR corrected $p<0.05$ within the STRING database. ACC, acetyl-CoA carboxylase; ARF, auxin response factor; asn, asparagine synthetase; CAT, catalase; DHLAAT, dihydrolipoamide acetyl transferase; fab, fatty acid biosynthesis; GLN, gluthatione synthetase; IAA, indole-3-acetic acid; psa, photosystem I subunit; PRX, Peroxiredoxin. The whole list of genes enriched in networks is provided in Supplementary Datasets 5 and 6. 




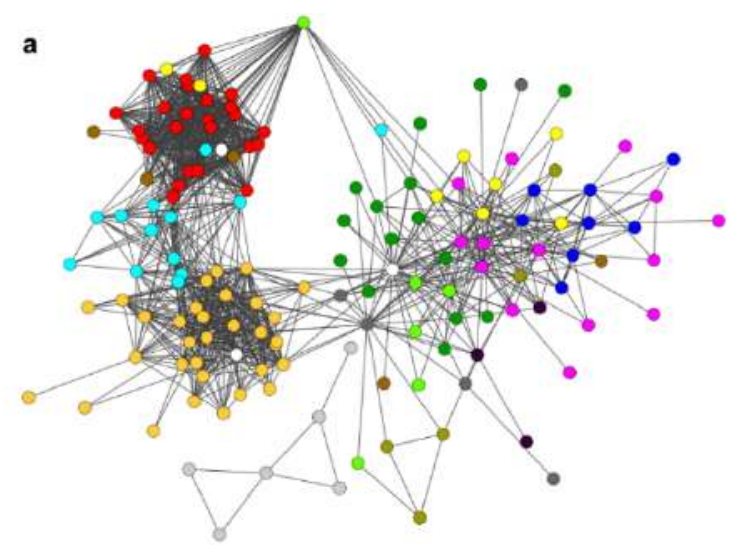

b
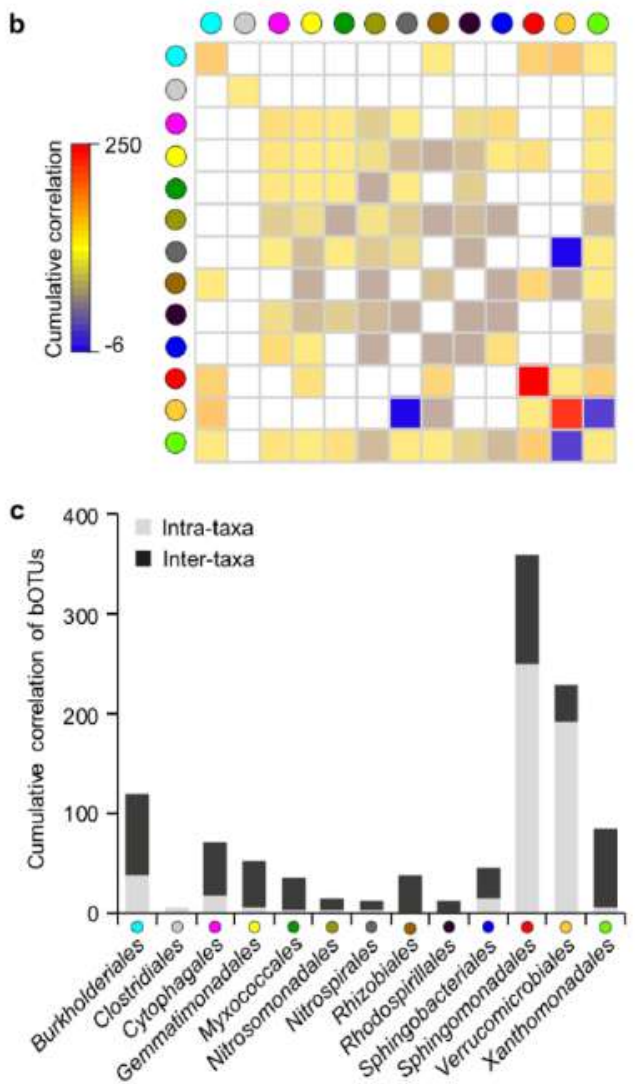

Supplementary Figure 6. Microbial network of maize rhizosphere microbiota. a, Correlation-based network of genotype and zone-associated microbial OTUs detected in the rhizosphere. Each node corresponds to an OTU, and edges between nodes correspond correlations inferred from OTU abundance profiles using the SparCC method (two-tailed pseudo $p<0.05$, correlation values $<-0.6$ or $>0.6$ ). The analysis was conducted with default parameters and 100 bootstrap samples were used to infer pseudo $p$ values. OTUs belonging to different bacterial taxa have distinct colour codes in the rhizosphere. $\mathbf{b}$, Cumulative correlation scores measured in the bacterial network among different bacterial orders. c, The proportion of edges showing inter-taxa (black) and intra-taxa (grey) correlations in the bacterial rhizosphere network. 


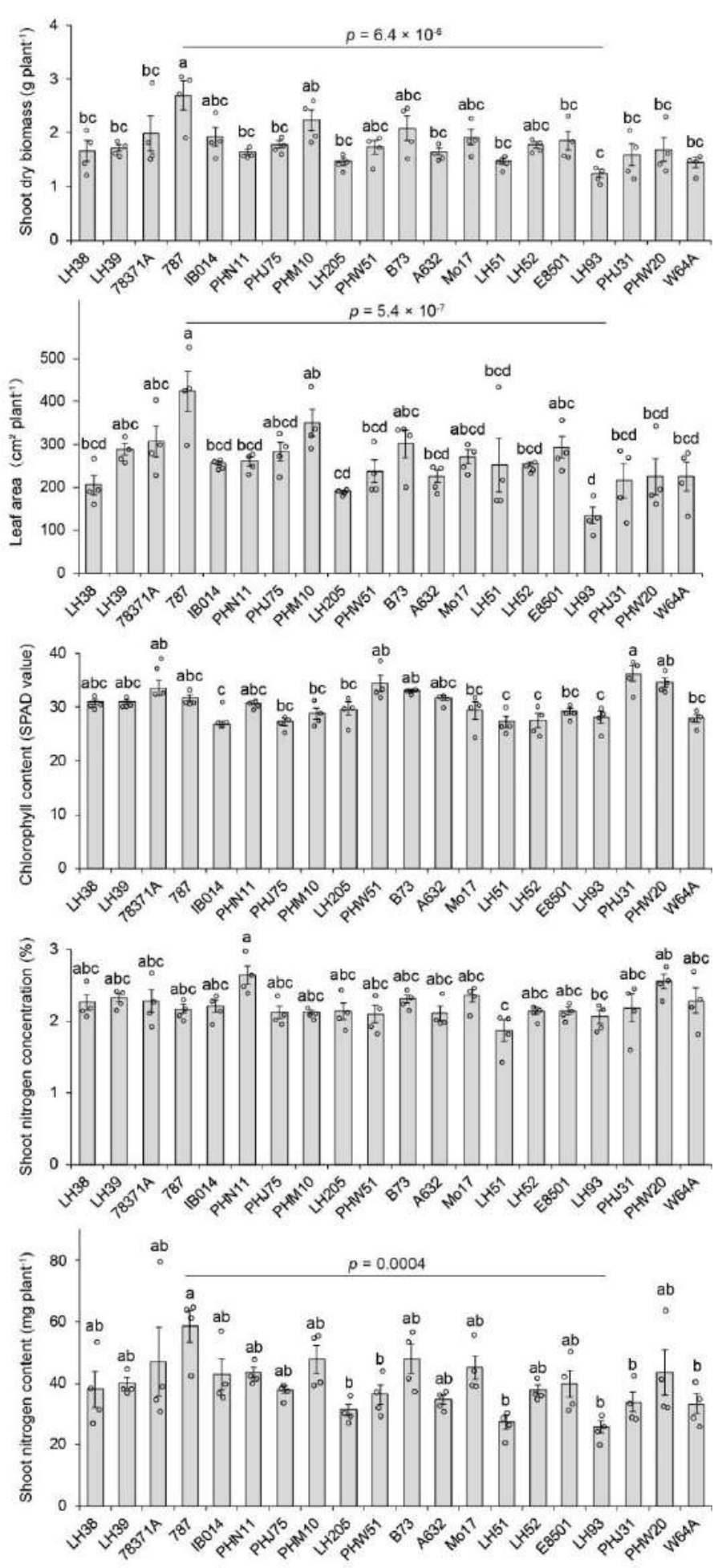

Supplementary Figure 7. Phenotypic differences of 20 maize inbred lines grown in the field. Nondestructive measurement of chlorophyll content is evaluated by SPAD (Soil Plant Analysis Development) value determined as average of 30 measurements in the middle third of the last expanded leaf. Different letters denote significance at $p<0.05$ (ANOVA, Tukey HSD). Significant differences between 787 and LH93 were indicated by exact $p$ values. $n=4$ biologically independent samples. Data are mean \pm s.e.m. 
a

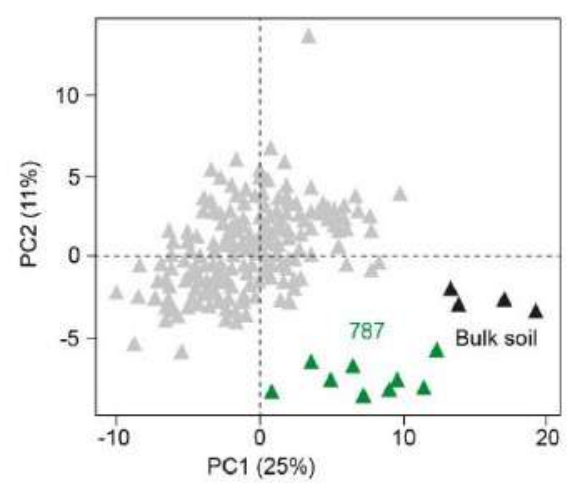

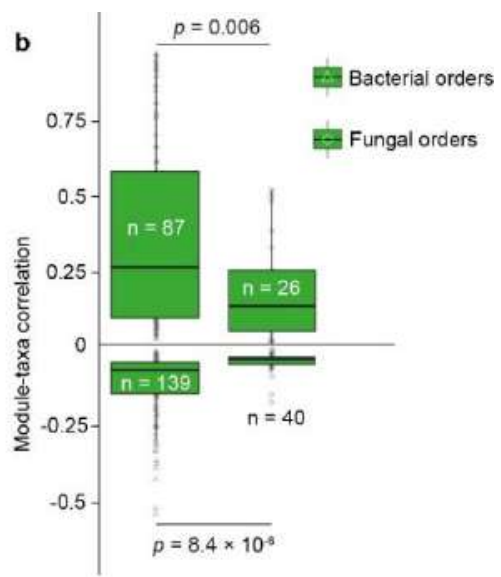

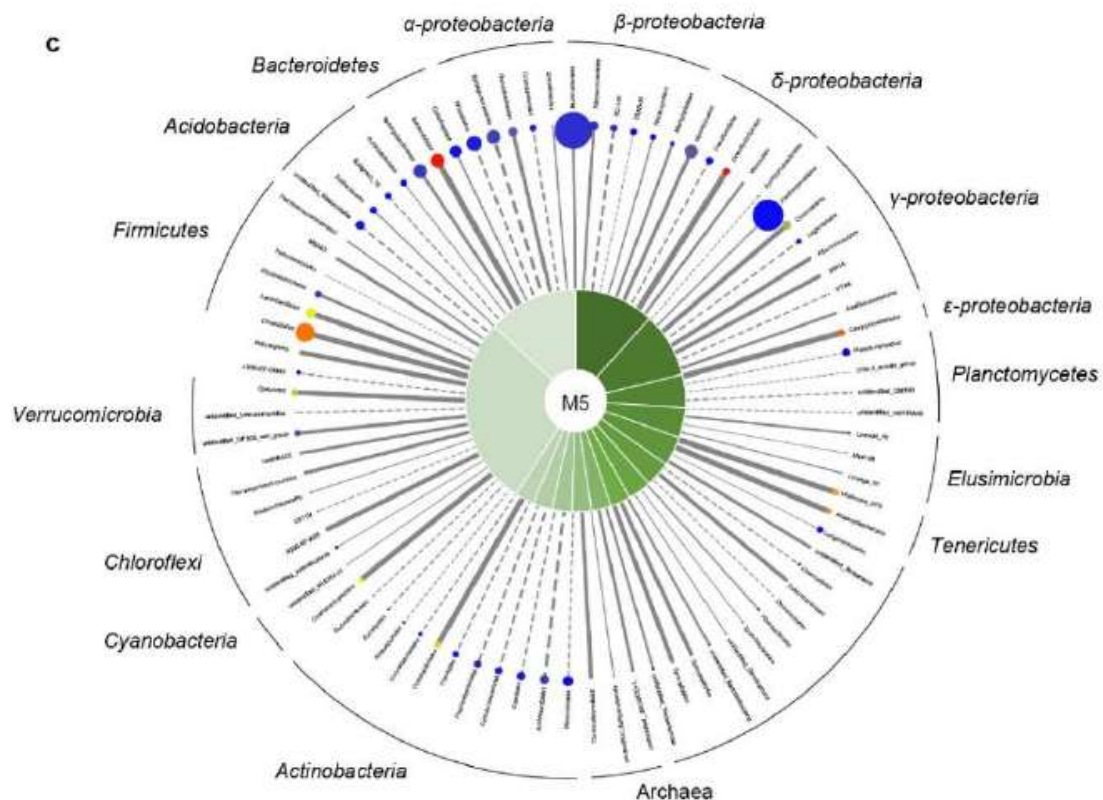

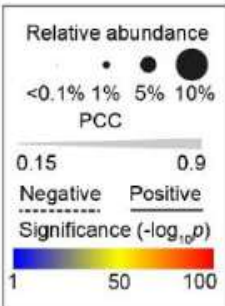

- Burkholderiales

- Xanthomonadales

- Clostridiales

- Verrucomicrobiales

- Pseudomonadales

- Rhodospirillales

- Rhizobiales

- Sphingobacteriales

- Enterobacteriales

- Bacteroidales

- Sphingomonadaies

- Bacillales

$=$ Myxococcales

$<3 \%$

Others

Supplementary Figure 8. Specificity of maize genotype 787 rhizosphere microbiota. a, PCA plot showing the dissimilarity of bacterial community of the 787 rhizosphere (green triangles) in comparison to the other 19 genotypes (grey triangles) and bulk soil (black triangles). b. Functional associations between 787-enriched module and microbial orders. $p$ values, which were calculated using two-tailed Wilcoxon rank sum test, are indicating significance. The boxes span from the first to the third quartiles, the centre lines represent the median values and the whiskers show the data that lie within the 1.5 interquartile range of the lower and upper quartiles. The data points at the ends of the whiskers represent the outliers. c, Composition of the 787 bacterial community and its module correlation with different bacterial orders. Node size indicates the relative abundance. Edge thickness indicates the strength of Pearson's correlation coefficient. Colour scale indicates the significant levels of correlation. The exact FDR-corrected significant $p$ values are shown in the Source Data. 

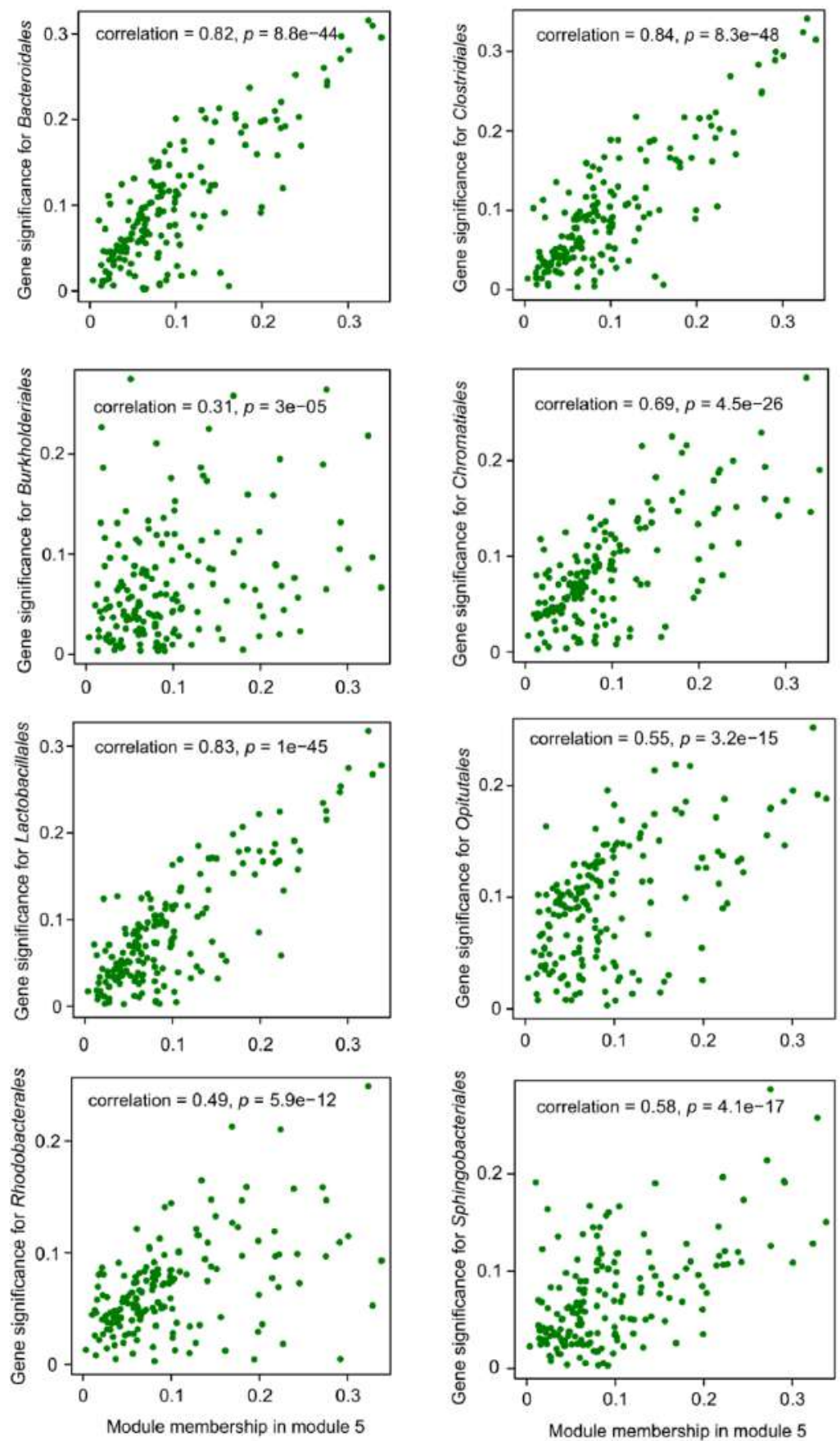

Supplementary Figure 9. Scatterplots of correlations between gene significance and module membership for 787-enriched module 5 related to specific bacterial orders. Correlation-based analysis inferred by WGCNA between 787-specific module and the bacterial taxa the order level. Pearson's correlation is calculated by relating the eigengene of each module with the relative abundance of each taxon using WGCNA "trait" function. Gene significances between module membership are controlled by FDR corrected $p$ values. 

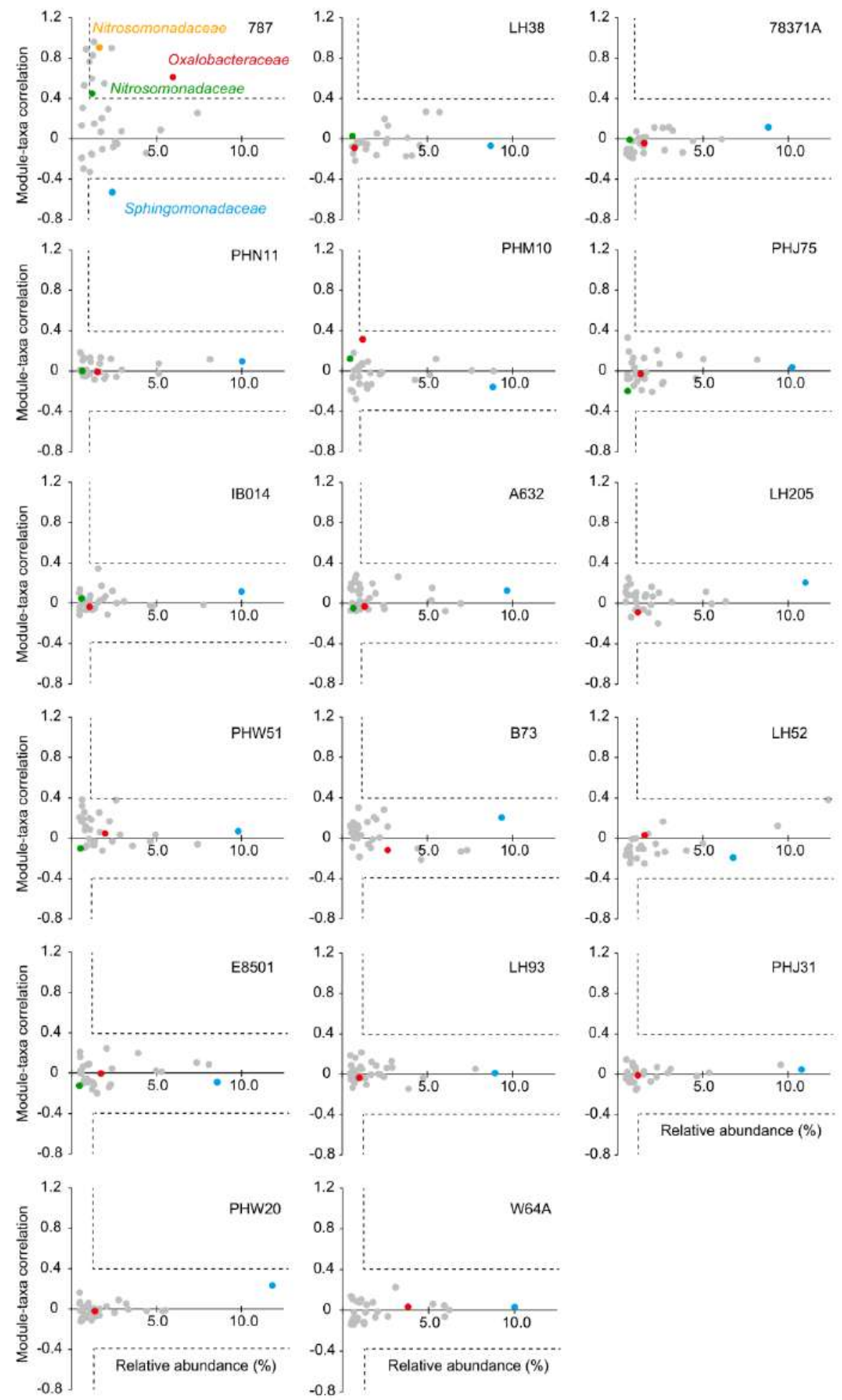

Supplementary Figure 10. Family-level module-taxa correlation highlights unique feature of 787 interaction with bacteria. These scatter plots show the Pearson's correlations between bacterial families with $\geq 1 \%$ relative abundance and eigengenes of genotype-specific modules (Figure 2a) identified by WGCNA. Oxalobacteraceae and Sphingomonadaceae are highlighted in red and blue for all maize inbred lines, respectively. 



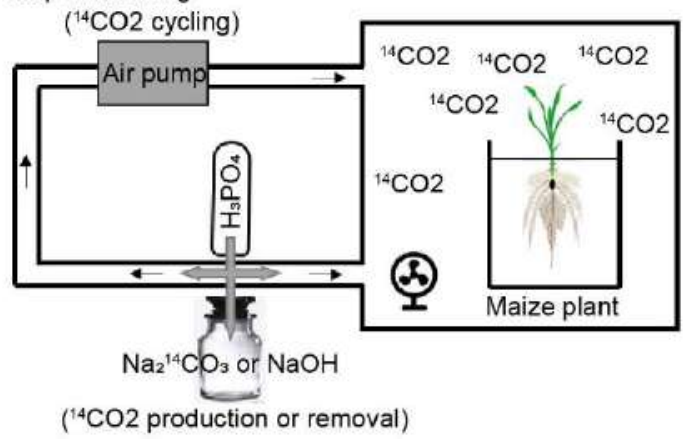

Step 2: imaging
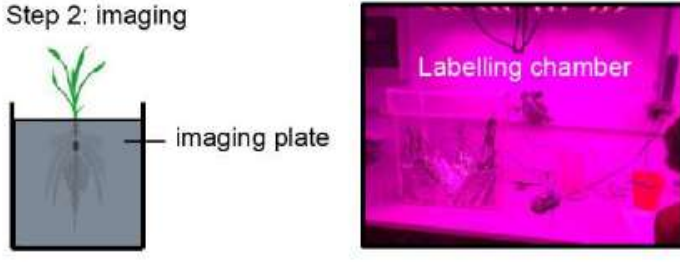

C

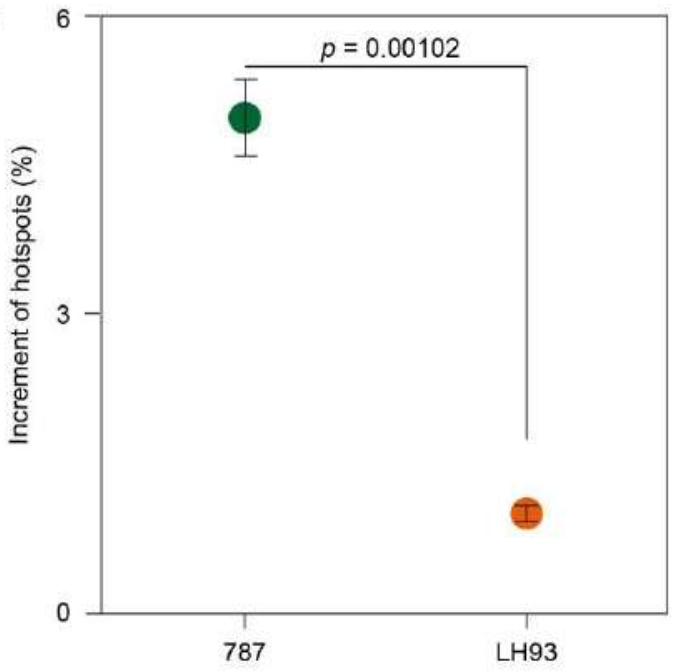

b

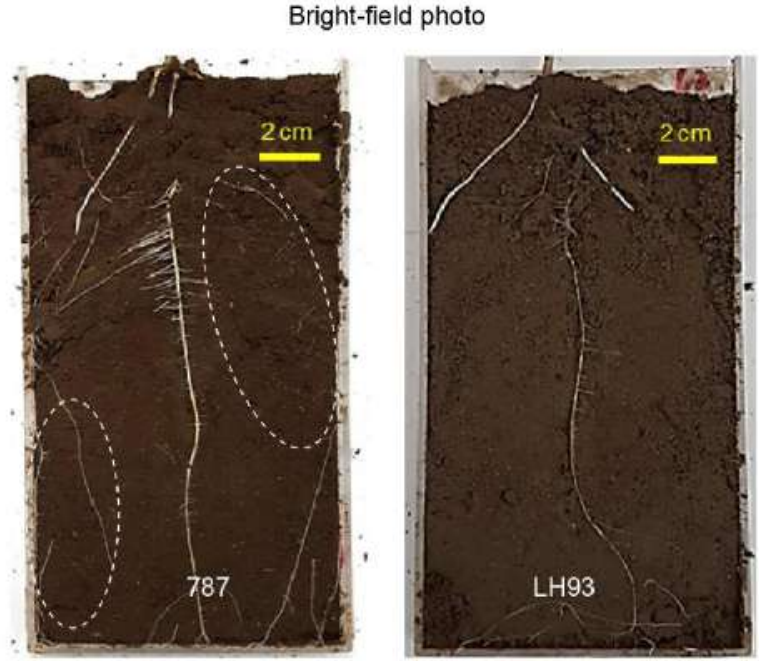

${ }^{14} \mathrm{C}$ fluorescence imaging
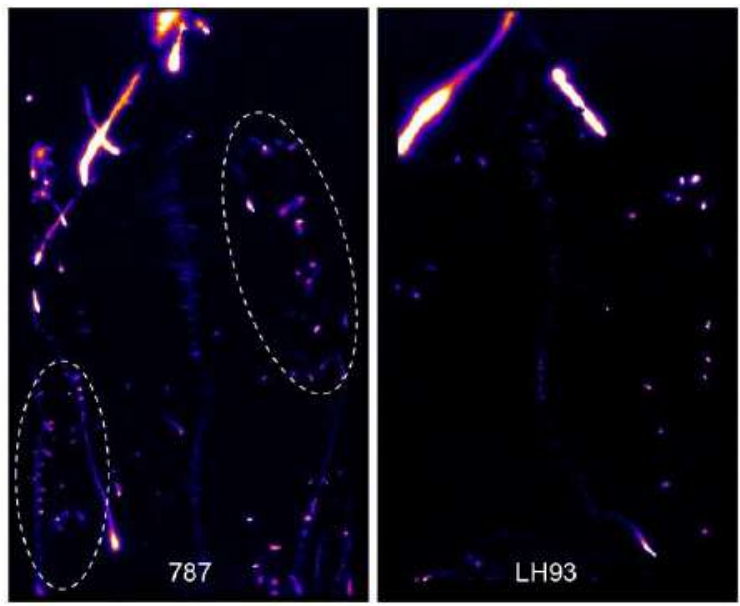

Photo stimulated luminescence

Supplementary Figure 12. Distribution of root exudates in the rhizosphere among root types between the 787 and LH93. a, Schematic illustration of ${ }^{14} \mathrm{C}$ pulse labelling and imaging for maize roots grown in the rhizobox. b. Comparison of root carbon exudation between 787 and LH93 by ${ }^{14} \mathrm{C}$ pulse labelling and imaging. Hot spots of exudation from the lateral roots are highlighted by the dotted circles. c, Quantification results are shown as increment of the hotspots (\%). Data are presented as mean values \pm s.e.m and $n=3$ biologically independent samples. Significance was calculated using two-tailed unpaired ttest and indicated with exact value. 


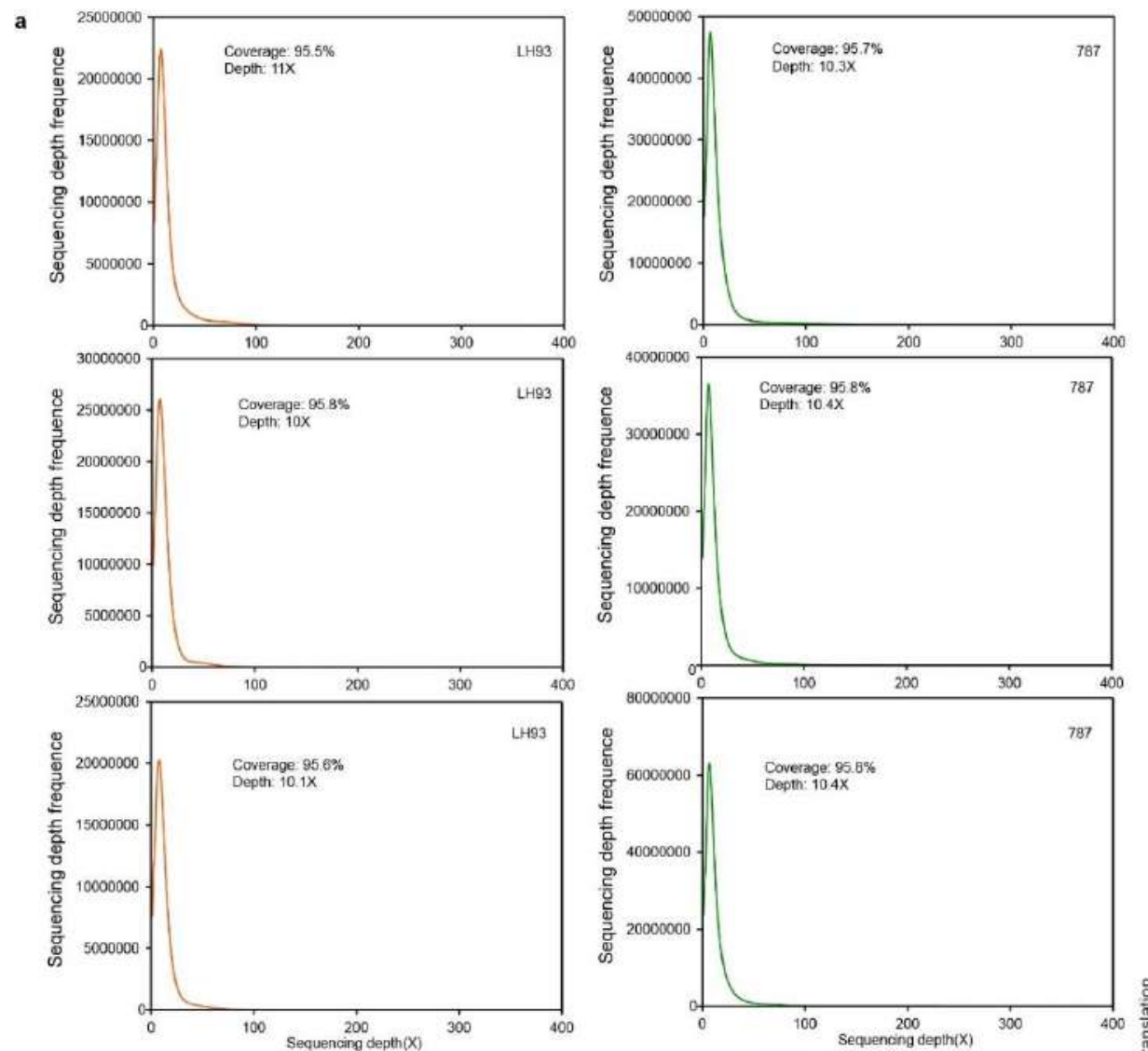

b
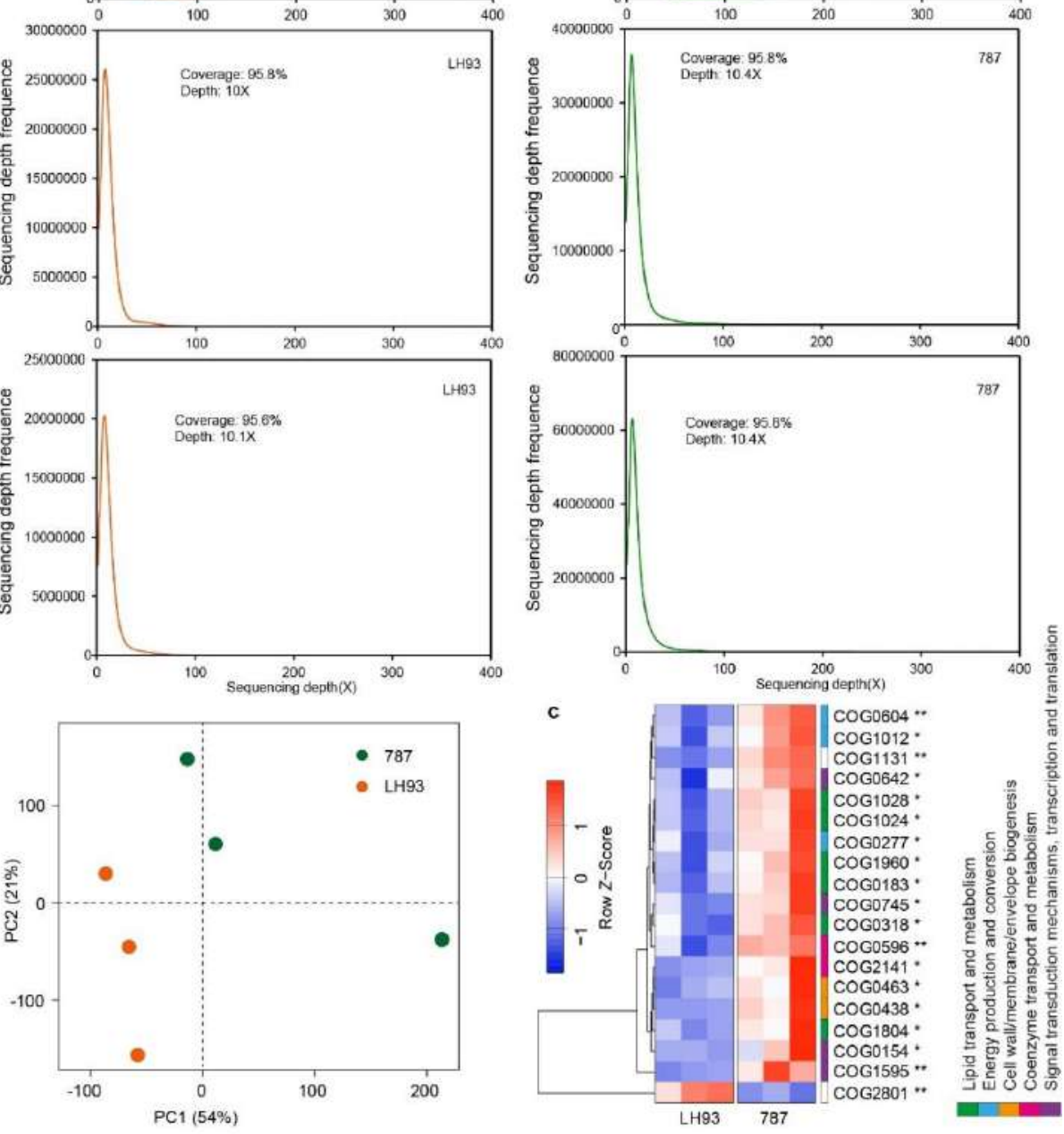

Supplementary Figure 14. Functional differences between the 787 and LH93 microbiomes. a, Coverage and sequencing depth of shotgun metagenomics sequencing of 787 and LH93 rhizosphere microbiomes. b, PCA plot indicating distinct functional gene profiles associated with 787 and LH93 rhizosphere metagenome. c, Enrichment of functional categories of COG (Clusters of Orthologous Groups) families for the rhizosphere metagenome between 787 and LH93. Mean proportion of functional genes (\%) normalized by Row-Z score and asterisks indicate significant differences between 787 and LH93 rhizosphere microbiome with two-tailed unpaired t-test (FDR adjusted ${ }^{*} p<0.05,{ }^{* *} p<0.01$ ). $n=3$ biologically independent samples. 

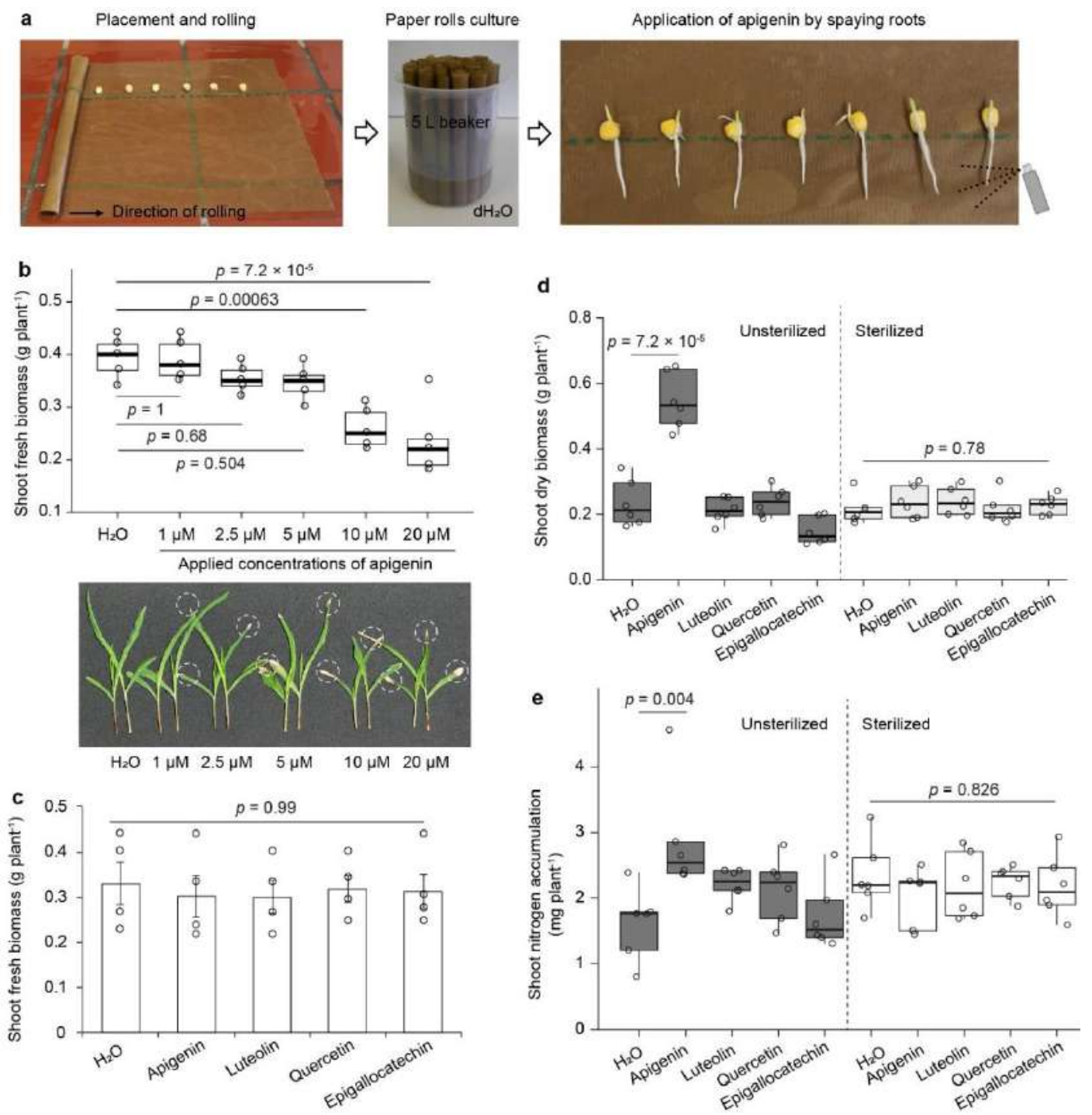

Supplementary Figure 15. Effects of exogenous flavonoids on maize growth in the paper roll system and soil pots. a, Illustration of the paper roll system used in this study and workflow for flavonoid application. b. Effects of different concentrations of apigenin on maize shoot growth in the paper roll system. $n=5$ biologically independent samples. The top and bottom of boxes indicate interquartile range $(75 \%$ to $25 \%$ of the data) and the median values are shown as horizontal bars within boxes. Leaf damages caused by overapplied concentrations of apigenin are highlighted by dotted circles. c, Effects of different types of flavonoids $(1 \mu \mathrm{M})$ on maize shoot growth in the paper roll system. $n=4$ biologically independent samples. Data are mean \pm s.e.m. Effects of exogenously applied flavonoid types on C2-idf growth (d) and nitrogen accumulation (e) in nitrogen-poor soil with and without prior sterilization. For $\mathbf{d}$ and $\mathbf{e}, n=6$ biologically independent samples. For $\mathbf{b}, \mathbf{d}$ and $\mathbf{e}$, the boxes span from the first to the third quartiles, the centre lines represent the median values and the whiskers show the data that lie within the 1.5 interquartile range of the lower and upper quartiles. The data points at the ends of the whiskers represent the outliers. For $\mathbf{b}, \mathbf{c}$, $\mathbf{d}$ and $\mathbf{e}$, significances were indicated among different groups by exact $p$ values (ANOVA, Tukey HSD). 



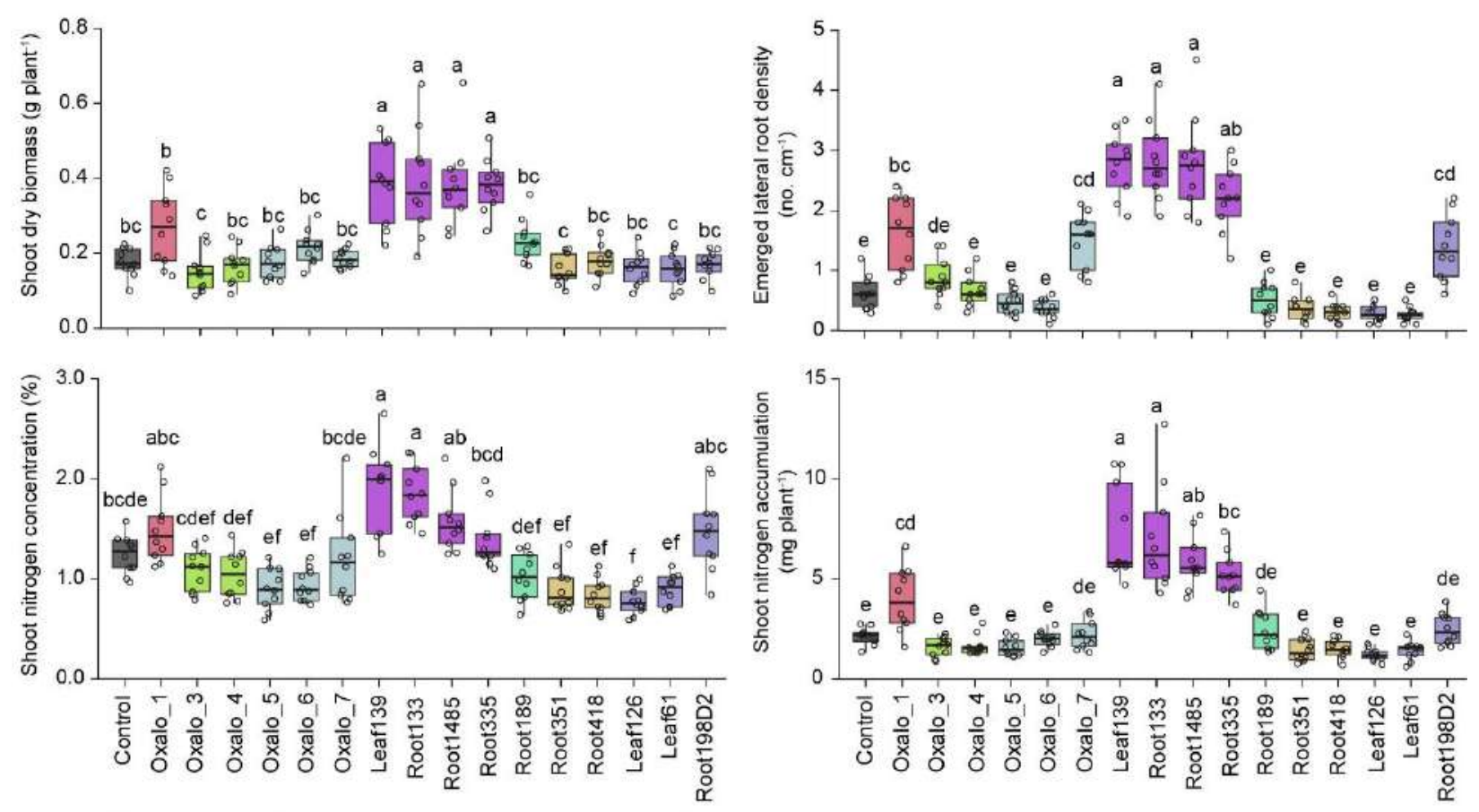

Duganella

Massitia

\section{Supplementary Figure 18. Effects of Oxalobacteraceae strains inoculation on growth promotion and} nitrogen uptake in Irt1 mutant in nitrogen-poor soil. Different letters indicate significantly different groups ( $p<0.05$, ANOVA, Tukey HSD). $n=10$ biologically independent samples over two independent inoculation experiments. The boxes span from the first to the third quartiles, the centre lines represent the median values and the whiskers show the data that lie within the 1.5 interquartile range of the lower and upper quartiles. The data points at the ends of the whiskers represent the outliers. 


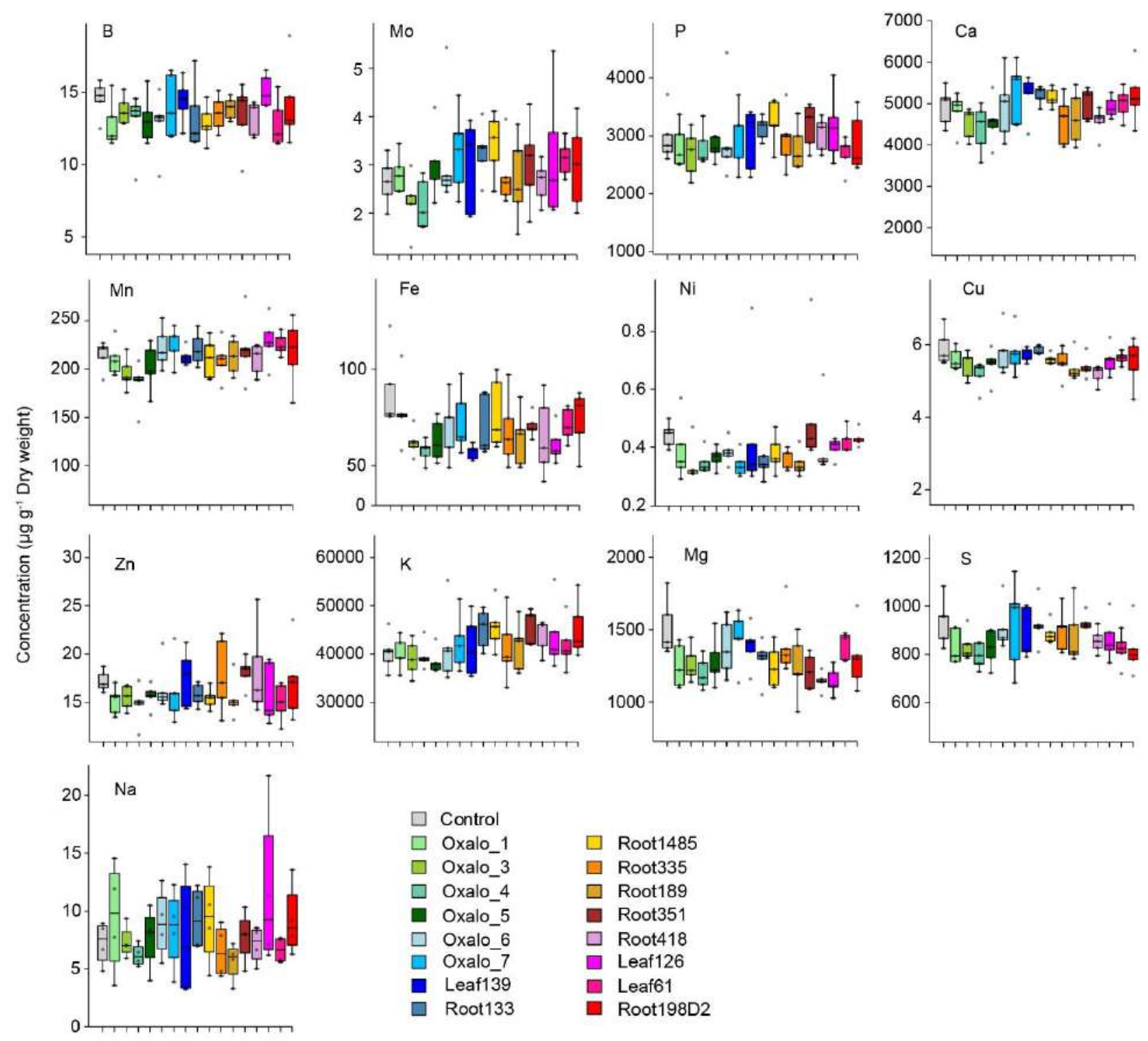

Supplementary Figure 19. Effects of Oxalobacteraceae strains inoculation on the concentrations of 13 mineral nutrients in Irt1 mutant in nitrogen-poor soil. No significant differences were detected at $p<0.05$ (ANOVA, Tukey HSD). $n=5$ biologically independent samples. The boxes span from the first to the third quartiles, the centre lines represent the median values and the whiskers show the data that lie within the 1.5 interquartile range of the lower and upper quartiles. The data points at the ends of the whiskers represent the outliers. 



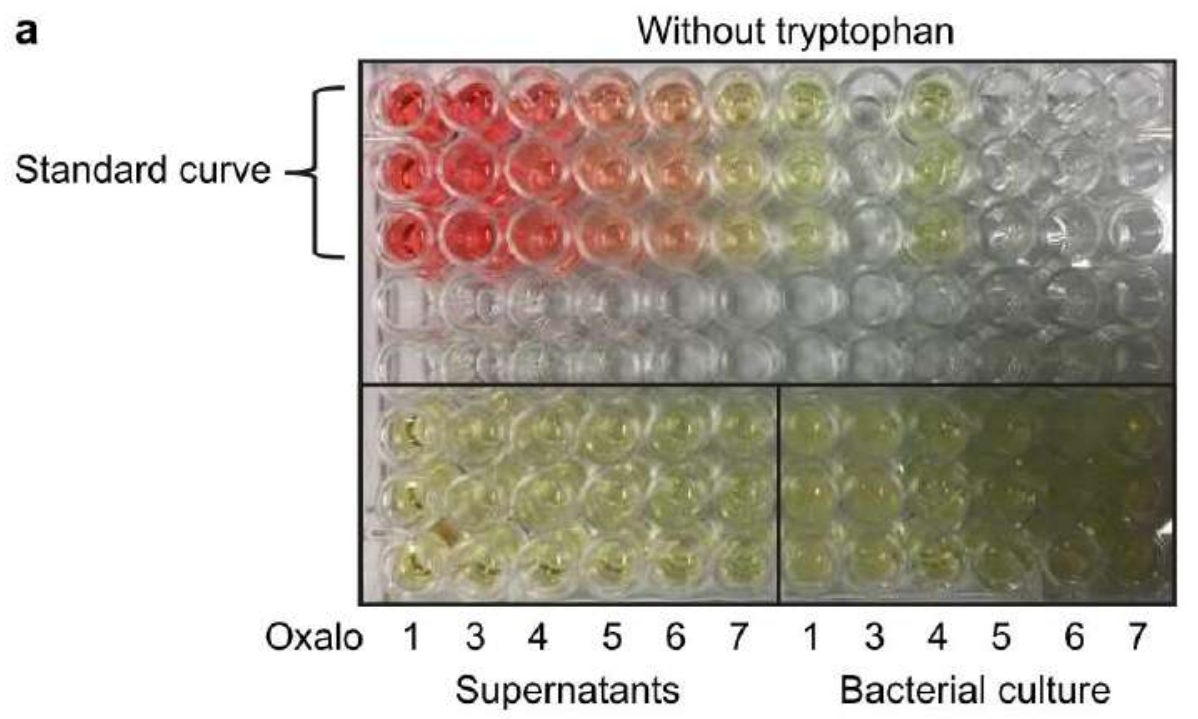

b

With tryptophan

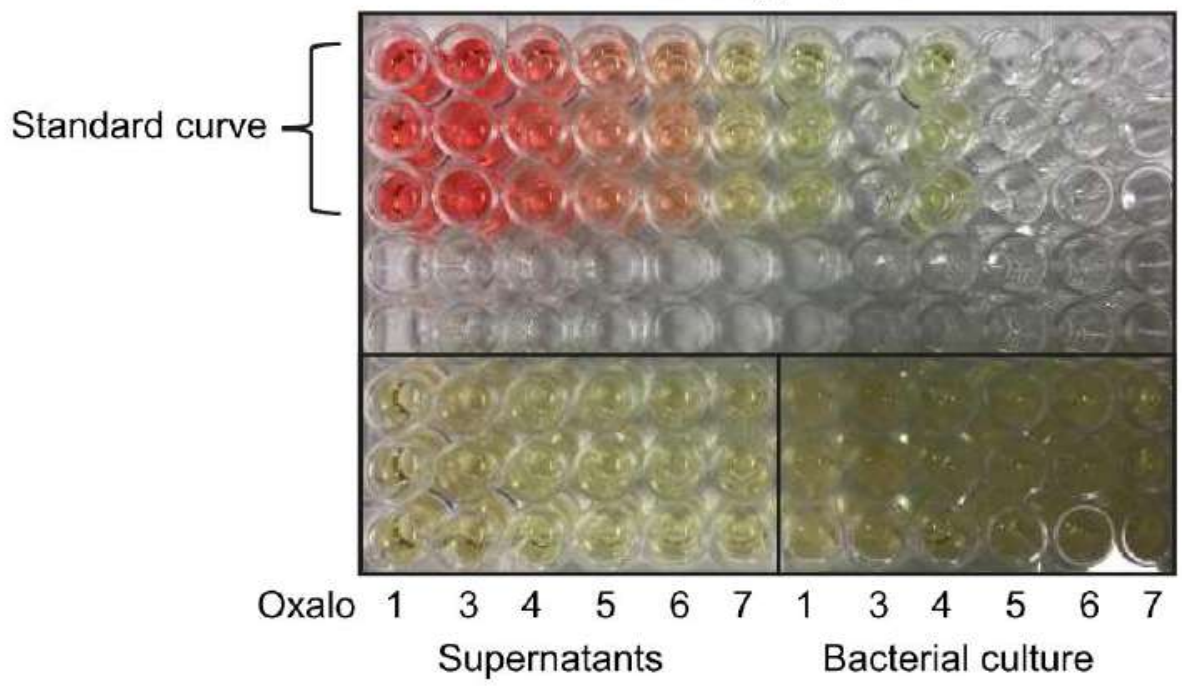

Supplementary Figure 21. Determination of indolic compounds produced by Oxalobacteraceae strains based on supernatants and bacterial cultures. a, Without tryptophan. b, With tryptophan. Colorimetric analysis by the reagent of Salkowski of stationary phase cultures indicated that Oxalobacteraceae isolates do not produce auxin in the presence and absence of tryptophan. 


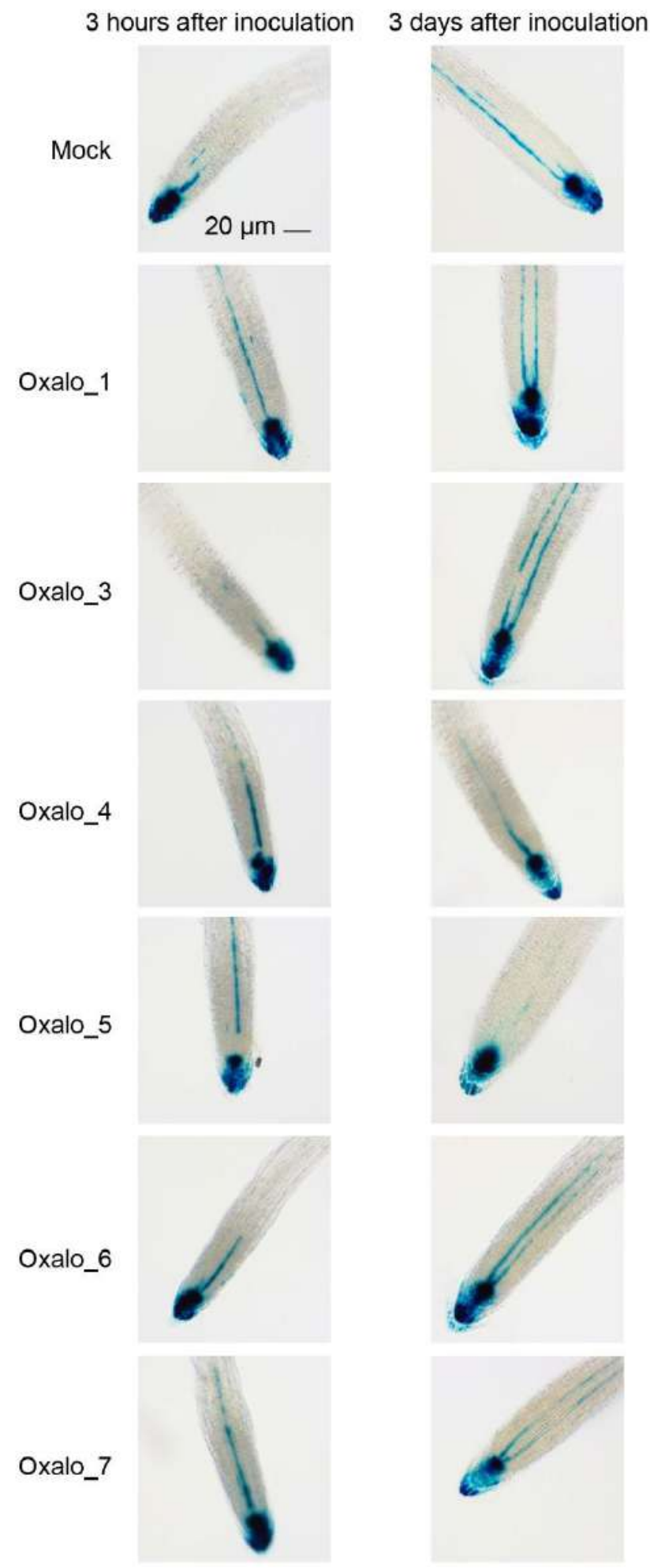

Supplementary Figure 22. Auxin induction tests in Arabidopsis by Oxalobacteraceae strains. Expression of DR5::GUS signal along the longitudinal root of Arabidopsis after $3 \mathrm{~h}$ and $3 \mathrm{~d}$ inoculation with different Oxalobacteraceae strains. 


\section{a}
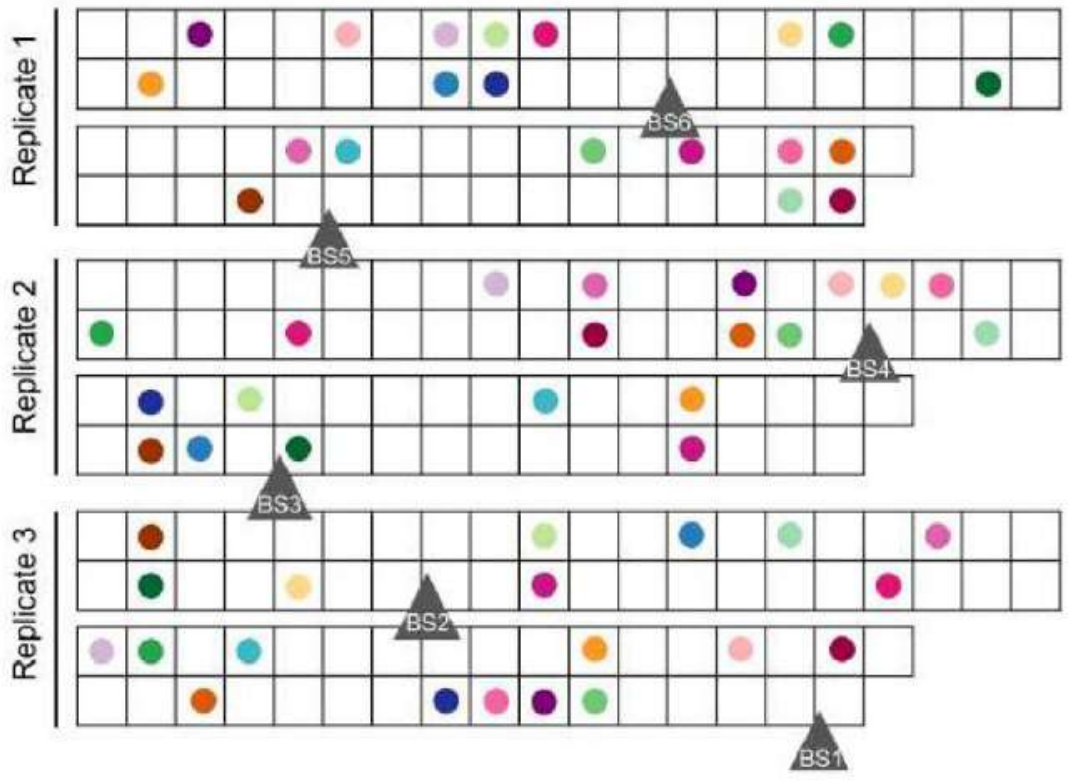

b

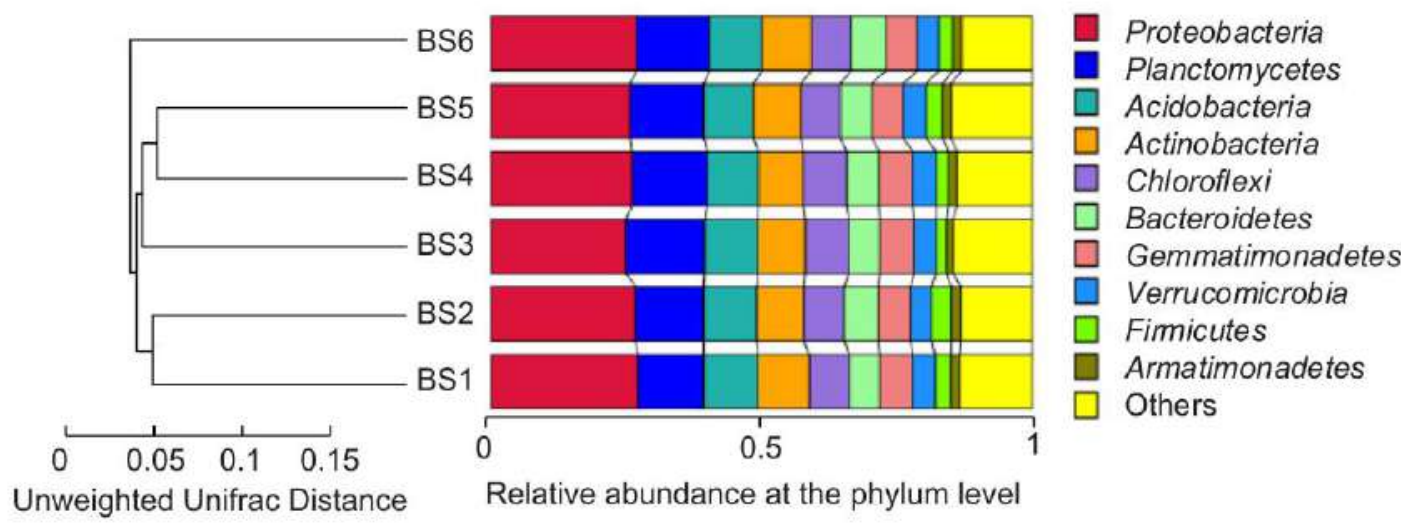

Supplementary Figure 23. The experimental design, germplasm used and soil homogeneity in this field study. a, Complete random design of the field experiment and sampling sites of the maize inbred lines. In total, 72 US public inbred lines from the Ames population were sown in the field and the coloured 20 healthy grown lines indicate their distributions for three biological replicates in the field. Six basic soil samples with the triangle symbols were collected for preliminary test of bacterial diversity before sowing. Colour dots correspond to different maize genotypes in Supplementary Fig. 1b. b, Homogeneous distribution of bulk soil microbiome from the basic soil samples. $\beta$-diversity heat map was compared for six basic soil samples at the phylum level using unweighted unifrac distance. BS, basic soil. 


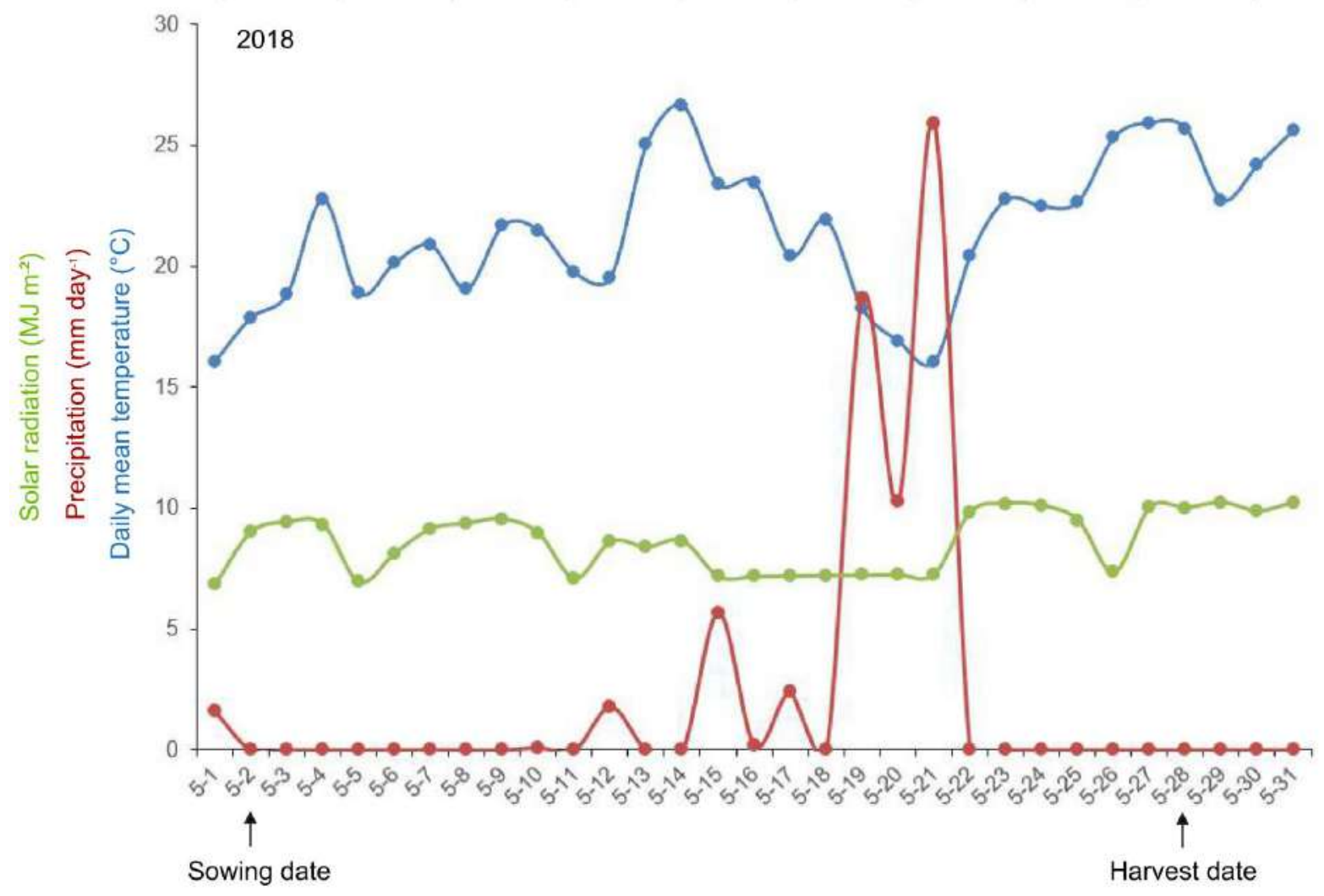

Supplementary Figure 24. Meteorological data tracked during the 4-week growth period in the field. Daily mean temperature, precipitation per day and solar radiation were colour-coded accordingly. The sowing and harvest dates were indicated by black arrows. 

Supplementary Table 3. Interactions of the gene and OTU according to significant factors. The prefiltered genes were used as input to identify differentially expressed genes for each OTU (bacterial and fungal) by using the R package DESeq2 with the model gene expression OTU abundance. OTU abundance was taken as a trait to explain gene expression. Significant genes were determined with corresponding bOTUs and fOTUs with FDR and $\log _{2}$ fold change for $\alpha$ levels of 0.001 . The same analysis was performed as above but explaining bOTU abundance with fOTU abundance and vice versa.

\begin{tabular}{ccccccccccc}
\hline & $\mathbf{x}$ & Total $\mathbf{x}$ & $\begin{array}{c}\text { Unique } \\
\mathbf{x}\end{array}$ & $\begin{array}{c}\% \\
\text { unique }\end{array}$ & $\mathbf{y}$ & $\begin{array}{c}\text { Total } \\
\mathbf{y}\end{array}$ & $\begin{array}{c}\text { sign. } \\
\mathbf{y} \mathbf{0} \\
\mathbf{x}\end{array}$ & $\begin{array}{c}\% \\
\text { sign. } \\
\mathbf{y}\end{array}$ & $\begin{array}{c}\text { Total } \mathbf{x} \\
\begin{array}{c}\mathbf{x} \\
\mathbf{p e r} \\
\mathbf{y}\end{array}\end{array}$ \\
\hline $\begin{array}{c}\text { Genes } \sim \text { bOTU- } \\
1 \%\end{array}$ & Genes & 27092 & 12907 & 0.48 & bOTU & 736 & 720 & 0.98 & 797361 & 1107 \\
\hline $\begin{array}{c}\text { Genes bOTU- } \\
0.1 \%\end{array}$ & Genes & 27092 & 7342 & 0.27 & bOTU & 736 & 700 & 0.95 & 284729 & 407 \\
\hline $\begin{array}{c}\text { Genes fOTU- } \\
1 \%\end{array}$ & Genes & 27092 & 4043 & 0.15 & fOTU & 18 & 18 & 1 & 8248 & 458 \\
\hline $\begin{array}{c}\text { Genes fOTU- } \\
0.1 \%\end{array}$ & Genes & 27092 & 1553 & 0.06 & fOTU & 18 & 17 & 0.94 & 2761 & 162 \\
\hline $\begin{array}{c}\text { bOTU fOTU-1\% } \\
\text { bOTU fOTU- } \\
0.1 \%\end{array}$ & bOTU & 736 & 47 & 0.06 & fOTU & 18 & 11 & 0.61 & 75 & 7 \\
\hline fOTU bOTU-1\% & fOTU & 18 & 1 & 0.06 & bOTU & 736 & 3 & 0 & 3 & 1 \\
\hline $\begin{array}{c}\text { fOTU bOTU- } \\
0.1 \%\end{array}$ & fOTU & 18 & 0 & 0 & bOTU & 736 & 0 & 0 & 0 & 0 \\
\hline
\end{tabular}

\begin{tabular}{ccccccc}
\hline Overlap & $\begin{array}{c}\text { Genes } \\
\text { OOTUs }\end{array}$ & Total x & Overlap & $\begin{array}{c}\% \text { of } \\
\text { total }\end{array}$ & Unique & $\begin{array}{c}\% \text { of } \\
\text { total }\end{array}$ \\
\hline $\begin{array}{c}\text { Genes bOTU- } \\
1 \%\end{array}$ & 12907 & 13402 & 3549 & 0.26 & 9359 & 0.7 \\
\hline $\begin{array}{c}\text { Genes fOTU- } \\
1 \%\end{array}$ & 4043 & 13402 & 3549 & 0.26 & 494 & 0.04 \\
\hline $\begin{array}{c}\text { Genes bOTU- } \\
0.1 \%\end{array}$ & 7342 & 7534 & 1363 & 0.18 & 5980 & 0.79 \\
\hline $\begin{array}{c}\text { Genes fOTU- } \\
0.1 \%\end{array}$ & 1553 & 7534 & 1363 & 0.18 & 191 & 0.03 \\
\hline
\end{tabular}

Supplementary Table 4. Basic soil chemical properties before fertilization in the field.

\begin{tabular}{cccccc}
\hline Parameters & $\mathrm{pH}$ & $\begin{array}{c}\text { Organic matter content } \\
(\mathrm{g} / \mathrm{kg})\end{array}$ & $\begin{array}{c}\text { Total nitrogen } \\
(\mathrm{g} / \mathrm{kg})\end{array}$ & $\begin{array}{c}\text { Available } \\
\text { phosphorus } \\
(\mathrm{mg} / \mathrm{kg})\end{array}$ & $\begin{array}{c}\text { Exchangeable } \\
\text { potassium } \\
(\mathrm{mg} / \mathrm{kg})\end{array}$ \\
\hline Values & 8.5 & 14.1 & 0.9 & 6.7 & 100.4 \\
\hline
\end{tabular}

